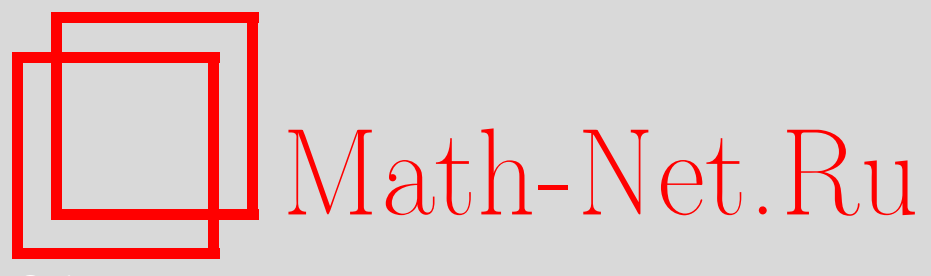

А. С. Демидов, Функционально-геометрический метод решения задач со свободной границей для гармонических функций, УМH, 2010, том 65, выпуск 1, 3-96

DOI: https://doi.org/10.4213/rm9341

Использование Общероссийского математического портала Math-Net.Ru подразумевает, что вы прочитали и согласны с пользовательским соглашением http://www . mathnet.ru/rus/agreement

Параметры загрузки:

IP : 54.172 .240 .79

26 апреля 2023 г., 15:37:31

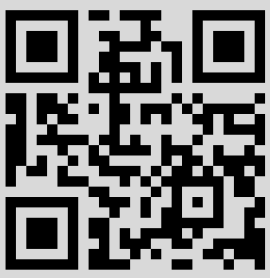



ния одного метода, позволяющего решать некоторые задачи о потенциальных функциях, ранее представлявшие затруднения."

Г. Гельмгольи, [1]

\title{
Функционально-геометрический метод решения задач со свободной границей для гармонических функций
}

\author{
А. С. Демидов
}

\begin{abstract}
Дан обзор результатов и применяемых методов исследования, относящихся к широкому спектру задач со свободной границей для гармонических функций двух переменных. Основные результаты получены функционально-геометрическим методом. Он заключается во взаимосвязанном анализе функциональных и геометрических характеристик рассматриваемых задач и соответствующих им нелинейных задач РиманаГильберта. В обзоре приведен обширный список открытых вопросов.

Библиография: 124 названий.
\end{abstract}

Ключевые слова: свободные границы, гармонические функции.

\section{СоДЕРЖАНИЕ}

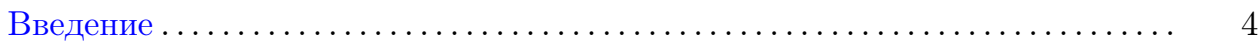

Глава I. Прямая и обратная задачи о равновесии плазмы в токамаке..... 10

$\S 1$. Теоремы о разрешимости задачи равновесия плазменного разряда при заданном топологическом типе его конфигурации ............

$\S 2$. Явная формула для гармонической функции в терминах ее данных

Коши на аналитической кривой и обратная задача о равновесии

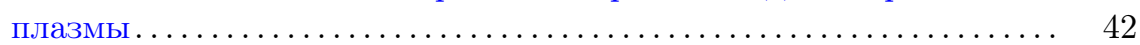

Глава II. Задача Стокса-Лейбензона (для течения Хиле-Шоу) . . . . . . . . 47

$\S 3$. Теоремы о кратких и бесконечно длительных эволюциях контура.... 47

$\S 4$. "Пальцевая" неустойчивость Кочиной-Саффмана-Тейлора ......... 53

Глава III. Задачи со свободной границей на экстремум.............. 54

$\S 5$. Плоские течения с минимальным отношением экстремальных значений давления на свободной границе..................... 54

$\S 6$. О выборе оптимальной скорости продвижения при обтекании по схеме Фёппля-Лаврентьева и об оптимизации динамики формы препят-

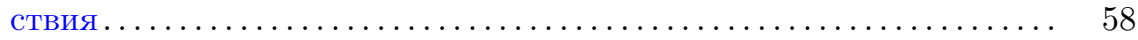

Работа выполнена при поддержке РФФИ (гранты № 05-01-22001, 07-01-00500). 
$\S 7$. Стационарное обтекание по схеме Кирхгофа криволинейного препятствия, частично абсорбирующего энергию потока, и оценка максимально возможного КПД турбины в открытом потоке..........

Глава IV. Экспоненциально точные высокочастотные асимптотики . . . . . . .

$\S 8$. Векторные поля, определяющие экспоненциально точные высокочастотные асимптотики гармонических функций............. 76

$\S 9$. Задача Олейник-Темама об усреднении ..................... 80

$\S 10$. Экспоненциально точная асимптотика гармонической функции в области с сильно гофрированной границей ............... 83

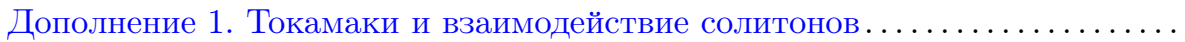

Дополнение 2. Сравнение функционально-геометрического метода и мето-

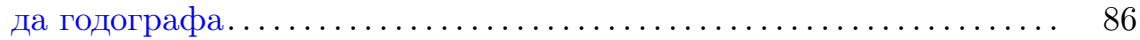

Дополнение 3. "Течение Хиле-Шоу", "Multigrid-method" и пр. .......... 88

Список литературы ........................................ 90

\section{Введение}

1. Последнее время задачам со свободными границами для гармонических функций ежегодно посвящается не менее тысячи публикаций. Интернет выдает 154 тыс. сайтов на запрос "free boundary problems for harmonic functions". Каждый год специально по этой теме проводятся несколько представительных конференций. Существенный вклад в исследования этого класса задач внесли многие замечательные ученые, среди которых Г. Гельмгольц (1821-1894), Г. Кирхгоф (1824-1887), Н. Е. Жуковский (1847-1921), М. Планк (1858-1947), С. А. Чаплыгин (1869-1942), Т. Леви-Чивита (1873-1941), Л. С. Лейбензон (1879-1951), А. Вилля (1879-1972), А. И. Некрасов (1883-1957), А. Вайнштейн (1897-1979), П. Я. Полубаринова-Кочина (1899-1999), М. А. Лаврентьев (1900-1980), Ж. Леpe (1906-1998), Л. И. Седов (1907-1999), П. П. Куфарев (1909-1968), Г. Биркгоф (1911-1996), И. И. Данилюк (1931-1988), В. И. Монахов (1932-2006), а также продолжающие ныне активно работать А. Acker, H. Berestycki, H. Brezis, L. A. Caffarelli, C. Ю. Доброхотов, В. Е. Захаров, А. Friedman, B. Gustafsson, S. D. Howison, D. Kinderlehrer, Д. В. Маклаков, М. А. Мейерманов, В. И. Налимов, L. Nirenberg, J. R. Ockendon, П. И. Плотников, G. Prokert, В. В. Пухначев, Е. В. Радкевич, S. Richardson, R. Temam, J. Serrin, G. Spruck и многие другие.

Интерес к этим задачам обусловлен их значительной ролью в изучении разнообразных важных проблем, таких как: проблемы нелинейной динамики свободной поверхности идеальной жидкости, потенциальные течения однофазных и многофазных сред, кавитационные и струйные течения, задачи фильтрации, экстремальные задачи со свободной границей для гармонических функций, . . . .

Потребность в решении такого рода задач во все более общих ситуациях необходимо влечет поиск новых подходов к их изучению. В обзоре представлены (с той или иной степенью подробности) работы многих авторов, в которых применялись разные методы исследования. Среди них метод годографа в интерпретации Кирхгофа и его последователей, методы сингулярных интегральных уравнений, а также различные вариационные методы. Но основное 
внимание в статье уделено функционально-геометрическому методу. Он заключается во взаимосвязанном анализе функциональных и геометрических характеристик рассматриваемых задач и соответствующих им нелинейных задач Римана-Гильберта. С помощью этого метода решено большинство рассмотренных в обзоре задач. Этот метод был выработан при решении $B$-задачи, или Begin-задачи, с которой началось изучение большинства представленных здесь задач. В-задача была поставлена и решена [2] в связи с вопросом Е. П. Велихова о возможности распада односвязного разряда термоядерной плазмы на отдельные компоненты связности. Математически этот вопрос можно (в простейшей ситуации) сформулировать как вопрос о существовании двусвязной области $\Omega_{1} \Subset \mathbb{R}^{2}$ и трехсвязной области $\Omega_{2} \Subset \mathbb{R}^{2}$ (см. рис. 1 ), которые обладают следующими свойствами. У них одна и та же внешняя заданная граница - кривая Г. На ней искомые в этих областях гармонические функции принимают одно и то же постоянное значение $M>0$, а интеграл вдоль $Г$ от модуля их градиента равен единице. Внутренние же, свободные границы $\gamma_{1}$ и $\gamma_{2}=\gamma_{2}^{+} \cup \gamma_{2}^{-}$ этих областей, которые охватывают искомые сечения равновесной плазмы $\omega_{1}$ и $\omega_{2}=\omega_{2}^{+} \cup \omega_{2}^{-}$(см. рис. 1$)$, должны быть нулевыми линиями уровня упомянутых гармонических функций и, сверх того, линиями уровня модулей их градиентов.

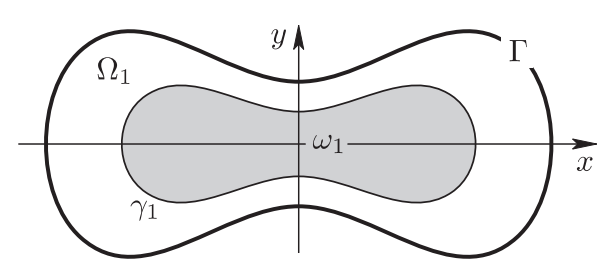

a)

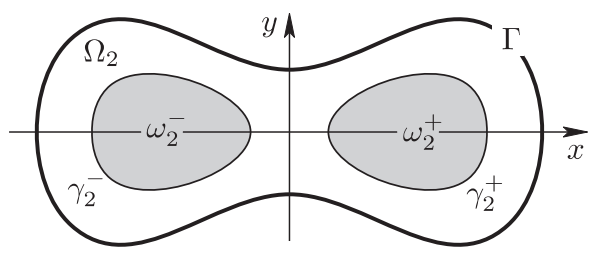

b)

Рис. 1. Два топологически различных типа а) и b) областей $\Omega_{1}$ и $\Omega_{2}$

В отношении В-задачи в статье даны необходимые, достаточные (а также некоторые необходимые и достаточные) условия на кривую Г и число $M$, при которых В-задача разрешима или неразрешима при априори заданном топологическом типе свободной границы. В случае симметрии искомых областей $\Omega_{1}$ и $\Omega_{2}$ относительно координатных осей одним из таких условий является следующее.

Условие квазивыпуклости внешней границы Г. Любая вертикаль и любая горизонталь пересекают не более чем в одной точке ту часть кривой $Г$, которая расположена в первой четверти.

Условию квазивыпуклости удовлетворяет не только любая выпуклая кривая $Г$, симметричная относительно координатных осей, но и кривые типа той, которая изображена на рис. 2.

ТЕОРема 1 [3], [4]. Пусть выполнено условие квазивыпуклости. Тогда при любом $M>0$ В-задача однозначно разрешима, если кривая $\gamma=\gamma_{1}$ гомеоморфна окружности (см. рис. 2, а), но неразрешима, если кривая $\gamma=\gamma_{2}^{+} \cup \gamma_{2}^{-}$ гомеоморфна двум внешним относителъно друг друга окружностям. 


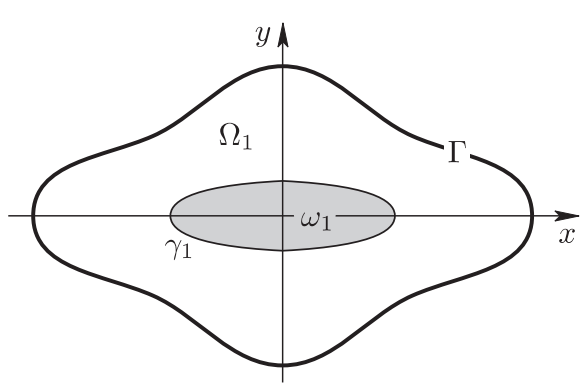

a)

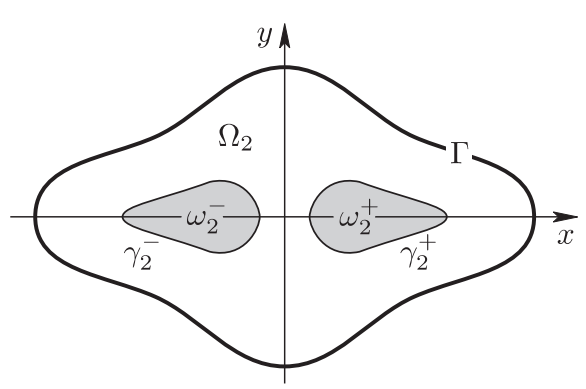

b)

Рис. 2. Когда контур Г квазивыпуклый, тогда а) В-задача однозначно разрешима для любого $M>0$, если $\left.\gamma=\gamma_{1} ; \mathrm{b}\right)$ В-задача не разрешима, если $\gamma=\gamma_{2}^{+} \cup \gamma_{2}^{-}$.

СлЕДСТВИЕ. В области $\Omega_{2}$, изображенной на рис. 2, b), В-задача неразрешима.

2. Представленные в обзоре результаты, вкратце, таковы.

В 11 изучается так называемая прямая задача о равновесии плазмы в токамаке (см. дополнение 1). Изложенная выше В-задача - ее простейший вариант. Кроме теоремы 1 , в 1 устанавливаются весьма общие условия на геометрию заданной кривой Г (симметричной относительно координатных осей), число $M>0$ и положительную функцию $q \in C[0,1]$, которые гарантируют при априори заданном топологическом типе свободной границы $\gamma$ разрешимость или неразрешимость обобщенной В-задачи, отличающейся от В-задачи лишь более общим (физически значимым) условием на свободной границе:

$$
\left.\frac{\partial u}{\partial \nu}\right|_{s \in \gamma}=\frac{q(s /|\gamma|)}{|\gamma|}, \quad \int_{0}^{1} q(t) d t=1 .
$$

Здесь $s$ - натуральный параметр на искомом контуре $\gamma,|\gamma|$ - априори не заданная длина $\gamma$, а $\partial / \partial \nu$ - производная по направлению единичной внешней нормали $\vec{\nu}$ к контуру $\gamma$.

В ряде случаев получены достаточные условия как единственности, так и неединственности искомых кривых $\gamma$ того или иного топологического типа. Эти условия выражены в терминах задаваемых параметров обобщенной В-задачи. Ими являются геометрические характеристики кривой $\Gamma$, функция $q$ и число $M$. Найдены те их значения, при которых происходит топологическая перестройка свободных границ $\gamma$. Для специального семейства внешних границ $Г$, имеющих две оси симметрии, найдены все искомые свободные границы, как симметричные, так и несимметричные относительно координатных осей. Получены достаточные условия, выраженные в терминах задаваемых параметров обобщенной В-задачи, при которых гарантированно существуют оба топологических типа свободных границ: как гомеоморфных окружности, так и гомеоморфных двум окружностям (внешним друг к другу). Эти достаточные условия дают положительный ответ на вопрос Е.П. Велихова и уточняют условия, при которых односвязный плазменный разряд, соответствующий 
кривой $\gamma_{1}$, может "развалиться" на две (быть может, более устойчивые) компоненты связности, соответствующие кривым $\gamma_{2}^{+}$и $\gamma_{2}^{-}$(как на рис. 1 ).

Сформулированная выше обобщенная В-задача относится к числу так называемых прямых задач о равновесии плазмы в токамаке. Такое название связано с тем, что задание функции $q>0$ в (1) частично определяет (см. по этому поводу, в частности, [5]-[9]) прямое, непосредственное задание правой части $F$ уравнения

$$
\frac{\partial^{2} u}{\partial x^{2}}+\frac{\partial^{2} u}{\partial y^{2}}=F(u(x, y)) \geqslant 0, \quad u \in C^{1}(\mathfrak{S}),
$$

рассматриваемого в односвязной области $\mathfrak{S}$ с заданной границей $\Gamma=\partial \mathfrak{S}$ при следующих условиях: $\left.u\right|_{\Gamma}=M>0$ и

$$
\int_{\mathfrak{S}} F(u(x, y)) d x d y=1, \quad \text { где } F(u(x, y))=0, \text { если } u(x, y)>0 .
$$

Уравнение (2), подчиненное условию (3), называется уравнением Грэда-Шафранова [10] (в так называемом цилиндрическом приближении). Уравнение (2) отражает такой важный факт магнитогидростатики: если плазменный разряд, происходящий внутри полого бесконечного цилиндра $\mathfrak{S} \times \mathbb{R}$, имеет вид соосного с ним бесконечного цилиндра $\bar{\omega} \times \mathbb{R} \subset \mathfrak{S} \times \mathbb{R}$ (с искомым сечением $\bar{\omega} \stackrel{\text { def }}{=}\{u(x, y) \leqslant 0\})$, то линии уровня функции $u: \mathfrak{S} \rightarrow \mathbb{R}$ и ее лапласиана $\Delta u: \mathfrak{S} \rightarrow \mathbb{R}$ совпадают. При этом функция $u$ есть ортогональная к $\mathfrak{S}$ компонента векторного потенциала магнитного поля.

С условием (1) связана методика изучения одной из давних проблем управляемого термоядерного синтеза. Этой проблемой является представленная в 22 обратная задача о равновесии плазмы. Она заключается в выявлении отличительных черт отображения

$$
j: \bar{\omega} \ni(x, y) \mapsto \Delta u(x, y) \stackrel{(2)}{=} F(u(x, y)),
$$

задающего распределение электрического тока $j$ в плазме. Знание этого отображения позволяет бороться с неустойчивостью плазменного разряда. Проблема, однако, в том, что функция $F$, фигурирующая в $(2)$, реально неизвестна. Впрочем, физики могут измерить $\nabla u$ на границе $\Gamma=\{u(x, y)=M\}$ области $\mathfrak{S}$. Это дает шанс изучить обратную задачу в два этапа, начав с поиска границы $\gamma$ искомой области $\bar{\omega}=\{u(x, y) \leqslant 0\}$ и вычисления $\nabla u$ на найденной свободной границе $\gamma$. Этот первый этап сводится к построению в окрестности кривой $Г$ градиента гармонической функции $u$ по ее данным Коши на любой замкнутой аналитической кривой, сколь угодно близкой к $\Gamma$. Такая явная формула для $\nabla u$ выводится в $\S 2$. Там же приведены полученные к настоящему моменту результаты, относящиеся ко второму этапу изучения обратной задачи (связанному с реконструкцией основных возможных типов функции $F$ на основе полученных в результате первого этапа данных).

В $\S 3$ и в $\S 4$ рассматривается задача Стокса-Лейбензона. Ее постановка такова. Пусть $\Omega_{0}$ - односвязная область в $\mathbb{R}^{2}$, ограниченная достаточно гладкой кривой $\Gamma_{0}$, окружающей начало координат. Закон эволюции $\Omega_{0} \rightarrow \Omega_{t}$ этой области (ее можно рассматривать как пятно жидкости) таков: в момент $t$ точка 
$\mathbf{s}(t)=(x(t), y(t))$ границы $\Gamma_{t}$ области $\Omega_{t}$ движется со скоростью $\dot{\mathbf{s}}=(\dot{x}, \dot{y})$. Эта скорость определяется кинематическим условием

$$
\dot{\mathbf{s}}=\nabla u \quad \text { на } \Gamma_{t}=\partial \Omega_{t},
$$

где $\nabla u=\left(u_{x}, u_{y}\right)$ - градиент функции $u(t ; \cdot, \cdot): \Omega_{t} \rightarrow \mathbb{R}$, удовлетворяющей условию Стокса [11]

$$
u_{x x}+u_{y y}=q \delta(x, y) \quad \text { в } \Omega_{t}
$$

и динамическому условию Лейбензона [12]

$$
u=0 \quad \text { на } \Gamma_{t} \text {. }
$$

Здесь $\delta(x, y)$ - это $\delta$-функция Дирака, сосредоточенная в начале координат, а ненулевой коэффициент $q>0$ характеризует мощность источника (при $t>0$ ) или стока (при $t<0$ ) жидкости, которой заполнена область $\Omega_{t}$. Требуется выяснить, когда эволюция $\Omega_{0} \rightarrow \Omega_{t}$ возможна при малых $t$, при больших $t$, и каковы ее характерные особенности. Задачу Стокса-Лейбензона (5)-(7) можно интерпретировать, как динамику контура $\Gamma_{t}=\partial \Omega_{t}$ нефтеносного пласта [12] или же пятна $\Omega_{t}$ зажатой между пластинами вязкой жидкости [11], [13] с нулевым поверхностным натяжением. В $§ 3$ выведено нелинейное интегро-дифференциальное уравнение, анализ которого и его матричного приближения (см. § 4) позволил выявить математический объект, свойства которого объясняют причину так называемой “пальцевой” неустойчивости Кочиной-Саффмана-Тейлора. Эта неустойчивость заключается в том, что некоторые вполне регулярные контуры $\Gamma_{0}$, охватывающие жидкость, казалось бы, ничем не отличающиеся от подавляющего большинства других, вдруг при отводе этой жидкости из скважины (расположенной в начале координат), резко меняют свою форму с образованием так называемых “языков” (в терминологии П. Я. Кочиной [14]) или "пальцев" (как стали говорить после выхода работы [15]).

В $\S 5$ рассматривается задача о поиске такой плоской криволинейной полоски $\Omega$, кромки $\gamma_{+}$и $\gamma_{-}$которой, составляющие ее границу $\gamma=\gamma_{+} \cup \gamma_{-}$, проходят через заданный дискретный набор точек и для которой функционал

$$
\Phi(\gamma)=\frac{\max _{P \in \gamma}|\nabla u(P)|}{\min _{P \in \gamma}|\nabla u(P)|}, \quad \text { где }\left.\Delta u\right|_{\Omega}=0 \text { и }\left.u\right|_{\gamma_{ \pm}}= \pm \mu,
$$

принимает минимальное значение. Здесь $\mu>0$ - заданная константа. Доказаны теоремы о разрешимости, даны конструктивные формулы для кривых $\gamma_{ \pm}$ и их асимптотических приближений при $\mu \rightarrow 0$. Эта задача связана с проблемой обледенения элементов корпуса летательных аппаратов. Речь идет о выборе такой формы полоски фольги (кромки которой, в силу конструктивных особенностей, должны проходить через заданный набор точек), чтобы минимизировать риск ее перегорания при пропускании по ней электрического тока.

В §6 рассмотрена задача об управлении скоростью движения объекта в нестационарном плоском потенциальном потоке несжимаемой жидкости в предположении о наличии в следе за движущимся объектом (искомых) точечных вихрей. Эту задачу удалось свести к стандартной задаче оптимального управления, связанной с системой обыкновенных дифференциальных уравнений. 
В $\S 7$ рассматривается задача о максимизации отбора энергии у плоского стационарного потенциального бездивергентного потока, набегающего на турбину-препятствие Г по схеме Кирхгофа (с бесконечной, заранее не заданной каверной в следе за препятствием). Это препятствие, частично поглощающее набегающий поток и, соответственно, его энергию, моделирует [16]-[18] турбину в открытом потоке реки или океанского течения. Если сопротивление, которое турбина оказывает потоку, слишком мало, то поток почти беспрепятственно просачивается. Если сопротивление слишком велико, то поток стремится обойти турбину-препятствие. В обоих этих крайних случаях поток почти не отдает энергию. Золотая середина (максимальный отбор энергии) может достигаться подходящим выбором управления, такого, например, как распределение углов

$$
\alpha: \Gamma \ni(x, y) \mapsto \alpha(x, y)
$$

входа потока в препятствие Г (технически реализуемого в виде ансамбля решеток с направляющими створками). Теоремы о разрешимости и конструктивные алгоритмы получены в $\S 7$ при весьма общем допустимом управлении (8) и достаточно произвольном виде криволинейного препятствия Г. Отметим, что в [17] (ср. также с [19]) рассматривалось препятствие в виде пластины, ортогональной симметричному потоку, а варьируемый угол $\alpha$ был постоянным на всей симметричной части этой пластины.

В §8-§10 получены явные формулы для такого аналитического отображения замкнутой аналитической кривой Г на окружность, которое удовлетворяет заданному закону продольной деформации кривой $Г$, например, ее изометрической деформации. С помощью таких явных формул строятся экспоненциально точные асимптотические приближения $u_{\varepsilon}$ для быстроосциллирующих решений $U_{\varepsilon}$ ряда краевых задач. В частности, это относится к следующей краевой задаче для уравнения Лапласа:

$$
\left.\Delta U_{\varepsilon}\right|_{\Omega}=0,\left.\quad U_{\varepsilon}\right|_{s \in \Gamma}=f\left(\frac{s}{\varepsilon}\right)
$$

в плоской области $\Omega$ с аналитической границей $\Gamma=\partial \Omega$. Здесь $s-$ натуральным параметр на $\Gamma$, а функция $f$ принадлежит соболевскому пространству $H^{\alpha-1 / 2}(\Gamma)$, где $\alpha \geqslant 0$. Доказана оценка

$$
\left\|u_{\varepsilon}-U_{\varepsilon}\right\|_{H^{\alpha}(\Omega)} \leqslant C_{\alpha} e^{-\tau / \varepsilon}, \quad \text { где } \tau>0 .
$$

Есть три отличия от традиционных построений асимптотики решения такого рода задач: 1) не исключен случай, когда граничные значения $U_{\varepsilon}$ принадлежат почти максимальному классу в шкале соболевских пространств, в которых есть единственность соответствующей гармонической функции $U_{\varepsilon} ; 2$ ) асимптотика экспоненциально близка к решению краевой задачи; 3) оценка погрешности дается в наиболее сильной норме, в которой ограничена сама функция $U_{\varepsilon}$. Дано также обобщение такого построения асимптотики для решения квазилинейного эллиптического уравнения 2-го порядка при том же краевом условии.

3. На разных этапах подготовки этого обзора существенную помощь мне оказали мои коллеги по кафедре, возглавляемой В. М. Тихомировым, и многие другие специалисты, в том числе М. С. Агранович, J. Blum, Л. Е. Захаров, 
J.-P. Lohéac, M. Moussaoui, Е. В. Панкратьев, Pierre \& Paulette Petroff, В. Д. Пустовитов, Е. В. Радкевич, M. Vogelius, В.Д. Шафранов, Э.Э. Шноль. Я им сердечно благодарен.

\section{Глава I. Прямая и обратная задачи о равновесии плазмы в токамаке}

\section{§ 1. Теоремы о разрешимости задачи равновесия плазменного разряда при заданном топологическом типе его конфигурации}

1.1. С точки зрения геометрии, камеру токамака и происходящий в ней плазменный разряд водородной плазмы (об этом см. дополнение 1) можно в первом приближении представлять в виде бесконечных цилиндров с общей образующей, которая ортогональна плоскости комплексного переменного $z=$ $x+i y \in \mathbb{C}$. Обозначим через $\mathfrak{S} \Subset \mathbb{C}$ и $\bar{\omega} \stackrel{\text { def }}{=} \omega \cup \partial \omega \Subset \mathfrak{S}$ ортогональные сечения камеры и плазменного разряда соответственно. При этом граница $\Gamma=\partial \mathfrak{S}$ односвязного сечения $\mathfrak{S}$ задана, а граница $\gamma=\partial \omega$ плазменной области $\omega$ (априори состоящей из одной или двух компонент связности) является искомой.

Как прямая, так и обратная задачи о равновесии плазмы со свободной границей $\gamma$ формулируются на базе нижеследующих соотношений (1.1)-(1.5):

$$
\begin{gathered}
\frac{\partial^{2} u}{\partial x^{2}}+\frac{\partial^{2} u}{\partial y^{2}}=F(u) \quad \text { в } \mathfrak{S}, \\
F(u(x, y))= \begin{cases}f(u(x, y)) \geqslant 0 & \text { при }(x, y) \in \bar{\omega}, \\
0 \quad \text { при }(x, y) \in \Omega^{0} \stackrel{\text { def }}{=} \mathfrak{S} \backslash \bar{\omega},\end{cases} \\
\quad \int_{\mathfrak{S}} F(u(x, y)) d x d y=I, \\
u=0 \quad \text { на свободной границе } \gamma=\partial \omega, \\
u=M \quad \text { на заданной границе } \Gamma=\partial \Omega,
\end{gathered}
$$

где (см. (1.1), (1.3), (1.5)) частное $M / I>0$ двух чисел $M>0$ и $I>0$ задано.

Уравнение (1.1) Грэда-Шафранова [10] описывает магнитную гидростатику плазменного разряда (в так называемом цилиндрическом приближении). Функция $u$ - это та компонента векторного потенциала магнитного поля, которая соосна с образующей цилиндра плазменного разряда. Уравнение (1.1) отражает то следствие магнитостатики в цилиндрической геометрии, согласно которому линии уровня функции $u: \mathfrak{S} \rightarrow \mathbb{R}$ совпадают с линиями уровня ее лапласиана. Функция $j \stackrel{(1.2)}{=} F \circ u$ задает распределение электрического тока, протекающего через сечение $\bar{\omega}$ этого разряда. Условие $f \circ u \geqslant 0$ выражает однонаправленность тока. Соотношение (1.3) задает полный ток. Его значение $I$ иногда удобно принимать за единицу измерения силы тока, а в тех случаях, когда область $\Omega^{0}=\mathfrak{S} \backslash \bar{\omega}$ симметрична относительно координатных осей $x$ и $y$, будем полагать $I=4$. Область $\Omega^{0}$ будем иногда называть вакуумной, поскольку она соответствует вакууму, окружающему плазму. Вакуум служит теплоизолятором от воздействия горячей плазмы на стенки камеры токамака. Вакуумная область $\Omega^{0}$ изолирует плазменную область $\omega$ от соприкосновения с границей $\Gamma=\partial \mathfrak{S}$. В вакууме тока нет. Поэтому $F=0$ в $\Omega^{0}$. 
Что касается давления в плазме, то его линии уровня также являются [10] линиями уровня функции $u$. При равновесии плазменного разряда давление одинаково с обеих (внешней и внутренней) сторон границы $\gamma=\partial \omega$ плазменной области $\omega$. С внешней стороны, где нет тока, оно постоянно (в силу уравнения движения). Отсюда следует, что $\gamma$ есть одна из линий уровня функции $u$. Можно считать, что $\left.u\right|_{\gamma} \stackrel{(1.4)}{=} 0$. После фиксации в (1.3) единицы измерения $I$ полного тока, число $\left.M \stackrel{(1.5)}{=} u\right|_{\Gamma}$, а потому и частное $M / I$ характеризуют отдаленность границы $\gamma$ области $\omega$ от границы $\Gamma$, представляющей сечение металлического кожуха камеры токамака. (Технически, отдаленность горячей плазмы от стенок камеры осуществляется так называемым лимитором.)

Фигурирующая в (1.1) функция $F$ определяет одну из важнейших характеристик плазменного разряда, а именно: распределение $j \stackrel{(1.2)}{=} f \circ u$ протекающего по плазме тока. Знание этой величины необходимо не только для понимания физических процессов, протекающих при разряде, но и для эффективного управления самим разрядом, для подавления неустойчивостей плазмы.

Однако эта характеристика не может быть найдена ни теоретически (в силу отсутствия соответствующей теории), ни непосредственно в эксперименте (ибо температура в плазме сравнима с температурой Солнца). Для реконструкции функции $F$ недостаточно наличия лишь системы соотношений $(1.1)-(1.5)$. Нужны дополнительные данные. Основным их источником являются данные магнитных измерений, позволяющие замерить градиент функции $u$ в ряде точек $P_{j} \in \Gamma$, где расположены датчики на кожухе камеры токамака.

Тем самым, возникает проблема поиска в искомой области $\bar{\omega}$ “существенно различных" типов априори возможных распределений

$$
j: \bar{\omega} \ni(x, y) \mapsto j(x, y) \stackrel{(1.2)}{=} f(u(x, y)),
$$

исходя из системы соотношений (1.1)-(1.5) и аппроксимативного задания нормальной производной функции $u$ в конечном числе точек $P_{j}$ кривой

$$
\Gamma=\left\{(x, y) \in \mathbb{R}^{2} \mid u(x, y)=M>0\right\} .
$$

Важность сформулированного вопроса определяется тем, что лишь одно из возможных отображений (1.6) характеризует истинное распределение тока, протекающего через сечение $\omega$ плазменного разряда. Другие же "существенно" отличные отображения никак не связаны с физикой плазмы. Если такие имеются, то их нужно отбросить, так как на их основе нельзя делать физически достоверные выводы, в том числе о таких характеристиках плазменного разряда, как ухудшение его удержания, понижение предела устойчивости, затруднение введения мощности, повышение локальных потерь на стенках камеры токамака и т. п.

Эта задача, частично поднятая в начале 70-х годов (см., например, [20]) и живо обсуждаемая физиками до сих пор (в частности, в [21]-[23]), является обратной по отношению к прямой, где ставится вопрос о поиске функции $u$, а также кривой $\gamma$, исходя из соотношений (1.1)-(1.5) при той или иной форме задания функции $f$. Прямая задача является вспомогательной при изучении основной, т. е. обратной задачи. Существенно более простая прямая задача 
позволяет ответить на вопрос о геометрии плазменной конфигурации, а также на вопрос о соответствии данных магнитной диагностики тем или иным характеристикам протекающего по плазме тока. Что касается обратной задачи, то она еще далеко не изучена. Имеющиеся к настоящему времени математические результаты по обратной задаче представлены в 2 .

1.2. Исследованию прямой задачи посвящено множество вычислительных работ физиков. Математики тоже, начиная с середины 70-х годов, внесли свой посильный вклад в изучение этой задачи со свободной границей. Здесь следует выделить два направления ${ }^{1}$.

В одном из них, представленном первоначально в работах Р. Темама и А. Берестицки \& Х. Брезиса [27]-[30], а затем и их последователей, задается функция $f$ и так или иначе анализируется вопрос существования решения $u$ задачи

$$
\frac{\partial^{2} u}{\partial x^{2}}+\frac{\partial^{2} u}{\partial y^{2}}=j(x, y) \geqslant 0 \quad \text { в } \mathfrak{S}, \quad u=M \quad \text { на } \Gamma, \quad \int_{\mathfrak{S}} j(x, y) d x d y=1,
$$

где

$$
j(x, y)= \begin{cases}f(u(x, y)) & \text { при }(x, y) \in \bar{\omega} \\ 0 & \text { при }(x, y) \in \Omega^{0}=\mathfrak{S} \backslash \bar{\omega} .\end{cases}
$$

Однако при этом возникает (отнюдь не простой) вопрос: чем является множество

$$
\mathfrak{N}=\{(x, t) \in \mathfrak{S} \mid u(x, y)=0\}
$$

нулей этого решения? По физике дела это должна быть кривая. Интерес представляет ее геометрия, ее топологический тип. Однако в работах указанного направления эти вопросы практически не изучаются. Более того, множество $\mathfrak{N}$, вообще говоря, может и не быть кривой. Это связано с тем, что в этом направлении исследований обсуждаемая постановка задачи не ориентирована на геометрию внешней (заданной) границы и особенно на геометрию и топологический тип искомого множества $\mathfrak{N}$, т. е. на то, что составляет важнейшие показатели решения исходной физической задачи. В этих условиях (не обремененных априорными требованиями к геометрии множества (1.7)) доказываются теоремы существования (какого-то) слабого решения $u$, но почти нет содержательной информации о геометрии искомой "свободной границы", точнее, о множестве $\mathfrak{N}$ нулей искомой функции. Прояснению геометрических характеристик множества $\mathfrak{N}$ в какой-то мере иногда помогают такие работы (частично инициированные задачей о равновесии плазмы), как [31]-[33] (см. также библиографию в книге [26]).

\footnotetext{
${ }^{1}$ Имеется и третье направление, представленное (прежде всего) чрезвычайно оригинальными и интересными работами Жаклин Моссино [24], [25]. Однако, вопреки интерпретации, данной ею самой, и особенно вопреки библиографическому замечанию к 4-й главе книги [26], это направление лишь номинально относится к задаче о равновесии плазмы. Дело в том, что нелинейности, которые Ж. Моссино предлагает рассматривать в правой части уравнения $\Delta u=\lambda f(u)$ для функции $u$ из соболевского пространства $H^{1}(\mathfrak{S})$ (и $\left.\lambda \in \mathbb{R}\right)$, являются композицией $g \circ \alpha$ непрерывной функции $g$ со специфическим оператором

$$
\alpha: L^{1}(\mathfrak{S}) \ni u(\cdot) \mapsto \alpha(u)(\cdot) \stackrel{\text { def }}{=} \operatorname{mes}\{y \in \mathfrak{S} \mid u(\cdot)<u(y)<0\} .
$$

Этот оператор не является ни монотонным, ни локальным, ни непрерывным, что никоим образом не оправдывает представление распределения тока $j(x, y)$ в виде $g(\alpha(u(x, y)))$.
} 
Представленное ниже другое направление исследований прямой задачи было начато в [2], [20], т. е. несколько ранее других, и оно имеет более прямое отношение к физике. Во-первых, здесь речь заранее идет о (спрямляемой) нулевой линии уровня функции $u$ заданного топологического типа, а вовсе не о каком-то множестве нулей этой функции. Во-вторых, в качестве аргумента прямой задачи здесь предъявляется вовсе не та реально неизвестная функция $f$ из $(1.2)$, вопрос о реконструкции которой является центральным в данной проблематике, а та характеристика этой функции, которая приближенно может быть найдена, а именно: $\partial u / \partial \nu$ на искомой границе $\gamma$ “вакуумной” области $\Omega^{0}$. Эти свойства в данном направлении исследований связаны с модификацией постановки прямой задачи. Акцент в ней сделан на поиск 2-связной или 3-связной области $\Omega^{0}$ при условии, что задана ее внешняя спрямляемая граница $\Gamma$, а свобода выбора ее искомой внутренней границы $\gamma$, априори спрямляемой, ограничена требованием разрешимости следующей задачи:

$$
\Delta u=0 \quad \text { в } \Omega^{0},\left.\quad u\right|_{\Gamma}=M,\left.\quad u\right|_{\gamma}=0,\left.\quad \frac{\partial u}{\partial \nu}\right|_{P_{s} \in \gamma}=\frac{q(s /|\gamma|)}{|\gamma|}, \quad \int_{0}^{1} q(t) d t=1,
$$

где $M$ - заданное положительное число, $s$ - натуральный параметр на свободной границе $\gamma$ (он отождествляется с точкой $\left.P_{s} \in \gamma\right)$, a $|\gamma|$ - априори не заданная длина $\gamma$. Как уже отмечалось, в том случае, когда область $\Omega^{0}$ априори симметрична относительно координатных осей (как на рис. 1), мы будем, удобства ради, считать, что $\int_{0}^{1} q(t) d t=I$, где $I$ равно не единице, а четырем.

Разъясним суть постановки этой задачи, ограничившись для простоты частным случаем $q \equiv 1$. (Напомним, что функция $q$, как уже отмечалась во введении, частично задает правую часть $F$ в уравнении (1.1).) Итак, пусть $q \equiv 1$. Выберем наугад искомую область $\Omega^{0}$, т. е. выберем каким-либо образом ее внутреннюю границу $\gamma$. Тогда при любой заданной константе $M>0$ найдется (единственная) функция $u$ такая, что

$$
\Delta u=0 \quad \text { в } \Omega^{0},\left.\quad u\right|_{\Gamma}=M,\left.\quad u\right|_{\gamma}=0 .
$$

Но решение $u$ этой задачи Дирихле для уравнения Лапласа в области $\Omega^{0}$, внутренняя граница $\gamma$ которой была взята наугад, практически никогда не будет удовлетворять условию

$$
\left.|\nabla u|\right|_{\gamma}=\frac{1}{|\gamma|}
$$

фигурирующему (при $q=$ const) в (1.8). В силу (1.9), $\left.u\right|_{\gamma}=0$ и $\left.\frac{\partial u}{\partial \nu}\right|_{\gamma} \geqslant 0$. Поэтому условие (1.10) означает, что

$$
\left.\frac{\partial u}{\partial \nu}\right|_{\gamma}=\text { const, } \quad \int_{\gamma} \frac{\partial u}{\partial \nu} d \gamma=1
$$

Согласно (1.11), иначе говоря, согласно (1.10), имеем:

$1)$ искомая (свободная) граница $\gamma$, будучи линией уровня решения $u$ задачи (1.9), должна быть также линией уровня модуля его градиента;

2) интеграл вдоль $\gamma$ от $|\nabla u|$ должен быть равен заданной константе. 
Важное замечание. Условие (1.10), ограничивающее свободу выбора искомой кривой $\gamma$, имеет физический смысл (о котором будет сказано несколько ниже). Если пренебречь физической аргументацией условия (1.10) и заменить его (как, например, в [34]) на более "простое"

$$
\left.\frac{\partial u}{\partial \nu}\right|_{\gamma}=C, \quad C=\text { const },
$$

которое лишь частично воспроизводит условие (1.11) (эквивалентное (1.10)), то возникнут неоправданные математические трудности, не отвечающие физическому смыслу задачи. Эти трудности возникнут даже в том случае, когда $\Gamma$ есть окружность $\left\{x^{2}+y^{2}=R^{2}\right\}$ радиуса $R=1$, а свободная граница $\gamma=\left\{x^{2}+y^{2}=\rho^{2}\right\}$ является концентричной с $\Gamma$ окружностью с искомым радиусом $\rho<1$. В этом случае оператор Лапласа принимает вид

$$
\frac{1}{r} \frac{d}{d r}\left(r \frac{d}{d r}\right), \quad \text { где } r=\sqrt{x^{2}+y^{2}} .
$$

Поэтому радиус $\rho$ искомой окружности $\gamma$ находится, как легко видеть, из уравнения $C \rho \ln (1 / \rho)=M$ с $\left.M \stackrel{(1.9)}{=} u\right|_{\Gamma}>0$. Тем самым, при $M<C / e$, где $e=2.71828 \ldots$, имеются ровно две искомые окружности $\gamma$, а при $M>C / e$ задача (1.9) со свободной границей $\gamma$, подчиненной “простому” условию (1.12), не имеет решения. К сожалению, такого рода особенности задачи (1.9) с внутренней свободной границей $\gamma$, обремененной лишь условием (1.12), иногда остаются незамеченными при проведении численных расчетов (см., например, [35]). Впрочем, таких особенностей (не адекватных физике) не возникает, если свободная граница $\gamma$ (гомеоморфная окружности) является внешней по отношению к заданной кривой Г. В этом случае задача (1.9) с условием (1.12) однозначно разрешима [36], если $C<0$. В частности, в случае концентричных окружностей $\Gamma$ и $\gamma$, соответственно заданного единичного радиуса и искомого радиуса $\rho>1$, однозначная разрешимость, т. е. однозначная разрешимость уравнения $C \rho \ln (1 / \rho)=M$, есть следствие того, что функция $\rho \mapsto \rho \ln (1 / \rho)$ строго монотонно убывает при $\rho>1$. Отметим, что эти математические результаты об однозначной разрешимости (для случая внешней свободной границы $\gamma$ ) вполне соответствуют исходной физической задаче [37], в которой свободная граница $\gamma$ является внешней относительно заданной кривой Г. Такое соответствие объясняется тем, что анализ размерностей в этой физической задаче [37] приводит к условию (1.12).

1.3. В этом пункте изложены некоторые результаты по прямой задаче. Доказательства приведены в последующих пунктах этого параграфа. Первые пять теорем относятся к таким схематично изображенным на рис. 1 (см. с. 5) и рис. 2 (см. с. 6 ) областям $\Omega^{0}=\Omega_{1}$ или $\Omega^{0}=\Omega_{2}$, которые симметричны относительно координатных осей. Эти области классифицируются по топологическому типу грании, $\gamma=\partial \omega$, охватывающих плазменные области $\omega=\omega_{k}$ с $k=1$ или с $k=2$ односвязными компонентами связности (как в [38]). Другими словами, либо $\gamma=\gamma_{1}$ гомеоморфна окружности, либо $\gamma=\gamma_{2}$ гомеоморфна объединению двух окружностей (внешних по отношению друг к другу). 
Внешняя, симметричная относительно координатных осей граница Г области $\Omega^{0}$ полностью характеризуется угловой функцией

$$
N:\left[0, \frac{|\Gamma|}{4}\right] \ni s \mapsto N(s) \in\left[N_{-}, N_{+}\right], \quad N_{-} \stackrel{\text { def }}{=} \inf N, \quad N_{+} \stackrel{\text { def }}{=} \sup N,
$$

задающей (см. рис. $6, \alpha)$ на с. 21$)$ угол $N(s)$ между осью $x$ и внешней нормалью к кривой $\Gamma$ в той точке $P_{s}$, которая расположена в первой четверти

$$
\mathbb{R}_{++}^{2} \stackrel{\text { def }}{=}\{x>0, y>0\}
$$

и которая отождествлена с соответствующим ей натуральным параметром $s$ этой кривой, отсчитываемым от точки $P_{0} \in \Gamma \cap\{x>0, y=0\}$. Отметим, что неравенство $0<N<\pi / 2$ влечет квазивыпуклость кривой $\Gamma$ (см. с. 5), а квазивыпуклость влечет неравенство $0 \leqslant N(s) \leqslant \pi / 2$ для $P_{s} \in \Gamma \cap \mathbb{R}_{++}^{2}$.

При оговоренных свойствах симметрии области $\Omega^{0}$ ее внутренняя искомая граница $\gamma$ также характеризуется своей четвертью $\gamma_{+} \stackrel{\text { def }}{=} \gamma \cap \mathbb{R}_{++}^{2}$. Поэтому задание производной $\left.\frac{\partial u}{\partial \nu}\right|_{\gamma}$, что соответствует постановке прямой задачи, достаточно осуществить лишь на $\gamma_{+}$. Это делается таким образом:

$$
\left.\frac{\partial u}{\partial \nu}\right|_{p \in \gamma_{+}}=\frac{q(r(p))}{\left|\gamma_{+}\right|}, \quad r(p)=\frac{s(p)}{\left|\gamma_{+}\right|}
$$

Здесь $q \in C^{1}[0,1]$ - заданная функция, удовлетворяющая условиям

$$
q>0, \quad \int_{0}^{1} q(r) d r=1
$$

a $s(p) \in\left[0,\left|\gamma_{+}\right|\right]$- длина дуги $\breve{p}_{0}$ контура $\gamma$, где $p_{0}$ - это точка на $\gamma$ с нулевой ординатой и максимальной абсциссой.

В нижеследующих теоремах $q^{\prime}(r) \stackrel{\text { def }}{=} \frac{d q(r)}{d r}$, а $R: v \mapsto R(v)$ - диффеоморфизм отрезка $[0,1]$, обратный к

$$
R^{-1}: r \mapsto v(r)=\int_{0}^{r} q(\xi) d \xi
$$

Теорема 1.1 (существование $\gamma_{1}$ ). Для любого числа $M>0$ существует,

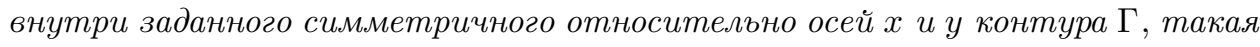
спрямляемая кривая $\gamma=\gamma_{1}$, гомеоморфная окружности и симметричная относительно осей $x$ и $y$, что в кольщеобразной области $\Omega^{0}=\Omega_{1}$ (см. рис. 1, а)), заключенной между $\gamma=\gamma_{1}$ и кривой $\Gamma$, найдется гармоническая функция $u$, удовлетворяющая условиям

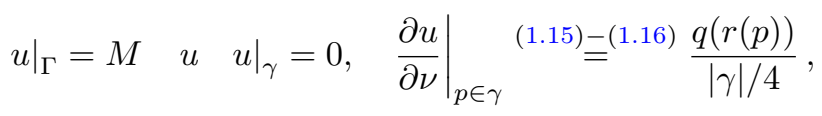

если

$$
\frac{\partial C_{+}}{\partial u}(0, v) \leqslant\left.\frac{q^{\prime}(r)}{q^{2}(r)}\right|_{r \stackrel{(1.17)}{=} R(v)} \leqslant \frac{\partial C_{-}}{\partial u}(0, v) \quad \forall v \in[0,1],
$$


где $C_{-}$и $C_{+}$- гармонические в $Q=\{0<u<M, 0<v<1\}$ функиии, подчиненные соответственно таким граничным условиям:

$$
\begin{aligned}
& C_{-}(0, v)=0, \quad C_{-}(u, 0)=0, \quad C_{-}(M, v) \stackrel{c \mathcal{M} .}{=} \stackrel{(1.13)}{=} N_{-}, \quad C_{-}(u, 1)=\frac{\pi}{2},
\end{aligned}
$$

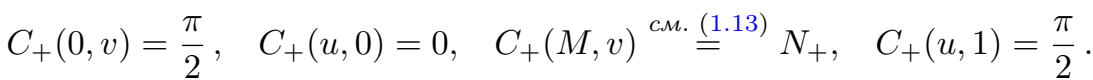

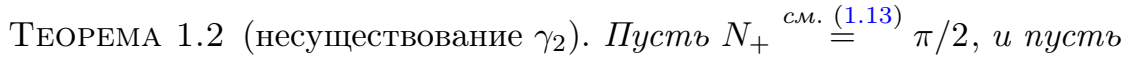

$$
0 \leqslant\left.\frac{q^{\prime}(r)}{q^{2}(r)}\right|_{r=R(v)} \leqslant \frac{\partial C_{-}}{\partial u}(0, v) \quad \forall v \in[0,1] .
$$

Тогда ни при каком $M>0$ внутри контура $\Gamma$, симметричного относительно осей $x$ и $y$, не существует спрямляемого контура $\gamma=\gamma_{2}$, симметричного относительно осей $x$ и у и гомеоморфного двум окружностям (т.е. такого, как на рис. 1, b)), для которого в области $\Omega^{0}=\Omega_{2}$, заключенной между $Г$ и $\gamma$, нашлась бы гармоническая в $\Omega^{0}$ функиия и, удовлетворяющая условиям (1.18).

Теорема 1.3 (существование $\gamma_{2}$ ). Пусть $\Gamma=\Gamma_{a}-$ такой полигональный контур длинъ $|\Gamma|=4$, для которого бункиия (1.13) принимает ровно два значения (например, как на рис. 3), а именно:

$$
N(s)= \begin{cases}N_{-} \in\left(-\frac{\pi}{2}, \frac{\pi}{2}\right), & \text { если } 0 \leqslant s<a, \\ N_{+} \in\left[\frac{\pi}{2}, \pi+N_{-}\right), & \text {если } a<s \leqslant 1=\frac{|\Gamma|}{4} .\end{cases}
$$

Пусть

$$
\frac{\partial C_{0}}{\partial u}(0, v) \leqslant\left.\frac{q^{\prime}(r)}{q^{2}(r)}\right|_{r=R(v)} \leqslant 0 \quad \forall v \in[0,1],
$$

где $C_{0}$ - гармоническая в $Q=\{0<u<M, 0<v<1\}$ функиия, подчиненная следующим граничным условиям:

$$
C_{0}(0, v)=\pi, \quad C_{0}(u, 0)=\frac{\pi}{2}, \quad C_{0}(M, v)=\max \left(N_{+}, N_{-}+\frac{\pi}{4}\right), \quad C_{0}(u, 1)=\pi .
$$

Пусть $M>0$, если $N_{-} \geqslant 0$, а если $N_{-}<0$, то пусть $M \gg 1$.

Тогда можно указать числа $\varepsilon_{*} \geqslant 0 u \varepsilon^{*} \in\left(\varepsilon_{*}, \cos N_{-}\right)$такие, что если

$$
\varepsilon_{*}<a \cos N_{-}+(1-a) \cos N_{+}<\varepsilon^{*},
$$

то найдется хотя бы один контур $\gamma=\gamma_{2}$, диффеоморфный двум окружностям, который симметричен относительно системы координат $(x, y)$ и для которого в области $\Omega^{0}=\Omega_{2}$, заключенной между $Г$ и $\gamma$, существует гармоническая в $\Omega^{0}$ функиия и, удовлетворяющая условиям (1.18).

ПРИмЕЧАНИЕ. Если $N_{+}=\pi / 2$, то $q \neq$ const ввиду неравенства (1.23). Если $q=1$ и $N_{-}>\pi / 2$, то $\varepsilon_{*}=0$. В замечании 1.5 (с. 41) даны достаточные условия существования двух различных контуров типа $\gamma_{2}$ (см. также рис. 3 и 5 ). 


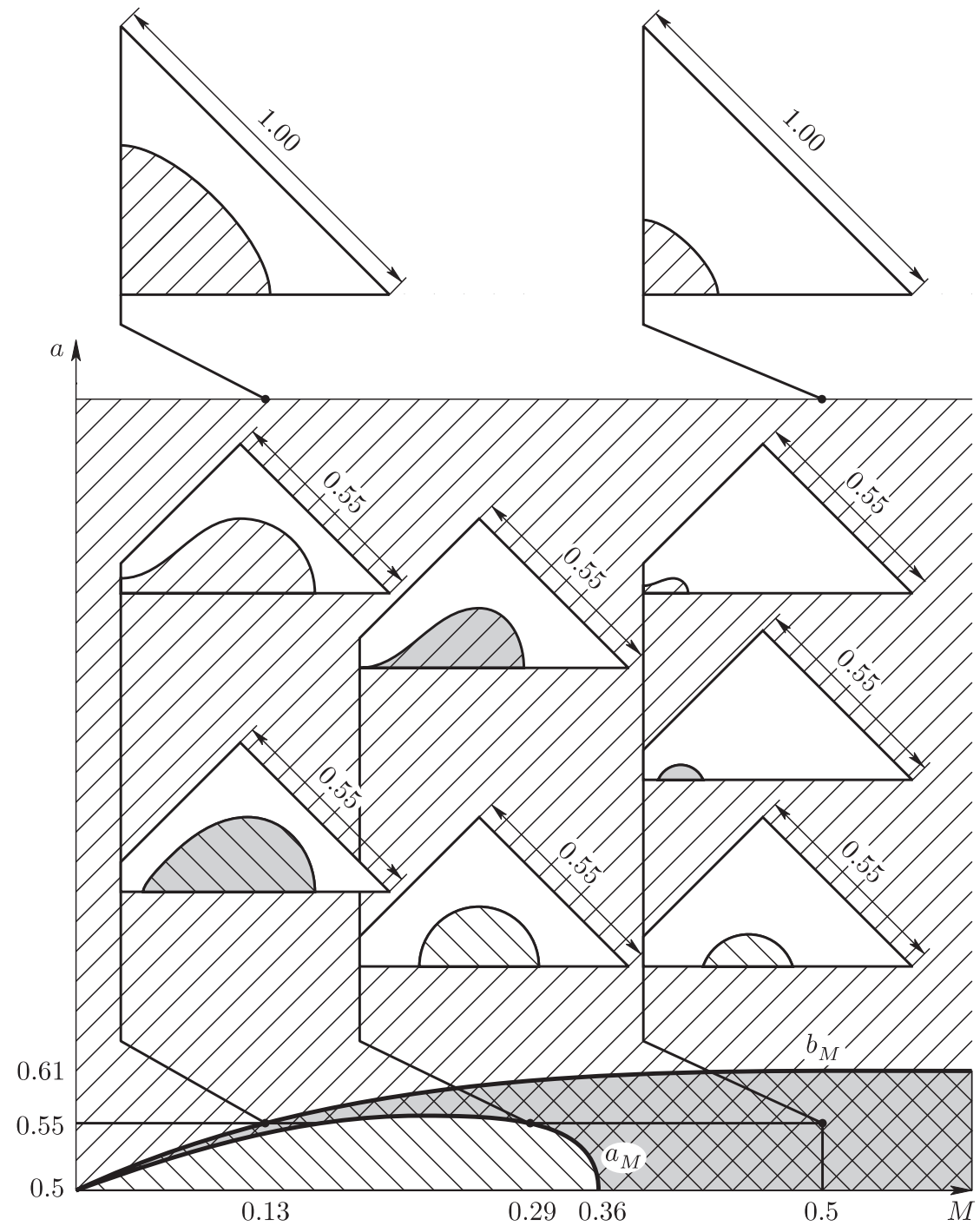

Рис. 3. Все симметричные "плазменные" конфигурации для семейства контуров $\Gamma_{a}$

Теорема 1.4 (несуществование $\gamma_{1}$ ). Пусть $\Gamma=\Gamma_{a}-$ такой же контур, как в теореме 1.3, но $N_{+} \in\left(\pi / 2, \pi+N_{-}\right)$, т.е. исключена возможность того, что $N_{+}=\pi / 2$.

Тогда для любой функиии $q \in C^{1}[0,1]$ существует $M_{0}>0$ такое, что для любого $M<M_{0}$ найдется $\varepsilon_{M}>0$ такое, что если

$$
0<a \cos N_{-}+(1-a) \cos N_{+}<\varepsilon_{M},
$$

то ни для какой кривой $\gamma=\gamma_{1}$, гомеоморфной окружности и симметричной относительно координатных осей $x$ и $y$, в области $\Omega^{0}=\Omega_{1}$, заключенной 
между Г и $\gamma$, не существует ни одной гармонической в $\Omega^{0}$ функиии, удовлетворяющей условиям (1.18).

Пересечение условий теорем 1.1 и 1.3 дает ответ на вопрос, поставленный Е. П. Велиховым. В частности, справедлива следующая теорема.

Теорема 1.5 (существование и $\gamma_{1}$, и $\gamma_{2}$ ). Пусть $q=1, a \Gamma=\Gamma_{a}$ - полигональный контур, длина $|\Gamma|$ которого равна 4 и для которого функция (1.13) принимает ровно два значения:

$$
N(s)= \begin{cases}N_{-}=\frac{\pi}{4}, & \text { если } 0 \leqslant s<a \in\left(\frac{1}{2}, 1\right], \\ N_{+}=3 \frac{\pi}{4}, & \text { если } a<s \leqslant 1=\frac{|\Gamma|}{4} .\end{cases}
$$

Пусть $b_{M}<a<a_{M}$, әде функиии $M \mapsto a_{M} u M \mapsto b_{M}$ представлены своими графиками на рис. 3. Тогда внутри Г существуют и контур $\gamma=\gamma_{1}$, и контур $\gamma=\gamma_{2}$, каждый из которых симметричен относительно системы координат $(x, y)$, причем контур $\gamma_{k}$ диффеоморфен $k$ окружностям, а в области $\Omega^{0}=\Omega_{k}$, заключенной между Г и $\gamma_{k}$, найдется гармоническая в $\Omega_{k}$ функция $u=u_{k}$, удовлетворяющая условиям (1.18).

Теорема 1.6 (существование несимметричной границы $\gamma_{1}$; [39]). Пусть $q=1, a \Gamma=\Gamma_{a}-$ контур, такой же, как в теореме 1.5. Тогда для любого $M>0$ существует $\varepsilon_{M}>0$ такое, что при $a \in\left(1 / 2,1 / 2+\varepsilon_{M}\right)$ внутри контура $\Gamma_{a}$ найдется несимметричная относительно оси $x$ аналитическая кривая $\gamma=\gamma_{1}$, диффеоморфная окружности, для которой в кольцевой области $\Omega^{0}=\Omega_{1}$, заключенной между $\Gamma_{a} u \gamma_{1}$, существует гармоническая функиия, удовлетворяющая условиям (1.18).

Численный анализ нелинейных интегральных функционалов (1.81) позволил найти все несимметричные конфигурации (см. рис. 4). Их число $k=k(a, M)$ равно 1 при $a \in\left(1 / 2, b_{M}\right)$ и 2 при $b_{M} \leqslant a<a_{M}$ (по модулю отражений относительно оси $y$ ). Бифуркационные связи симметричных и несимметричных конфигураций $\gamma$ в случае $q=1$ и семейства контуров $\Gamma_{a}$, описанных в теоремах 1.5 и 1.6, представлены на рис. 5.

Без каких-либо предположений о симметрии контура Г справедлива следующая теорема.

ТеОрема 1.7 (существование $\gamma_{1}$, когда Г лишь выпукла). Если $Г$ - выпуклая кривая, то для любого $M>0$ внутри $\Gamma$ существует кривая $\gamma=\gamma_{1}$, гомеоморфная окружности, для которой в кольцевой области $\Omega^{0}$, заключенной между Г и, , существует гармоническая функция такая, что ${ }^{2}$

$$
\left.u\right|_{\Gamma}=M,\left.\quad u\right|_{\gamma}=\left.0 \quad u \quad \frac{\partial u}{\partial \nu}\right|_{\gamma}=\frac{1}{|\gamma|} .
$$

При этом кривая $\gamma$ необходимо выпукла и аналитична.

\footnotetext{
${ }^{2}$ В кандидатской диссертации В. В. Петровой (см. также [40]) теорема 1.7 обобщена на случай, когда условие $\left.\frac{\partial u}{\partial \nu}\right|_{\gamma}=\frac{1}{|\gamma|}$ заменено на более общее, типа (1.15), с некоторыми ограничениями на функцию $q$, обеспечивающими выпуклость искомой кривой $\gamma$.
} 

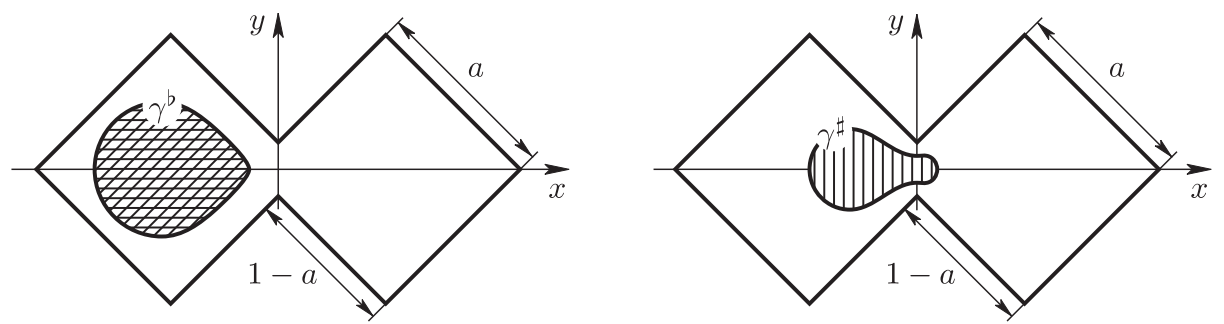

Рис. 4. Несимметричные конфигурации $\gamma^{b}$ и $\gamma^{\#}$ (при $a=0.54$ и $M=0.13$ )
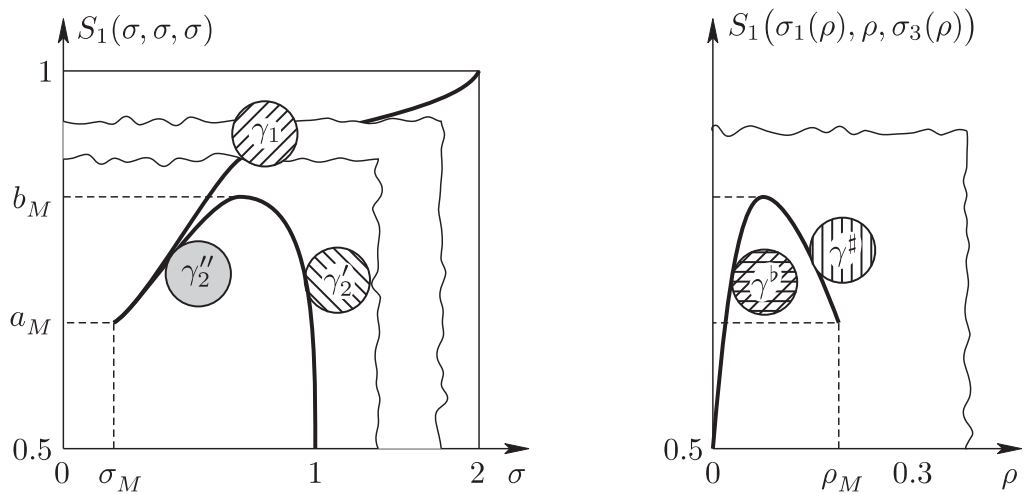

Рис. 5. Типичные графики функций $\sigma \mapsto S_{1}(\sigma, \sigma, \sigma)$ и $\rho \mapsto S_{1}\left(\sigma_{1}(\rho)\right.$, $\left.\rho, \sigma_{3}(\rho)\right)$, определенных в (1.81). Корни уравнения $a=S_{1}(\sigma, \sigma, \sigma)$ определяют симметричные "плазменные" конфигурации $\gamma_{1}, \gamma_{2}^{\prime}, \gamma_{2}^{\prime \prime}$, а корни уравнения $a=S_{1}\left(\sigma_{1}(\rho), \rho, \sigma_{3}(\rho)\right)$ определяют несимметричные конфигурации $\gamma^{b}$ и $\gamma^{\#}$.

1.4. Общая схема доказательств основных теорем, представленных в обзоре, в том числе теорем 1.1-1.7, включает в себя три этапа. На первом этапе каждой рассматриваемой задаче ставится в соответствие эквивалентная ей задача, которую можно отнести к классу $\Gamma K$-задач, определяемому чуть ниже. Он назван здесь так в честь Гельмгольца, предложившего [1] их вниманию исследователей ${ }^{3}$, и Кирхгофа [43], который выдвинул идею, способствовавшую их активному изучению (см. дополнение 2). На втором этапе возникшей ГК-задаче сопо-

\footnotetext{
${ }^{3}$ При этом Гельмгольц подчеркивал [1], что он дает лишь “набросок математической обработки" задач нового типа. Сам Гельмгольц не имел времени для разработки предложенной им темы. Его ум работал во многих направлениях: физиологическая оптика и акустика, электродинамика и термодинамика, закон сохранения энергии и принцип наименьшего действия, теория вихрей и т. д. Значимость его работ в этих направлениях была столь велика, что постоянно цитируемая здесь работа [1] представлялась на их фоне как бы незначительной заметкой (биографы упоминают о ней вскользь, работа [1] не вошла в собрание [41] его сочинений). Примечательно одно обстоятельство, которое в какой-то мере явилось причиной необычайной широты научных исследований Гельмгольца. О нем Гельмгольц поведал в застольной речи на праздновании своего 70-летия (перевод по книге [42; с. 188]): "Наступало время перехода в университет. В ту пору физика еще не считалась в числе хлебных занятий. Мои родители были вынуждены жить крайне бережливо. Отец объявил, что может помочь мне в изучении физики не иначе как под условием, что я возьму и медицину в придачу. Я был ничуть не против того, чтобы изучать живую природу, и согласился без затруднений”.
} 
ставляется некоторая (нелинейная) задача Римана-Гильберта. Это осуществляется с помощью двух базовых идей Гельмгольца и Кирхгофа. Они сформулированы несколько ниже и объединены здесь под названием “ $Г K$-метод", что означает "метод Гельмгольца-Кирхгофа". Заключительный, третий этап доказательств связан с исследованием этой задачи Римана-Гильберта функционально-геометрическим методом. Вкратце о нем сказано в конце этого пункта.

В ГK-задачах искомъми являются область $\Omega \subset \mathbb{R}^{2}$ и однолистное отображение

$$
w: \Omega \ni z=x+i y \mapsto w(z)=u(x, y)+i v(x, y) \in Q
$$

на некоторую (как правило) априори известную односвязную область $Q$. Отображение (1.26) подчинено в каждой конкретной задаче тем или иным дополнительным условиям.

Отметим, что обычно (например, как в В-задаче из введения) область $\Omega$, о которой идет речь в ГК-задаче, явно не фигурирует в качестве объекта исходной задачи. Эту область $\Omega$ нужно предварительно еще выявить.

Как уже было сказано, подход к исследованию ГК-задач, названный в обзоре ГК-методом, базируется на двух идеях, выдвинутых Гельмгольцем и Кирхгофом.

Первая идея, непосредственно связанная с самим понятием ГК-задачи, принадлежит Гельмгольцу [1]. Он выразил ее (с точностью до обозначений) следующим образом: “Величины $u$ и $v$ рассматривают обычно как функции переменных $x$ и $y$. Но можно, наоборот, $z=x+i y \in \Omega$ выражать как функцию от $w=u+i v \in Q$ и разыскивать решение в такой форме".

Суть этой идеи Гельмгольца состоит в сведении ГК-задачи к задаче в фuксированной области $Q=w(\Omega)$. Рецепт, позволяющий ее изучить, дает

Вторая идея. Едва уловимая в формулах, полученных Гельмгольцем [1], эта идея отчетливо сформулирована Кирхгофом [43]. По существу, она состоит в предложении изучать в фиксированной области $Q$ функцию

$$
A+i B: Q \ni w=u+i v \mapsto A(u, v)+i B(u, v) \stackrel{\text { def }}{=} \ln \frac{d z}{d w} \in \mathbb{C}, \quad z=x+i y .
$$

Роль функции $A+i B$, названной в обзоре $\Gamma$ К-функцией (или функцией Гельмгольца-Кирхгофа), определена двумя обстоятельствами:

1) согласно формуле Ньютона-Лейбница, искомая область дается формулой

$$
\Omega=\left\{z(w)=z_{0}+\int_{w_{0}}^{w} e^{A(\xi, \eta)+i B(\xi, \eta)} d \zeta, \zeta=\xi+\eta, w \in Q, z_{0}=z\left(w_{0}\right)\right\}
$$

2) известный геометрический смысл вещественной и мнимой частей функции $A+i B=\ln (d z / d w)$ позволяет выписать необходимые условия разрешимости исходной задачи в терминах (вообще говоря, нелинейной) задачи Римана-Гильберта в фиксированной области $Q$ относительно функции $A+i B$.

$\mathrm{K}$ примеру, как вскоре мы увидим, необходимым условием разрешимости В-задачи является существование в изображенном на рис. $6, \beta$ ) прямоугольнике

$$
Q=\{w=u+i v \in \mathbb{C} \mid 0<u<M, 0<v<1\}
$$




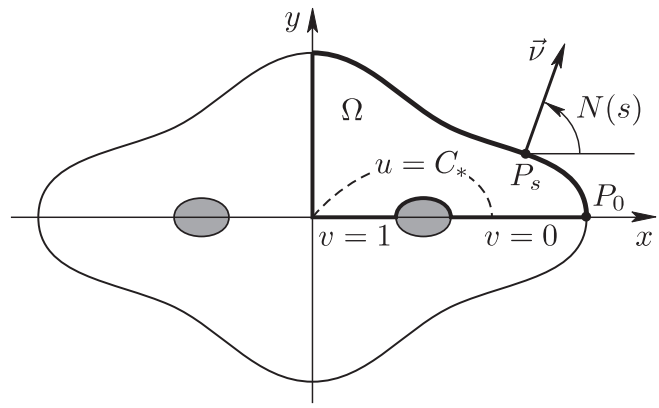

$\alpha)$

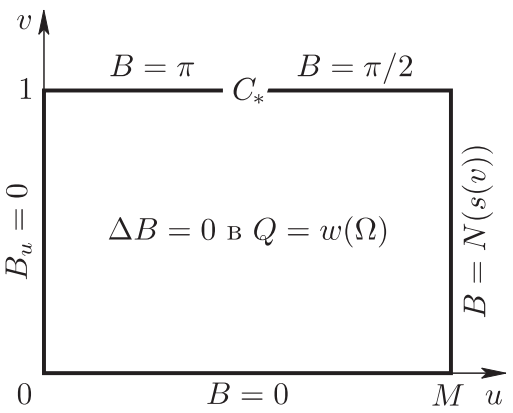

$\beta)$

Рис. 6. $\alpha$ ) Гипотетически возможная область типа $\Omega_{2}$, внешняя граница которой в первой четверти подчинена геометрическому условию: $0 \leqslant N(s) \leqslant \pi / 2$. $\beta$ ) Соответствующая В-задаче нелинейная задача Римана-Гильберта в прямоугольнике $Q=w(\Omega)$, где $\Omega=\Omega_{k} \cap \mathbb{R}_{++}^{2}$, а $w=u+i v: \Omega \rightarrow Q-$ аналитическая функция.

такой аналитической функции $A+i B: Q \rightarrow \mathbb{C}$, которая ограничена в замыкании области $Q$ и подчинена следующим нелинейным граничным условиям:

$$
B(u, 0)=0, \quad B(M, v)=\varphi(v), \quad B(u, 1)=\psi_{*}(u), \quad B_{u}(0, v)=0 .
$$

Здесь $\varphi(v)=N(s(v)), s(v)=\int_{0}^{v} e^{A(M, \eta)} d \eta, s(1)=|\Gamma| / 4, \mathrm{a}$

$$
\psi_{*}(u)=\frac{\pi}{2} \quad \text { при } C_{*}<u<M, \quad \psi_{*}(u)=\pi \quad \text { при } 0<u<C_{*},
$$

где число $C_{*}$ (заключенное между нулем и $M$ ) характеризуется тем, что для случая $k=1$ (см. рис. 1, а))

$$
C_{*}=0 \quad \text { и } \quad J(v)>0 \quad \text { при } 0<v \leqslant 1,
$$

а в случае $k=2$ (см. рис. $1, \mathrm{~b}))$ выполнено нелинейное ограничение

$$
J(v) \stackrel{\text { def }}{=} \int_{0}^{v} \cos B(0, \eta) d \eta \begin{cases}=0 & \text { при } v=1, \\ >0 & \text { при } v \in(0,1) .\end{cases}
$$

Невыполнение необходимых условий разрешимости изучаемой задачи влечет ее неразрешимость. Так, например, В-задача неразрешима в случае кривой $\gamma=\gamma_{2}$, поскольку нарушено [2], [44] необходимое условие $J(1) \stackrel{(1.32)}{=} 0$ разрешимости, если $0 \leqslant N(s) \leqslant \pi / 2$. Доказательство приведено в п. 1.8.

Функционально-геометрический метод есть совокупность взаимосвязанных функциональных и геометрических построений, которые позволяют:

1) сформулировать в полной мере (нелинейную и/или с нелинейными ограничениями) задачу Римана-Гильберта, соответствующую ГК-задаче, и

2) проанализировать эту задачу Римана-Гильберта, доказав теоремы о ее разрешимости (или неразрешимости), установив в итоге для исходной задачи 
со свободной границей результаты, касающиеся разрешимости, а в ряде случаев изучив и вопросы единственности, бифуркации ее решения, а также выявив некоторые качественные его характеристики.

Конечно, упомянутая совокупность функциональных и геометрических построений может иметь свои особенности при доказательстве конкретных теорем. В отношении теорем 1.1-1.7 эти особенности представлены в последующих пунктах этого параграфа. Одна из таких особенностей заключается в том, что существенно используется априорная информация о геометрии кривых $Г$ и $\gamma$.

1.5. Все доказательства начинаются с проверки того, что каждая изучаемая в этом параграфе задача сводится к ГК-задаче.

Рассмотрим сначала случай, относящийся к теореме 1.7 , т. е. к случаю, когда априори предполагается только выпуклость кривой $\Gamma$, а искомая кривая $\gamma$ (лежащая внутри Г) гомеоморфна окружности. Возьмем произвольную точку $P_{0} \in \Gamma$ (см. рис. 7, a)). От нее отложим в положительном направлении нату-

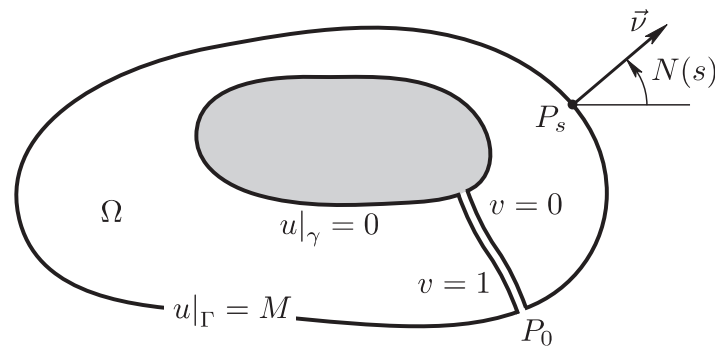

a)

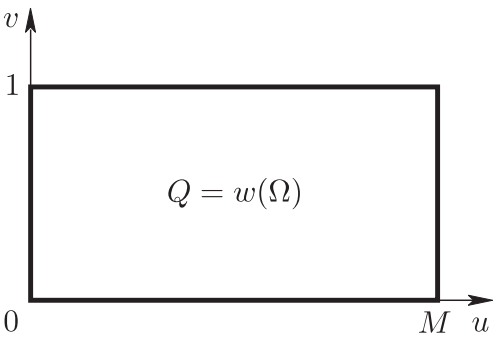

b)

Рис. 7. а) Область $\Omega=\Omega(\gamma) ; N(s)$ - угол между осью $x$ и нормалью $\vec{\nu}$ к $\Gamma$ в точке $P_{s} \in \Gamma$. b) Образ $Q=w(\Omega)$ области $\Omega$ при однолистном отображении $w=u+i v$.

ральный параметр $s$, т. е. длину дуги $P_{0} P_{s}$ кривой $\Gamma$. Обозначим через $N(s)$ угол между осью $x$ и нормалью $\vec{\nu}$ к $\Gamma$ в точке $P_{s} \in \Gamma$. Кривая Г полностью определяется угловой функиией $N:[0,|\Gamma|) \ni s \mapsto N(s)$. Предположим, что рассматриваемая задача имеет решение. Проведем через точку $P_{0}$ ту кривую $l$, которой касается $\nabla u$. Кривая $l$ пересечет свободную границу $\gamma$. Действительно [45], при конформном отображении области $\Omega^{0}$ на кольцо линии уровня функции $u$ перейдут в концентрические окружности, а образ кривой $l$ будет им ортогонален. Вдоль кривой $l$ сделаем разрез и в образовавшейся односвязной области $\Omega=\Omega^{0} \backslash l$ введем функцию $v$, гармонически-сопряженную с $u$. Функция $v$ определена с точностью до константы, а на каждом берегу разреза вдоль кривой $l$ она постоянна (в силу условий Коши-Римана). Можно считать, что $v \geqslant 0$ и что на одном из берегов разреза она равна нулю. Выбрав тот (определенный почти всюду) единичный касательный вектор $\tau$ к (спрямляемой) кривой $\gamma$, при котором пара $(\tau, \nu)$ положительно ориентирована, и проинтегрировав производную $\partial v / \partial \tau$ вдоль $\gamma$ (от точки пересечения кривых $l$ и $\gamma$, в которой $v=0$ ), получим значение функции $v$ на другом берегу разреза. Оно, в силу условия (1.25), равно $I=1$ (так как $\partial v / \partial \tau=\partial u / \partial \nu)$. Положим (см. рис. $7, \mathrm{~b}))$

$$
Q=\{w=u+i v \in \mathbb{C} \mid 0<u<M, 0<v<1\} .
$$


Заметим, что функция

$$
w: \Omega \ni z=x+i y \mapsto w(z)=u(x, y)+i v(x, y) \in Q
$$

однолистно отображает искомую область $\Omega$ со свободной границей $\gamma$ на односвязную фиксированную область $Q$. Однолистность следует из принципа соответствия границ [46]. Согласно этой теореме, достаточно проверить, что аналитическое отображение непрерывно вплоть до границы области и гомеоморфно с сохранением ориентации отображает границу. Последнее сразу следует из конформной эквивалентности области $\Omega^{0}$ кольцу [45], теоремы о соответствии границ [46] и того, что максимум функции $u$ достигается лишь на $Г$.

Итак, мы проверили, что рассматриваемая в теореме 1.7 задача сводится к некоторой ГК-задаче. Еще проще такого рода факт устанавливается в том случае, когда условие выпуклости области $\Omega^{0}=\Omega_{k}$ заменяется условием ее симметрии относительно координатных осей. В этом случае можно взять $\Omega \stackrel{(1.14)}{=} \Omega^{0} \cap \mathbb{R}_{++}^{2}$. При этом область $Q=w(\Omega)$ есть прямоугольник (1.33).

1.6. Ниже нам понадобятся следующие две леммы.

Лемма 1.1. Пусть $\left\{f_{n}\right\}_{n \in \mathbb{N}}-$ последовательность функиий, ограниченных в $L^{p}(\Omega)$, где $p \in(1, \infty)$, а $\Omega \subset \mathbb{R}^{k}$ - множество конечной мерь. Пусть $\left\{f_{n}\right\}$ сходится по мере $\kappa f \in L^{1}(\Omega)$. Тогда

$$
\int_{\Omega} f_{n}(x) d x \rightarrow \int_{\Omega} f(x) d x \quad \text { nрu } n \rightarrow \infty .
$$

ДокАЗАТЕЛЬство. Функции $f_{n}$ имеют равностепенно абсолютно непрерывные интегралы, так как

$$
\left|\int_{\sigma} f_{n}(x) d x\right| \leqslant C(\operatorname{mes}(\sigma))^{1 / q}, \quad \text { где } \frac{1}{q}=1-\frac{1}{p},
$$

a

$$
C^{p}=\sup _{n} \int_{\Omega}\left|f_{n}(x)\right|^{p} d x<\infty .
$$

Поэтому лемма следует из известной теоремы Витали [47].

Рассмотрим теперь в прямоугольнике (1.33) смешанную краевую задачу

$$
\Delta \beta=0 \quad \text { в } Q,\left.\quad \frac{\partial \beta}{\partial u}\right|_{\partial_{I}}=\psi,\left.\quad \beta\right|_{\partial_{J}}=\varphi
$$

для уравнения Лапласа, где

$$
\partial_{I}=\left\{(u, v) \in \mathbb{R}^{2} \mid u=0,0<v<1\right\}, \quad \partial_{J}=\partial Q \backslash \overline{\partial_{I}} .
$$

Лемма 1.2 (см. [48]). Пустъ $\psi \in C[0,1]$, а функиия $\varphi \in C\left(\partial_{J}\right)$ имеет почти всюду производную $\varphi^{\prime} \in L^{\infty}\left(\partial_{J}\right)$. Тогда задача (1.34) имеет единственное ограниченное решение $\beta \in C(Q)$ и $\partial \beta / \partial u \in C\left(\bar{Q} \backslash \overline{\partial_{J}}\right)$. Кроме того, существует такая постоянная $C$, что

$$
\left|\int_{u_{1}}^{u_{2}} \frac{\partial \beta}{\partial v}(\xi, v) d \xi\right| \leqslant C \quad \forall u_{1}, u_{2} \in[0,1], \quad v \in(0,1) .
$$

Далее, если $\psi \in C^{k-1, \lambda}[0,1]$, где $k \geqslant 1, a \lambda \in(0,1)$, mо $\beta \in C^{k-1, \lambda}\left(\bar{Q} \backslash \overline{\partial_{J}}\right)$. 
ЗАмечАНИЕ 1.1. Пусть в задаче $(1.34) \psi=0, \varphi(\cdot, 0)=N_{0}, \varphi(\cdot, 0)=$ $N_{m+2}$, a

$$
\varphi(1, \cdot)=\sum_{j=1}^{m+1} N_{j} 1_{\left(\sigma_{j-1}, \sigma_{j}\right)(\cdot)},
$$

где $N_{j}$ - некоторые постоянные, $1_{(a, b)}$ - характеристическая функция интервала $(a, b)$, а $\sigma=\left(\sigma_{1}, \ldots, \sigma_{m}\right)$ - такой мультипараметр, что

$$
0=\sigma_{0}<\sigma_{1}<\cdots<\sigma_{m}<\sigma_{m+1}=1 .
$$

Тогда, как нетрудно установить [48], справедлива следующая формула:

$$
\begin{aligned}
\exp \{ & \left.\int_{0}^{u} \frac{\partial \beta}{\partial v}(\xi, v) d \xi\right\} \\
& =\prod_{j=0}^{m+1}\left(\frac{\left[y^{2}(u, v)+\left(x(u, v)+a_{j}\right)^{2}\right]\left[y^{2}(0, v)+\left(x(0, v)-a_{j}\right)^{2}\right]}{\left[y^{2}(u, v)+\left(x(u, v)-a_{j}\right)^{2}\right]\left[y^{2}(0, v)+\left(x(0, v)+a_{j}\right)^{2}\right]}\right)^{\delta N_{j} / 2},
\end{aligned}
$$

где $z: w \mapsto z(w)=x(u, v)+i y(u, v)$ - конформное отображение прямоугольника $Q$ на полуплоскость $\mathbb{R}_{+}^{2}, a_{j}=x\left(M, \sigma_{j}\right)$, а $\delta N_{j}=\left(\delta N_{j+1}-\delta N_{j}\right) / \pi$. Обозначая через $E(u, v)$ правую часть формулы $(1.38)$, замечаем, что если $\delta N_{j}<1$, то $E(u, \cdot) \in L^{1}(0,1)$ для всех $u \in(0, M)$ и $E(\cdot, v) \in L^{1}(0, M)$ для всех $v \in(0,1)$.

1.7. Выведем необходимые условия разрешимости сформулированных выше задач о существовании свободных границ $\gamma_{1}$ и $\gamma_{2}$ областей $\Omega_{1}$ и $\Omega_{2}$ (упомянутых во введении), которые имеют две оси симметрии, а именно координатные оси $x$ и $y$. Обозначим через $v$ такую определенную в $\Omega_{k} \cap \mathbb{R}_{++}^{2}$ гармонически-сопряженную к $u$ функцию, которая равна нулю при $y=0$. Положим $w(z)=u(x, y)+i v(x, y), z=x+i y$. Как уже было отмечено, аналитическая функция $w$ биективно отображает $\Omega_{k} \cap \mathbb{R}_{++}^{2}$ на прямоугольник (1.33). В $Q$ введем аналитическую функцию

$$
A+i B: Q \ni w \mapsto A(u, v)+i B(u, v)=\ln \frac{d z}{d w} .
$$

ПреДЛОЖЕНИЕ 1.1. Функиия В является решением следующей задачи:

$$
\left.\Delta B\right|_{Q}=0,\left.\quad B\right|_{v=0}=0,\left.\quad B\right|_{u=M}=\varphi,\left.\quad B\right|_{v=1}=\chi,\left.\quad \frac{\partial B}{\partial u}\right|_{u=0}=\psi .
$$

Здесь (см. рис. 6)

1) $\varphi(v)=N(s(v)), s(v)=\int_{0}^{v} e^{A(M, \eta)} d \eta, s(1)=|\Gamma| / 4$;

2) $\chi=\pi / 2$, если $\gamma=\gamma_{1}$, а если $\gamma=\gamma_{2}$, то $\chi(u)=\pi$ при $0<u<C_{*}$ и $\chi(u)=\pi / 2$ при $C_{*}<u<M$, где $C_{*}=u(0,0)$ (cм. рис. 6, $\beta$ ) на с. 21);

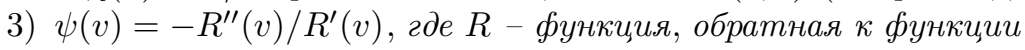

$$
R^{-1}:[0,1] \ni r \stackrel{c \mathcal{M} .}{\mapsto(1.17)} v(r)=\int_{0}^{r} q(\xi) d \xi
$$

(u, тем самым, определенная на $[0,1])$. 
Кроме того,

$$
J(v) \stackrel{\text { def }}{=} \int_{0}^{v} R^{\prime}(\eta) \cos B(0, \eta) d \eta>0 \quad \forall v \in(0,1] \quad \text { в случае } \gamma=\gamma_{1}
$$

$u$

$$
J(v)>0 \quad \forall v \in(0,1), \quad J(1)=0 \quad \text { в случае } \gamma=\gamma_{2} .
$$

ДокАзАтЕльство. Не вполне очевидны лишь формулы для функции $\chi$ и для интеграла $J$. Для вывода этих формул заметим, что функция $R$ является решением задачи

$$
\frac{d R}{d v}=\frac{1}{q(r)}, \quad R(0)=0, \quad \text { где } v(r)=\int_{0}^{r} q(\xi) d \xi .
$$

Поэтому для любого $v \in(0,1)$ имеем:

$$
\lim _{u \rightarrow+0} A(u, v)=-\lim _{u \rightarrow+0} \ln \left|\frac{d w}{d z}\right|_{z=z(u+i v)} \mid=\ln \left(|\gamma| R^{\prime}(v)\right) .
$$

Так как $q \in C^{1}[0,1]$, то $\frac{\partial A}{\partial v} \in C\left(\bar{Q} \backslash \partial_{J}\right)$, где $\partial_{J}$ определено в (1.35). Отсюда получаем

$$
\lim _{u \rightarrow+0} \frac{\partial B(u, v)}{\partial u}=-\lim _{u \rightarrow+0} \frac{\partial A(u, v)}{\partial v}=-\frac{R^{\prime \prime}(v)}{R^{\prime}(v)}, \quad 0<v<1 .
$$

Неравенства (1.40) и (1.41) вытекают из следующих формул:

$$
\begin{aligned}
\operatorname{Im}(z(i v)-z(0)) & =\operatorname{Im} \int_{0}^{i v} \frac{d z}{d w} d w=\operatorname{Im} \int_{0}^{v} i e^{A(0, \eta)+i B(0, \eta)} d \eta \\
& =\frac{e^{A(0,0)}}{R^{\prime}(0)} \int_{0}^{v} R^{\prime}(\eta) \cos B(0, \eta) d \eta=\frac{e^{A(0,0)}}{R^{\prime}(0)} J(v) .
\end{aligned}
$$

Предложение 1.1 доказано.

Ниже мы будем применять очевидное тождество

$$
-\frac{R^{\prime \prime}(v)}{R^{\prime}}(v)=\frac{q^{\prime}(r)}{q^{2}(r)}, \quad \text { где } v=\int_{0}^{r} q(\xi) d \xi, \text { а } q^{\prime}(r)=\frac{d q(r)}{d r},
$$

и следующую простую лемму.

ЛЕмма 1.3. Если функиия $\beta \in C\left(\bar{Q} \backslash \bar{\partial}_{J}\right)$ является решением задачи (1.34), где $\varphi=0, a \psi \geqslant 0$, mo $\beta \leqslant 0$.

1.8. Теперь нетрудно провести докАЗАТЕльСтво ТЕОРЕмы 1.2 об условиях, при которых невозможен распад плазмы на две компоненты связности.

Пусть $\psi=-R^{\prime \prime} / R^{\prime}$, а функция $B$ - решение задачи (1.39). Рассмотрим гармонические в $Q$ функции $B_{-}, B_{+}$и $D_{+}$, удовлетворяющие следующим граничным условиям:

$$
\begin{aligned}
& \left.B_{-}\right|_{v=0}=0,\left.\quad B_{-}\right|_{u=M} \stackrel{(1.13)}{=} N_{-},\left.\quad B_{-}\right|_{v=1}=\frac{\pi}{2},\left.\quad \frac{\partial B_{-}}{\partial u}\right|_{u=0}=\psi, \\
& \left.B_{+}\right|_{v=0}=0,\left.\quad B_{+}\right|_{u=M} \stackrel{(1.13)}{=} N_{+},\left.\quad B_{+}\right|_{v=1}=\frac{\pi}{2},\left.\quad \frac{\partial B_{+}}{\partial u}\right|_{u=0}=\psi, \\
& \left.D_{+}\right|_{v=0}=0,\left.\quad D_{+}\right|_{u=M}=\frac{\pi}{2},\left.\quad D_{+}\right|_{v=1}=\pi,\left.\quad \frac{\partial D_{+}}{\partial u}\right|_{u=0}=0 .
\end{aligned}
$$


В силу сделанных предположений и леммы 1.3, имеем

$$
0 \stackrel{(1.20)}{=} C_{-}(0, v) \leqslant B_{-}(0, v)<B(0, v)<B_{+}(0, v) \leqslant D_{+}(0, v) \leqslant \pi
$$

для любого $v \in(0,1)$. Отсюда

$$
\int_{0}^{1} R^{\prime}(v) \cos B(0, v) d v>\int_{0}^{1} R^{\prime}(v) \cos D_{+}(0, v) d v .
$$

Учитывая очевидную симметрию функции $D_{+}(0, \cdot)$ и невозрастание $R^{\prime}$, имеем

$$
\begin{aligned}
\int_{0}^{1 / 2} R^{\prime}(v) \cos D_{+}(0, v) d v & >\int_{0}^{1 / 2} R^{\prime}\left(\frac{1}{2}\right) \cos D_{+}(0, v) d v \\
\int_{1 / 2}^{1} R^{\prime}(v) \cos D_{+}(0, v) d v & >\int_{1 / 2}^{1} R^{\prime}\left(\frac{1}{2}\right) \cos D_{+}(0, v) d v .
\end{aligned}
$$

В итоге получаем

$$
\int_{0}^{1} R^{\prime}(v) \cos B(0, v) d v>R^{\prime}\left(\frac{1}{2}\right) \int_{0}^{1} \cos D_{+}(0, v) d v=0,
$$

т. е. необходимое условие (1.41) нарушено. Теорема 1.2 доказана.

ЗАмечАниЕ 1.2. Условие (1.22) непротиворечиво, если $\partial C_{-} / \partial u \geqslant 0$. Последнее верно при любом $M$, если $N_{-} \geqslant 0$, а при $M \gg 1$ и в случае $N_{-}<0$.

ЗАмечАние 1.3 . Если $N_{-} \geqslant 0$, то $\frac{\partial C_{-}}{\partial u}(0, \cdot) \geqslant \rho$, где $\rho=\frac{\partial \alpha}{\partial u}(0, v)$, а $\alpha-$ решение задачи

$$
\left.\Delta \alpha\right|_{Q}=0,\left.\quad \alpha\right|_{v=0}=0,\left.\quad \alpha\right|_{u=M}=0,\left.\quad \alpha\right|_{v=1}=\frac{\pi}{2},\left.\quad \alpha\right|_{u=0}=0 .
$$

Замечая, что $\rho$ - монотонно возрастающая функция, имеем

$$
\rho\left(v_{-}(r)\right) \leqslant \rho\left(\int_{0}^{r} q(\xi) d \xi\right)
$$

где $v_{-}(r)=\max \left(r q_{-}, r q_{+}+1-q_{-}\right)$, a

$$
q_{-} \stackrel{(1.16)}{=} \inf q, \quad q_{+} \stackrel{(1.16)}{=} \sup q
$$

Учитывая (1.43), получаем, что условие (1.22) будет выполнено, если

$$
0 \leqslant q^{-2}(r) q^{\prime}(r) \leqslant \rho\left(v_{-}(r)\right) .
$$

ЗАмечАниЕ 1.4. Выбрав вместо вспомогательной функции $D_{+}$решение задачи

$$
\left.\Delta D^{*}\right|_{Q}=0,\left.\quad D^{*}\right|_{v=0}=0,\left.\quad D^{*}\right|_{u=M}=N_{+},\left.\quad D^{*}\right|_{v=1}=\pi,\left.\quad D^{*}\right|_{u=0}=\frac{\pi}{2},
$$

получаем без каких-либо ограничений на функцию $N$ другое достаточное условие несуществования $\gamma_{2}$, а именно:

$$
\frac{\partial D^{*}}{\partial u}(0, v) \leqslant-\frac{R^{\prime \prime}}{R^{\prime}}(v) \leqslant \frac{\partial C_{-}}{\partial u}(0, v) \quad \forall v \in(0,1) .
$$

Это условие, очевидно, непротиворечиво при любом $M$, если $N_{+} \leqslant N_{-}+\pi / 2$, а при $M \gg 1$ и в случае $N_{+}>N_{-}+\pi / 2$. Следует, однако, отметить, что условие (1.48), в отличие от условия (1.47), не включает случай $q=$ const. 
1.9. Этот пункт (содержащий два подпункта) является основным в 1 . Здесь представлены доказательства теорем 1.1 и 1.7 существования искомой свободной границы $\gamma_{1}$. Основой доказательств является конструкция, позволяющая найти некоторый мультипараметр $\sigma=\left(\sigma_{1}, \ldots, \sigma_{m}\right) \in \mathscr{D}_{m} \Subset \mathbb{R}^{m}$, который соответствует геометрии полигонального контура $\Gamma_{m}$, аппроксимирующего заданный криволинейный контур Г. Идея полигональной аппроксимации, первоначально высказанная Н. Е. Жуковским ${ }^{4}$, в некоторых случаях была реализована В.Н.Монаховым [50]. Криволинейный контур Г предварительно аппроксимируется полигональным $\Gamma_{m}$. Затем с помощью теории Лере-Шаудера устанавливается существование неподвижной точки $\sigma=\sigma^{\lambda}$ для гомотопически эквивалентных при $\lambda \in[0,1]$ отображений $F_{m}^{\lambda}: \mathscr{D}_{m} \ni \sigma \mapsto F_{m}^{\lambda}(\sigma) \in \mathbb{R}^{m}$. Они соответствуют (удовлетворяющей определенным геометрическим ограничениям) деформации контура $\Gamma_{m}$ в квадрат $|x|+|y|=1$, которому отвечает почти тождественное отображение $F_{m}^{0}$. Здесь важно сразу отметить следующее. В случае банахова пространства $X$ требуемая в теореме Лере-Шаудера компактность отображения $K^{\lambda}=I-F^{\lambda}: \mathscr{D} \rightarrow X$ эквивалентна тому, что $K^{\lambda}$ является равномерным пределом конечномерных отображений. При доказательстве теорем 1.1 и 1.7 также применяются конечномерные аппроксимации $K_{m}^{\lambda}=I-F_{m}^{\lambda}: \mathscr{D}_{m} \rightarrow \mathbb{R}^{m}$, но они в нашем методе напрямую связаны с геометрическими характеристиками контура Г (с его самонепересекаемостью), а не с требованием равномерной сходимости $K_{m}^{\lambda} \rightarrow K^{\lambda}$. А это позволяет снять обременительное ограничение компактности отображения $K^{\lambda}: \mathscr{D} \rightarrow X$, которое при непосредственном применении теоремы Лере-Шаудера в пространстве $X$ гёльдеровых функций (возникающих в случае криволинейного контура Г) приводит к очень жестким ограничениям на геометрию кривой $Г$.

Отметим еще одну особенность функционально-геометрического метода, которая позволила сравнительно легко завершить доказательство теорем существования при переходе от полигональных контуров $\Gamma_{m}$ к спрямляемому контуру Г. Она опять-таки связана с геометрией - с геометрически очевидной оценкой $\left|z_{m}(w)\right| \leqslant C$ при всех $m \geqslant 1$ для функции $z_{m}$, гомеоморфно отображающей границу прямоугольника $Q \stackrel{(1.33)}{=}\{0<u<M, 0<v<1\}$ на $\Gamma_{m} \rightarrow \Gamma$. Эта оценка влечет существование такой подпоследовательности $z_{m_{k}}$, которая равномерно сходится на каждом компакте $K \subset Q$ к искомой аналитической функции

$$
Q \ni w \mapsto z(w)=z(0)+\int_{0}^{w} \exp (A+i B) d w \in \Omega
$$

Сказанное иллюстрирует общий принцип функционально-геометрического метода: сочетание функциональных и геометрических рассмотрений при анализе задачи Римана-Гильберта, соответствующей задачам со свободной границей для гармонических функций.

1.9.1. В этом подпункте приведено дОКАЗАТЕЛЬСТвО ТЕОРЕмЫ 1.1. Начнем с базового случая, т. е. когда Г- полигональный контур. В этом случае

\footnotetext{
${ }^{4}$ в заключительных словах его работы [49] о перспективе решения задачи обтекания плоского криволинейного препятствия: “Может быть, эта задача могла бы быть разрешена, как предельный случай задачи об ударе (т. е. обтекании - А. Д.) потока на многогранный контур”.
} 
функция $\varphi$, фигурирующая в задаче (1.39), задается следующей формулой:

$$
\varphi(v)=\sum_{j=1}^{m+1} N_{j} 1_{\left(\sigma_{j-1}, \sigma_{j}\right)}(v) .
$$

Здесь $N_{j}$ - угол между осью $x$ и внешней нормалью к $j$-й стороне полигона $\Gamma \cap \mathbb{R}_{++}^{2}$, функция $[0,1] \ni v \mapsto 1_{\left(\sigma_{j-1}, \sigma_{j}\right)}(v)$ равна 1 при $\sigma_{j-1}<v<\sigma_{j}$ и нулю вне этого интервала, а мультипараметр $\sigma=\left(\sigma_{1}, \ldots, \sigma_{m}\right)$ удовлетворяет условию

$$
\sigma=\left(\sigma_{1}, \ldots, \sigma_{m}\right) \in \mathfrak{D} \stackrel{\text { def }}{=}\left\{\sigma \in \mathbb{R}^{m} \mid 0=\sigma_{0}<\sigma_{1}<\cdots<\sigma_{m}<\sigma_{m+1}=1\right\},
$$

причем

$$
l_{j}=\int_{\sigma_{j-1}}^{\sigma_{j}} e^{A(M, v)} d v
$$

где $l_{j}$ - длина $j$-го сегмента ломаной $\Gamma \cap \mathbb{R}_{++}^{2}$. Наша первая цель - доказать, что в рассматриваемом здесь случае (т. е. при функции $\varphi$, заданной формулой (1.50), и $\chi=\pi / 2, \psi=-R^{\prime \prime} / R^{\prime}$ ) задача (1.39) имеет ограниченное решение $B: Q \rightarrow \mathbb{R}$. Доказательство проведем в два этапа.

Эman 1. Для мультипараметра $\sigma=\left(\sigma_{1}, \ldots, \sigma_{m}\right)$, удовлетворяющего условию (1.51), обозначим через $B^{\sigma}$ ограниченное решение задачи $(1.39)$, а через $A^{\sigma}$ обозначим гармонически-сопряженную к нему функцию (определенную с точностью до аддитивной постоянной).

ПреДЛОжЕНИЕ 1.2. Для любого $\sigma \in \mathfrak{D}$ отображение

$$
Q \ni w \mapsto z^{\sigma}(w)=z^{\sigma}(0)+\int_{0}^{w} \exp \left(A^{\sigma}+i B^{\sigma}\right) d w
$$

голоморбно в $Q$ и непрерывно в $\bar{Q}$.

ДокАзАтельство. Не очевидна лишь непрерывность функции $w \mapsto z(w)$ в точках вида $w=M+i v$, где $v \in[0,1]$. Для простоты записи опустим индекс $\sigma$ в обозначениях функций $A^{\sigma}$ и $B^{\sigma}$. Положим $w_{1}=M+i v_{1}$ и $w_{2}=u_{2}+i v_{2}$, где $u_{2} \leqslant$. Имеем

$$
\left|z\left(w_{1}\right)-z\left(w_{2}\right)\right| \leqslant\left|\int_{v_{2}}^{v_{1}} e^{A\left(u_{2}, v\right)} d v\right|+\left|\int_{u_{2}}^{M} e^{A\left(u, v_{1}\right)} d u\right| .
$$

Заметим, что

$$
e^{A(u, v)}=e^{A(0, v)} \exp \left\{\int_{0}^{u} \frac{\partial \beta}{\partial v}(\xi, v) d \xi\right\} \exp \left\{\int_{0}^{u} \frac{\partial b}{\partial v}(\xi, v) d \xi\right\},
$$

где $\beta$ и $b$ - решения следующих задач:

$$
\left.\Delta \beta\right|_{Q}=0,\left.\quad \beta\right|_{v=0}=0,\left.\quad \beta\right|_{u=M}=\varphi,\left.\quad \beta\right|_{v=1}=\frac{\pi}{2},\left.\quad \frac{\partial \beta}{\partial u}\right|_{u=0}=0
$$

и

$$
\left.\Delta b\right|_{Q}=0,\left.\quad b\right|_{v=0}=0,\left.\quad b\right|_{u=M}=0,\left.\quad b\right|_{v=1}=0,\left.\quad \frac{\partial b}{\partial u}\right|_{u=0}=\psi
$$


Полагая $N_{0}=0$ и $N_{m+2}=\pi / 2$, получаем

$$
e^{A(u, v)}=\frac{R^{\prime}(v)}{R^{\prime}(0)} \exp \{A(0,0)\} E(u, v) \exp \left\{\int_{0}^{u} \frac{\partial b}{\partial v}(\xi, v) d \xi\right\}
$$

где функция $E$ определена в замечании 1.1. В силу леммы 1.2 , интеграл от $\partial b / \partial v$ равномерно ограничен по $v \in(0,1)$, а $R^{\prime}(v) \leqslant 1 / q_{-}$(см. (1.42), (1.46)). Поэтому

$$
\left|\int_{v_{2}}^{v} e^{A(u, \eta)} d \eta\right| \leqslant C\left|\int_{v_{2}}^{v} E(u, \eta) d \eta\right| \text { и } \quad \int_{u}^{M} e^{A(\xi, v)} d \xi \mid \leqslant C \int_{u}^{M} E(\xi, v) d \xi,
$$

где $C$ не зависит от $u \in[0, M]$ и $v \in[0,1]$. В результате, учитывая замечание 1.1 , получаем непрерывность функции $z$ в точке $w=M+i v$. Предложение 1.2 доказано.

Эman 2. Приведенное доказательство предложения 1.2 включает также проверку того, что функция $\exp \left\{A^{\sigma}(M, \cdot)\right\}$ интегрируема на $[0,1]$. Используя это, будем в дальнейшем считать, что функция $A=A^{\sigma}$ (определенная до сих пор с точностью до аддитивной константы) удовлетворяет условию

$$
\int_{0}^{1} \exp \{A(M, v)\} d v=\left|\Gamma^{0}\right|, \quad \text { где } \Gamma^{0}=\Gamma \cap \overline{\mathbb{R}_{++}^{2}} .
$$

Зафиксируем также точку $z(0)$, положив

$$
z(0)=\int_{0}^{1} e^{A(0, v)} \sin B(0, v) d v .
$$

Легко видеть, что в этом случае ломаная

$$
\Gamma_{\sigma}^{0}=\left\{z \in \mathbb{C} \mid z=z^{\sigma}(M, v), v \in[0,1]\right\},
$$

определяемая отображением (1.53), лежит в правой полуплоскости, а ее концы находятся на осях координат. Сегменты ломаной $\Gamma_{\sigma}^{0}$ имеют (по построению) те же углы наклона к оси $x$, что и соответствующие сегменты заданной ломаной $\Gamma^{0}=\Gamma \cap \overline{\mathbb{R}_{++}^{2}}$. Однако длина

$$
L_{j}(\sigma)=\left|z\left(M+i \sigma_{j}\right)-z\left(M+i \sigma_{j-1}\right)\right|=\int_{\sigma_{j-1}}^{\sigma_{j}} e^{A(M, v)} d v
$$

$j$-го сегмента ломаной $\Gamma_{\sigma}^{0}$, вообще говоря, отлична от длины $l_{j}$ соответствующего, т. е. $j$-го, сегмента заданной ломаной $\Gamma^{0}$. Совпадение этих ломаных эквивалентно равенству длин соответствующих сегментов. Последнее означает, что мультипараметр $\sigma \in \mathfrak{D} \subset \mathbb{R}^{m}$ является решением уравнения

$$
F(\sigma)=\sigma, \quad \text { где } F: \mathfrak{D} \ni \sigma \mapsto F(\sigma)=\left(F_{1}(\sigma), \ldots, F_{m}(\sigma)\right) \in \mathbb{R}^{m},
$$

a $F_{j}(\sigma)=\sigma_{j} L_{j}(\sigma) / l_{j}$. Существование искомой неподвижной точки $\sigma$ отображения $F$ вытекает из нижеследующих четырех лемм и теоремы Лере-Шаудера (см., например, [51], [52]). 
Лемма 1.4. Существует семейство самонепересекающихся ломаных $\Gamma^{\lambda}$, состоящих из $m+1$ сегментов $\left\{l_{j}^{\lambda}, N_{j}^{\lambda}\right\}_{j=1, \ldots, m+1}$, имеющих длину $l_{j}^{\lambda}$ и наклоненных к оси $x$ под углом $N_{j}^{\lambda}+\pi / 2$, которое обладает следующими свойствами:

1) $\Gamma^{0}=\Gamma \cap \overline{\mathbb{R}_{++}^{2}}$;

2) $\Gamma^{\lambda} \cap \partial\left(\overline{\mathbb{R}_{++}^{2}}\right)=\partial \Gamma^{\lambda}$ для любого $\lambda \in[0,1]$;

3) $N_{j}^{1}=\pi / 4, l_{j}^{1}=1 /(m+1)$;

4) функиии $\lambda \mapsto l_{k}^{\lambda}$ и $\lambda \mapsto N_{k}^{\lambda}$ достаточно гладкие;

5) существует $\delta>0$ такое, что

$$
l_{k}^{\lambda}>\delta>0 \quad \forall k, \quad \operatorname{dist}\left(\Gamma^{\lambda}, 0\right)>\delta,
$$

где $\operatorname{dist}\left(\Gamma^{\lambda}, 0\right)$ - расстояние между $\Gamma^{\lambda}$ и точкой $z=0$.

При $\lambda \in[0,1 / 2]$ построение указанного семейства $\Gamma^{\lambda}$ ясно из представленного здесь рис. 8. Далее делается подходящая гомотетия.

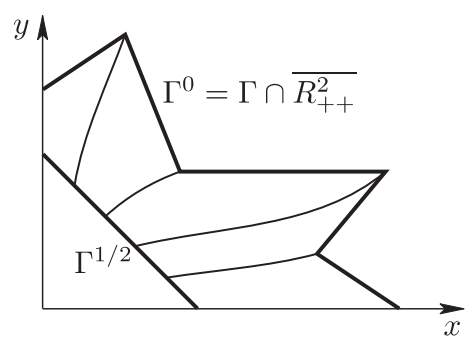

Рис. 8

Следуя В.Н. Монахову (см. [50; гл. III, §3]), рассмотрим семейство отображений

$$
[0,1] \ni \lambda \mapsto F^{\lambda}: \mathfrak{D} \ni \sigma \mapsto F^{\lambda}(\sigma)=\left(F_{1}^{\lambda}(\sigma), \ldots, F_{m}^{\lambda}(\sigma)\right) \in \mathbb{R}^{m},
$$

где $F_{j}^{\lambda}(\sigma)=\sigma_{j} L_{j}^{\lambda}(\sigma) / l_{j}^{\lambda}$, а $L_{j}^{\lambda}(\sigma)$ определяется аналогично (1.58).

Лемма 1.5. Существует такое $\varepsilon>0$, что для любого $\lambda \in[0,1]$ всякое решение уравнения $F^{\lambda}(\sigma)=\sigma$ удовлетворяет условию: $\sigma \in \mathscr{D}$, где

$$
\mathscr{D}=\mathfrak{D}_{\varepsilon} \stackrel{\text { def }}{=}\left\{\sigma \stackrel{(1.51)}{\in} \mathfrak{D} \mid \sigma_{j}-\sigma_{j+1}>\varepsilon, j=1, \ldots, m+1\right\} .
$$

ДокАЗАТЕЛЬСтво. Предположим противное. Тогда для любого $n \geqslant 1$ существуют $\lambda_{n} \in[0,1]$ и $\sigma^{n} \in \mathfrak{D} \backslash \mathfrak{D}_{1 / n}$ такие, что

$$
F^{\lambda_{n}}\left(\sigma^{n}\right)=\sigma^{n}, \quad \text { т. е. } L_{j}^{\lambda_{n}}\left(\sigma^{n}\right)=l_{j}^{\lambda_{n}} \forall j=1, \ldots, m .
$$

Согласно (1.58),

$$
L_{j}^{\lambda}(\sigma)=\left|\Gamma^{\lambda}\right|\left(\int_{\sigma_{j-1}}^{\sigma_{j}} H^{\sigma, \lambda}(M, v) d v\right)\left(\int_{0}^{1} H^{\sigma, \lambda}(M, v) d v\right)^{-1}
$$


где зависимость функции

$$
H^{\sigma, \lambda}(u, v)=R^{\prime}(0) \frac{e^{A(u, v)}}{e^{A(0,0)}} \stackrel{(1.55)}{=} R^{\prime}(v) E(u, v) \exp \left\{\int_{0}^{u} \frac{\partial b}{\partial v}(\xi, v) d \xi\right\}
$$

от $\lambda$ и $\sigma$ иногда для упрощения записи не будет явно обозначаться.

Можно считать, что последовательности $\left\{\sigma^{n}\right\}_{n \geqslant 1}$ и $\left\{\lambda_{n}\right\}_{n \geqslant 1}$ фундаментальны. Рассмотрим поведение интеграла $\int_{0}^{1} H(M, v) d v$ при $n \rightarrow \infty$. Так как $\sigma^{n} \notin \mathfrak{D}_{1 / n}$, то существует $j=j(n)$ такое, что $0<\sigma_{j}^{n}-\sigma_{j-1}^{n} \leqslant 1 / n$. Имеются две возможности: либо интеграл остается ограниченным, либо он стремится к бесконечности. В первом случае $L_{j}^{\lambda_{n}}\left(\sigma^{n}\right) \rightarrow 0$, так как $\left|\Gamma^{\lambda_{n}}\right| \leqslant$ const. Отсюда следует, в силу (1.62), (1.63), что $l_{j}^{\lambda_{n}} \rightarrow 0$, а это невозможно по лемме 1.4. Во втором случае рассмотрим кривую $\gamma^{n}=\left\{z_{n}(i v), v \in[0,1]\right\}$, где отображение $z_{n}$ построено для $A=A^{\sigma^{n}, \lambda_{n}}$ и $B=B^{\sigma^{n}, \lambda_{n}}$. Длина этой кривой определяется формулой

$$
\left|\gamma^{n}\right|=\int_{0}^{1} e^{A_{0}} d v=\left|\Gamma^{\lambda_{n}}\right|\left(\int_{0}^{1} H(M, v) d v\right)^{-1}
$$

из которой следует, что $\left|\gamma^{n}\right| \rightarrow 0$. Таким образом, $\gamma^{n}$ стягивается в начало координат. Поэтому мы получим нужное нам противоречие с условием (1.60), если покажем, что

$$
\operatorname{dist}\left(\Gamma^{\lambda_{n}}, \gamma^{n}\right) \leqslant\left|\Gamma^{\lambda_{n}}\right|\left(\int_{0}^{1} H(M, v) d v\right)^{-1} \inf _{v \in[0,1]} \int_{0}^{M} H(u, v) d u,
$$

ибо $\inf _{v \in[0,1]} \int_{0}^{M} H(u, v) d u \leqslant \int_{0}^{M} H\left(u, v_{*}\right) d u \leqslant$ const при $v_{*} \in[0,1] \backslash \overline{\bigcup_{j, n} \sigma_{j}^{n}}$. Что же касается неравенства (1.66), то вот его доказательство:

$$
\begin{aligned}
& \inf _{v \in[0,1]} \inf _{\eta \in[0,1]}\left|z_{n}(M+i v)-z_{n}(i \eta)\right| \leqslant \inf _{v \in[0,1]}\left|\int_{i v}^{M+i v} e^{A+i B} d w\right| \\
& \quad \leqslant \inf _{v \in[0,1]} \frac{e^{A(0,0)}}{R^{\prime}(0)} \int_{0}^{M} H(u, v) d u \\
& \quad \leqslant\left|\Gamma^{\lambda_{n}}\right|\left(\int_{0}^{1} H(M, v) d v\right)^{-1} \inf _{v \in[0,1]} \int_{0}^{M} H(u, v) d u .
\end{aligned}
$$

Лемма 1.5 доказана.

ЛЕмма 1.6. Отображение $\mathfrak{D} \times[0,1] \ni(\sigma, \lambda) \mapsto F^{\lambda}(\sigma)$ непреръвно.

ДокАзАТЕЛЬСтво. В силу формулы (1.63) и того факта, что

$$
\int_{0}^{1} H^{(\sigma, \lambda)}(M, v) d v \stackrel{(1.64)}{>} 0 \quad \forall(\sigma, \lambda) \in \mathfrak{D} \times[0,1],
$$

достаточно проверить непрерывность двух отображений

$$
(\sigma, \lambda) \mapsto \int_{0}^{1} H^{\sigma, \lambda}(M, v) d v, \quad(\sigma, \lambda) \mapsto \int_{0}^{1} 1_{\left(\sigma_{j-1}, \sigma_{j}\right)}(v) H^{\sigma, \lambda}(M, v) d v,
$$


определенных для $(\sigma, \lambda) \in \mathfrak{D} \times[0,1]$. Пусть $\left(\sigma^{n}, \lambda_{n}\right) \rightarrow\left(\sigma^{*}, \lambda_{*}\right)$. Имеем

$$
\sigma^{*}=\left\{\sigma_{1}^{*}, \ldots, \sigma_{m}^{*}\right\}, \quad \text { где } 0=\sigma_{0}^{*} \leqslant \sigma_{1}^{*} \leqslant \cdots \leqslant \sigma_{m}^{*} \leqslant \sigma_{m+1}^{*}=1 .
$$

Заметим, что для любого $\delta>0$ существует такое открытое множество $C_{\delta} \subset \mathbb{R}$, меры меньшей $\delta$, содержащее все точки $\sigma_{j}^{*}$, что вне $C_{\delta}$ последовательность функций $H^{\sigma^{n}, \lambda_{n}}(M, \cdot)$ сходится поточечно при $n \rightarrow \infty$ к функции $H^{\sigma^{*}, \lambda_{*}}(M, \cdot)$, которая интегрируема, согласно замечанию 1.1. Каждый элемент этой последовательности функций ограничен в $L^{p}(0,1)$ с некоторым $p>1$. Остается сослаться на теорему Витали (ср. лемму 1.1), и непрерывность отображений (1.67), а вместе с ней и лемма 1.6 доказаны.

Лемма 1.7. Уравнение $F^{1}(\sigma)=\sigma$ имеет единственное решение $\hat{\sigma} \in \mathscr{D}$, в окрестности которого отображение $I-F^{1}$ биективно.

ДокАЗАТЕЛЬСтво. При $\lambda=1$ функции $A=A^{1}$ и $B=B^{1}$ не зависят от $\sigma=\left(\sigma_{1}, \ldots, \sigma_{m}\right)$, так как (согласно лемме 1.4$) N_{j}^{1}=\pi / 4$ для каждого $j=1, \ldots, m+1$. А так как $l_{j}^{1}=1 /(m+1)$, то искомые компоненты неподвижной точки $\hat{\sigma}=\left(\hat{\sigma}_{1}, \ldots, \hat{\sigma}_{m}\right)$ отображения $F^{1}$ определятся последовательно из следующей системы уравнений:

$$
l_{j}^{1}=\frac{1}{m+1}=\int_{\hat{\sigma}_{j-1}}^{\hat{\sigma}_{j}} e^{A(M, v)} d v, \quad j=1, \ldots, m, \quad \text { c } \hat{\sigma}_{0}=0, \hat{\sigma}_{m+1}=1 .
$$

Якобиан $J(\sigma)$ отображения $I-F^{1}(\sigma)$ легко считается. Имеем $F_{j}^{1}(\sigma) \stackrel{(1.59)}{=}$ $\sigma_{j} \int_{\sigma_{j-1}}^{\sigma_{j}} e^{A(M, v)} d v / l_{j}^{1}$. При этом функция $(0,1) \ni v \mapsto A(M, v)$ аналитична, ибо

$$
B(M, v)=\text { const при } 0<v<\left.1 \Longrightarrow \frac{\partial A(u, v)}{\partial u}\right|_{u=M}=0 .
$$

Имеем $\frac{\partial F_{j}(\sigma)}{\partial \sigma_{k}}=0$ для всех $k>j$, поэтому $J(\sigma)=\prod_{j=1}^{m}\left(1-\frac{\partial F_{j}(\sigma)}{\partial \sigma_{j}}\right)$. Ясно, что

$$
\frac{\partial F_{j}(\sigma)}{\partial \sigma_{j}}=\frac{1}{l_{j}^{1}} \int_{\sigma_{j-1}}^{\sigma_{j}} e^{A(M, v)} d v+\frac{\sigma_{j}}{l_{j}^{1}} e^{A\left(M, \sigma_{j}\right)} .
$$

Учитывая (1.68), получаем

$$
J(\hat{\sigma})=\prod_{j=1}^{m}\left(-\frac{\hat{\sigma}_{j}}{l_{j}^{1}} e^{A\left(M, \hat{\sigma}_{j}\right)}\right) \neq 0 .
$$

Остается отметить непрерывность якобиана $J$ (что является следствием его аналитичности). Лемма 1.7 доказана.

Подведем первый итог. Доказаны леммы 1.4-1.7. Поэтому, в силу теоремы Лере-Шаудера, задача (1.39) в рассматриваемом здесь случае (т. е. при функции $\varphi$, заданной формулой $(1.50)$, и $\left.\chi=\pi / 2, \psi=-R^{\prime \prime} / R^{\prime}\right)$ имеет ограниченное решение $B$. Более того, приведенное доказательство показывает, что задача (1.39) разрешима и в случае $\left|\delta N_{j}\right|<1$ (ср. замечание 1.1). Это условие 
эквивалентно тому, что полигональный контур $\Gamma^{0}$ самонепересекается. Однако, согласно теореме 1.4 (см. также рис. 3) условие $\left|\delta N_{j}\right|<1$ недостаточно для справедливости теоремы 1.1. Осознать причины этого позволяют следующие две леммы, которые устанавливают соответственно однолистность отображения $z: Q \rightarrow \Omega$ и выполнение неравенства (1.40).

ЛЕмма 1.8. Пусть $\sigma$ - неподвижная точка отображения (1.61), и пусть

$$
0 \leqslant N(s) \leqslant \pi \quad \text { nрu } P_{s} \in \Gamma \cap \mathbb{R}_{++}^{2} .
$$

Тогда функиия z, определенная формулой (1.49), биективно отображает прямоугольник $Q$ на односвлзную область $\omega \subset\left\{(x, y) \in \mathbb{R}^{2} \mid x>0\right\}$, граница которой есть замкнутая кусочно гладкая кривая $\partial \omega$, состоящая из отрезка $[z(0), z(M)] \subset \mathbb{R}_{x}$, ломаной $\Gamma \cap \overline{\mathbb{R}_{++}^{2}}$, отрезка $[z(i), z(M+i)] \subset \mathbb{R}_{y}$ и кривой

$$
[0,1] \ni v \mapsto z(i v)=z(0)+i \int_{0}^{i v} e^{A(0, \eta)+i B(0, \eta)} d \eta
$$

соединяющей точки z(0) и $z(i)$.

ДокАЗАТЕЛЬСтво. В силу принципа максимума для гармонических функций, условие (1.69) влечет неравенства

$$
0 \leqslant B(0, v) \leqslant \pi \quad \forall v \in[0,1]
$$

где $B$ - решение задачи (1.39) (и потому $B(0,0)=0$, а $B(0,1)=\pi / 2$ ).

Из предложения 1.2 следует, что функция (1.49) гомеоморфно и с сохранением ориентации отображает на $\partial Q$ вышеописанную кривую $\partial \omega$. Остается показать, что эта кривая самонепересекается. Для этого, ввиду (1.71), достаточно прийти к противоречию с таким предположением:

$$
\text { кривая (1.70) и ломаная } \Gamma \cap \overline{\mathbb{R}_{++}^{2}} \text { имеют общую точку. }
$$

Для того чтобы опровергнуть это предположение, покажем сначала, что

$$
z(Q) \subset \mathbb{C} \backslash \bar{\Lambda}
$$

где $\Lambda$ - неограниченная связная компонента множества $\mathbb{C} \backslash z(\partial Q)$.

Достаточно проверить, что $z(Q) \subset \mathbb{C} \backslash \Lambda$, ибо множество $z(Q)$ открыто. Предположим вопреки (1.73), что существует точка $z_{*} \in z(Q) \cap \Lambda$. Поскольку область $z(Q)$ ограничена, то кривая, лежащая в $\Lambda$ и соединяющая $z_{*}$ и $\infty$, пересекает $\partial z(Q)$. Таким образом, существует точка $y_{*} \in \partial z(Q) \cap \Lambda$. Но $y_{*} \neq z(\partial Q)$, так как $\Lambda \cap z(\partial Q)=\varnothing$. Мы получили противоречие с включением $\partial z(Q) \subset z(\partial Q)$, верным для любого открытого отображения (и, в частности, для голоморфной функции $z)$. Другими словами, мы доказали включение (1.73). Покажем, что из него следует такой факт:

$$
\text { множество } \mathbb{C} \backslash \bar{\Lambda} \text { связно. }
$$

В самом деле, в противном случае найдутся две связные компоненты множества $\mathbb{C} \backslash \bar{\Lambda}$ (ограниченные в силу ограниченности $\mathbb{C} \backslash \bar{\Lambda}$ ), одна из которых, согласно (1.73), содержит множество $z(Q)$ (ввиду его связности), а граница другой содержит точку $p \notin \overline{z(Q)}=z(\bar{Q})$, что противоречит следующему:

$$
p \in \partial(\mathbb{C} \backslash \bar{\Lambda}) \subset z(\partial Q) \subset z(\bar{Q}) .
$$


Теперь легко установить требуемое для доказательства леммы опровержение предположения (1.72). Действительно, как мы сейчас увидим, справедлива импликация

$$
(1.72) \Longrightarrow \text { множество } \mathbb{C} \backslash \bar{\Lambda} \text { не связно, }
$$

которая противоречит уже установленному факту (1.74).

Докажем (1.75). Рассмотрим встречное движение вдоль кривой (1.70) двух точек $P_{0}(v)=z(0, v)$ и $P_{1}(v)=z(0,1-v)$ при изменении параметра $v$ от значения $v=0$ до $v=1$. Согласно предположению $(1.72)$, каждая точка $P_{j}$ при некотором $v_{j} \in(0,1)$ впервые достигнет ломаную $\Gamma \cap \overline{\mathbb{R}_{++}^{2}}$. В силу $(1.71)$, у ломаной $\Gamma \cap \overline{\mathbb{R}_{++}^{2}}$ и кривой $(1.70)$ нет общих точек, лежащих правее $P_{0}\left(v_{0}\right)$ или левее $P_{1}\left(v_{1}\right)$. Поэтому получаем, что два непересекающихся множества $M_{0}$ и $M_{1}$ (см. рис. 9) должны принадлежать множеству $\mathbb{C} \backslash \bar{\Lambda}$. Тем самым, импликация (1.75) доказана. Лемма 1.8 доказана.

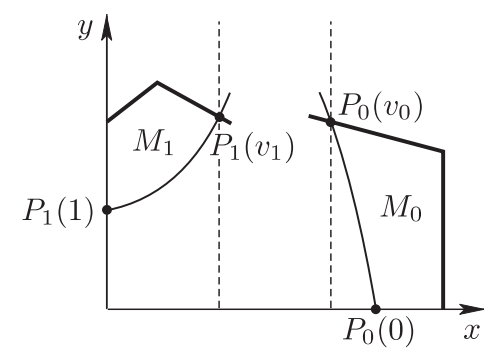

Рис. 9

ЛЕмма 1.9. Неравенство (1.40) справедливо, если выполнено условие (1.19). ДокАЗАтЕЛьСтво. Из (1.19)-(1.21), (1.44)-(1.45) и леммы 1.3 вытекает, что

$$
0=C_{-}(0, v) \leqslant B_{-}(0, v) \leqslant B(0, v) \leqslant B_{+}(0, v) \leqslant C_{+}(u, 0)=\frac{\pi}{2} .
$$

Таким образом, $0 \leqslant B(0, v) \leqslant \pi / 2$ при $v \in[0,1]$, причем $B_{+}(0, v)<\pi / 2$ при $v \sim 0$, так как $B_{+}(0, \cdot) \in C[0,1]$. Поэтому

$$
\int_{0}^{v} R^{\prime}(\eta) \cos B(0, \eta) d \eta \geqslant \int_{0}^{v} R^{\prime}(\eta) \cos B_{+}(0, \eta) d \eta>0 \quad \forall v \in(0,1] .
$$

Лемма 1.9 доказана.

Второй итог: теорема 1.1 доказана для полигональных контуров.

Докажем ее теперь для случая спрямляемого контура $\Gamma$. Пусть $\Gamma_{m}-$ семейство таких симметричных относительно координатных осей полигональных контуров, вписанных в $\Gamma$, что длины всех $m+1$ сегментов ломаной $\Gamma_{m} \cap \overline{\mathbb{R}_{++}^{2}}$ стремятся к нулю при $m \rightarrow \infty$. Пусть $A_{m}+i B_{m}-$ решение задачи (1.39) при $\varphi=N_{m} \circ s$, где $N_{m}$ - угловая функция контура $\Gamma_{m}$. Обозначим через $z_{m}$ функцию, определенную формулой (1.49). Функция $z_{m}$ биективно отображает $Q$ на область, описанную в лемме 1.8. По построению, $\Gamma_{m}$ преобразуется в $Г$ при $m \rightarrow \infty$. Поэтому существует такая константа $C$, что

$$
\left|z_{m}(w)\right| \leqslant C \quad \forall w \in Q, \quad \forall m \geqslant 1 .
$$


Следовательно, существует такая подпоследовательность $z_{m_{k}}$, которая равномерно сходится на каждом компакте $K \subset Q$ к гармонической в $Q$ функции.

Лемма 1.10. Функция $z=\lim _{k \rightarrow \infty} z_{m_{k}}: Q \rightarrow \mathbb{C}$ отлична от константы.

ДокАЗАТЕЛЬСтво. Достаточно прийти к противоречию предположением

$$
\int_{0}^{1} \exp \left\{A_{m}(0, v)\right\} d v \rightarrow 0 \quad \text { при } m \rightarrow \infty,
$$

которое, в силу $(1.65)$, означает, что $\gamma_{m}$ стягивается в начало координат и

$$
\int_{0}^{1} H_{m}(M, v) d v \rightarrow \infty \quad \text { при } m \rightarrow \infty .
$$

Отсюда и из (1.66) следует требуемое противоречие, поскольку, в силу (1.64),

$$
\inf _{v \in[0,1]} \int_{0}^{M} H_{m}(u, v) d u \leqslant C \inf _{v \in[0,1]} \int_{0}^{M} E_{m}(u, v) d u \leqslant C_{*}<\infty \quad \forall m .
$$

Лемма 1.10 доказана.

Подведем окончателъный итог. Согласно известному свойству о равномерной сходимости (на каждом компакте $K \subset Q$ ) однолистных отображений (см., например, в [53; гл. V, § 1, предложение 2.2]), из леммы 1.10 следует, что отображение $z: Q \rightarrow \mathbb{C}$ есть биекция на свой образ $\omega=z(Q)$, являющийся, очевидно, областью со свойствами, описанными в леммах 1.8 и 1.9. Теорема 1.1 полностью доказана.

1.9.2. Выделим теперь основные моменты доказательства теоремы 1.7.

$1^{\circ}$. Вывод необходимых условий разрешимости. Если исходная задача разрешима, то, в соответствии с геометрическими данными задачи, существует аналитическая функция

$$
w=u+i v: \Omega \rightarrow Q=\{(u, v) \in\{0<u<M, v \in \mathbb{R} / \mathbb{Z}\},
$$

а определенная в $Q$ аналитическая функция $A+i B=\ln (d z / d w)$ является решением следующей задачи Римана-Гильберта:

$$
B_{u}(0, v)=0, \quad B(M, v)=N(s(v)), \quad s(v)=\int_{0}^{v} \exp \{A(M, \eta)\} d \eta .
$$

$2^{\circ}$. Разрешимость нелинейной задачи Римана-Гильберта. Докажем существование решения задачи (1.78), предположив сначала, что Г - полигональный контур $\left\{N_{j}, l_{j}\right\}_{j=1}^{m}$, где $N_{j}$ есть угол между осью $x$ и внешней нормалью к $j$-му сегменту $\Gamma_{j}$ кривой $\Gamma, l_{j}=\left|\Gamma_{j}\right|$. Пусть $[0,1] \ni \lambda \mapsto \Gamma^{\lambda}=\left\{N_{j}^{\lambda}, l_{j}^{\lambda}\right\}_{j=1}^{m}$ есть гомотопия $\Gamma^{0}=\Gamma$ и $\Gamma^{1}=\{|x|+|y|=1\}$. Можно считать, что $\Gamma^{\lambda}$ выпукла, $\left|\Gamma^{\lambda}\right| \leqslant$ const, $l_{j}^{\lambda}$ и $N_{j}^{\lambda}$ гладки по $\lambda$ и, более того, $l_{j}^{\lambda} \geqslant \delta>0$ для любых $j$ и $\lambda$. Пусть $\sigma=\left(\sigma_{1}, \ldots, \sigma_{m}\right)$, причем $0=\sigma_{0}<\sigma_{1}<\cdots<\sigma_{m}<\sigma_{m+1}=1$. Положим $\Phi(v)=N_{j}^{\lambda}$, если $v \in\left(\sigma_{j-1}, \sigma_{j}\right)$. Пусть $B(u, v)=C(u, v)+2 \pi v$, где $C$ гармонична в цилиндре $(0, M) \times \mathbb{R} / \mathbb{Z}$, а $B(M, v)=\Phi(v), B_{u}(0, v)=0$. Пусть $A$ 
гармонически-сопряжена с $B$. Можно проверить, что $A(M, \cdot) \in L^{1}(0,1)$ и что аналитическая функция

$$
z: Q \ni w \mapsto z(w)=z(0)+\int_{0}^{w} \exp \{A+i B\} d w
$$

непрерывна в $\bar{Q}$. Множество $\{z(w) \in \mathbb{C} \mid w=(0, v), v \in[0,1]\}$ есть замкнутая полигональная кривая $\left\{N_{j}^{\lambda}, L_{j}^{\lambda}(\sigma)\right\}_{j=1}^{m}$, где $L_{j}^{\lambda}(\sigma)=\int_{\sigma_{j-1}}^{\sigma_{j}} \exp \{A(M, \eta)\} d \eta$. Нужно доказать, что отображение

$$
F^{\lambda}: \mathbb{R}^{m} \ni \sigma \longmapsto\left(F_{1}^{\lambda}(\sigma), \ldots, F_{m}^{\lambda}(\sigma)\right), \quad F_{j}^{\lambda}=\frac{\sigma_{j} L_{j}^{\lambda}(\sigma)}{l_{j}^{\lambda}},
$$

имеет неподвижную точку. Имеем: для любого $\delta>0$ существует $h>0$ такое, что любая неподвижная точка $\sigma=\sigma(\lambda)$ отображения $F^{\lambda}$ (если она существует) принадлежит $D_{h}=\left\{\sigma: \sigma_{j+1}-\sigma_{j}>h, j=0, \ldots, m\right\}$ для любого $\lambda \in[0,1]$. В самом деле, если некоторые параметры $\sigma_{j}, \ldots, \sigma_{j+k_{j}}$ сближаются, то имеются следующие две возможности.

1) Общий перепад (скачок) $N_{j+k_{j}+1}-N_{j}$ около $\sigma_{j}$ меньше $\pi$ для любого $j$. В этом случае функция $\exp \{A(M, v)\}$ (имеющая особенности $1 /\left|v-\sigma_{k}\right|^{\alpha_{k}}$, $\left.\alpha_{k}=\left(N_{k+1}-N_{k}\right) / \pi\right)$ равномерно интегрируема. Следовательно, $L_{j}^{\lambda}=l_{j}^{\lambda} \rightarrow 0$ (получаем противоречие с $l_{j}^{\lambda} \geqslant \delta>0$ ).

2) Общий перепад больше или равен $\pi$. Тогда, во-первых, $|\gamma| \rightarrow 0$, а во-вторых, найдутся две различные, т. е. отстоящие друг от друга на положительном расстоянии, точки $P_{1}^{*}$ и $P_{2}^{*}$ на $\Gamma^{\lambda}$, для которых $\operatorname{dist}\left(P_{k}^{*}, \gamma\right) \rightarrow 0$. (Точки $P_{k}^{*}$ соответствуют тем точкам $\left(M, v_{1}^{*}\right)$ и $\left(M, v_{2}^{*}\right)$ на границе прямоугольника $Q$, которые отстоят от сгущающихся точек разрыва функции $N$ на квалифицированно положительном расстоянии.) Сочетание двух указанных обстоятельств противоречит выпуклости кривой $\Gamma^{\lambda}$.

Итак, $\sigma \neq F^{\lambda}(\sigma)$ для любого $\lambda$, если $\sigma \in \partial D_{h}$. Можно проверить, что $(\sigma, \lambda) \mapsto F^{\lambda}(\sigma)$ есть непрерывное отображение на $\overline{D_{h}} \times[0,1]$, а уравнение $\sigma=$ $F^{1}(\sigma)$ имеет по крайней мере одно решение и $\operatorname{det}\left(\frac{\partial F^{1}}{\partial \sigma}\left(\sigma^{*}\right)-I\right)>0$ для любого $\sigma^{*} \equiv F^{1}\left(\sigma^{*}\right)$. Остается применить теорему Лере-Шаудера.

В случае спрямляемого контура строятся (как выше) отображения $z_{m}(w)$ для вписанных полигональных контуров, длины сторон которых стремятся к нулю. Так же как в (1.77), имеем $\left|z_{m}(w)\right| \leqslant$ const. Поэтому существует подпоследовательность $z_{m_{k}}$, которая равномерно сходится на каждом компакте $K \subset Q$ к гармонической в $Q$ функции $z=\lim _{k \rightarrow \infty} z_{m_{k}}$. Проверяется (также как в лемме 1.10), что $z \neq$ const. Поэтому, в силу упомянутого на стр. 35 предложения из [53], отображение $z: Q \rightarrow \mathbb{C}$ есть биекция на свой образ $\Omega=z(Q)$, внешняя граница замыкания которого есть исходная выпуклая кривая Г. Этим завершается доказательство теоремы 1.7.

$3^{\circ}$. Однолистность отображения (1.79). Достаточно доказать, что образ $\gamma$ отображения $[0,1] \ni v \mapsto z(0, v)$ есть самонепересекающаяся кривая. Это вытекает из ее выпуклости в силу того, что $B_{v}(0, v) \geqslant 0$. Последнее есть следствие принципа максимума и неубывания функции $v \mapsto B(M, v)$, что вытекает из выпуклости Г. 
1.10. Приведем доказательство теоремы 1.6 о существовании несимметричных конфигураций. По существу, все сводится к поиску мультипараметра $\sigma=\left(\sigma_{1}, \sigma_{2}, \sigma_{3}\right)$, компоненты которого удовлетворяют двум условиям:

$$
0=\sigma_{0}<\sigma_{1}<\sigma_{2}<1<\sigma_{3}<\sigma_{4}=2, \quad S_{1}=S_{4}>S_{2}=S_{3},
$$

где

$$
S_{j}\left(\sigma_{1}, \sigma_{2}, \sigma_{3}\right)=\int_{\sigma_{j-1}}^{\sigma_{j}} g(v) d v, \quad g(v) \stackrel{\text { def }}{=} \exp \left\{\int_{0}^{M} \frac{\partial B}{\partial v}(u, v) d u\right\},
$$

а $B=B^{\sigma}$ - гармоническая функция в $Q=\{0<u<M, 0<v<2\}$, удовлетворяющая (ср. (1.39)) таким граничным данным:

$$
B(u, 0)=0, \quad B(u, 1)=\pi, \quad B_{u}(0, v)=0, \quad B(M, v)=\varphi(v) .
$$

Здесь

$$
\varphi(v)= \begin{cases}\frac{\pi}{4} & \text { при } v \in\left(0, \sigma_{1}\right) \cup\left(\sigma_{2}, \sigma_{3}\right), \\ \frac{3 \pi}{4} & \text { при } v \in\left(\sigma_{1}, \sigma_{2}\right) \cup\left(\sigma_{3}, 2\right) .\end{cases}
$$

Ясно, что формулы (1.80) выражают геометрические соотношения между длинами $S_{j}$ последовательных четырех сегментов ломаной $\Gamma_{a} \cap \mathbb{R}_{+}^{2}$. Столь же очевидно соотношение

$$
S_{1}-S_{2}+S_{3}-S_{4}=\int_{0}^{\sigma_{4}} \cos B(0, v) d v .
$$

Как показано ниже, асимптотический анализ интегралов, представляющих функции $S_{j}$, позволяет доказать, что при достаточно малом $\rho \stackrel{\text { def }}{=} \sigma_{2}$ существуют такие параметры $\sigma_{1}=O\left(\rho^{2}\right)$ и $\sigma_{3}=\sigma_{3}(\rho)>\rho$, для которых выполнены соотношения (1.80). (В более общей ситуации показатель $\alpha$ в формуле $\sigma_{1}=O\left(\rho^{\alpha}\right)$ зависит от величины углов между сегментами контура $\Gamma_{a}$.) Малость параметра $\rho$ приводит к малости "перешейка" контура $\Gamma_{a}$, представленного на рис. 4. Это означает, что $S_{1}\left(\sigma_{1}(\rho), \rho, \sigma_{3}(\rho)\right)=\int_{0}^{\rho} \exp \left\{\int_{0}^{M} \frac{\partial B}{\partial v}(u, v) d u\right\} d v \rightarrow 1 / 2$ при $\rho \rightarrow 0$.

Проведем предварительные построения. Пусть

$$
\beta(u, v)=B(u, v)-b(u, v), \quad h(v)=\exp \left\{\int_{0}^{M} \frac{\partial \beta(u, v)}{\partial v} d u\right\}
$$

где $b$ - гармоническая функция в полуполосе $\{|u|<M, v>0\}$, принимающая при $\{|u|=M, v>2\}$ значение $5 \pi / 4$, а на остальной части границы этой полуполосы ее значения и значения функции $B$ совпадают. Пусть $a=\pi /(2 M)$, а

$$
H(v)=h(v)\left|\frac{(\operatorname{ch} a v+1)\left(\operatorname{ch} a v+\operatorname{ch} a \sigma_{1}\right)\left(\operatorname{ch} a v+\operatorname{ch} a \sigma_{3}\right)\left(\operatorname{ch} a v+\operatorname{ch} a \sigma_{4}\right)}{4 a \operatorname{sh} a \frac{v-\sigma_{3}}{2} \operatorname{sh} a \frac{v+\sigma_{3}}{2} \operatorname{sh} a \frac{v-\sigma_{4}}{2} \operatorname{sh} a \frac{v+\sigma_{4}}{2}\left(\operatorname{ch} a v+\operatorname{ch} a \sigma_{2}\right)}\right|^{1 / 2} .
$$


Тогда

$$
g(v)=f(v) H(v), \quad \text { где } f(v)=\left|\frac{a \operatorname{sh} a \frac{v-\sigma_{2}}{2} \operatorname{sh} a \frac{v+\sigma_{2}}{2}}{\operatorname{sh} a v \operatorname{sh} a \frac{v-\sigma_{1}}{2} \operatorname{sh} a \frac{v+\sigma_{1}}{2}}\right|^{1 / 2} .
$$

Заметим, что $\beta\left(u, \sigma_{4}\right)$, а также $H(v)$ гладко зависят от $\sigma_{1}$ и $\sigma_{2}$, принадлежащих интервалу $(0,1)$. Поэтому, полагая

$$
f_{0}(v)=\left|\frac{v^{2}-\sigma_{2}^{2}}{v\left(v^{2}-\sigma_{1}^{2}\right)}\right|^{1 / 2}, \quad H_{0}=\left.H(v)\right|_{v=\sigma_{1}=\sigma_{2}=0},
$$

получаем, что при $v \leqslant \sigma_{2}$

$$
f(v)=f_{0}(v)\left(1+r_{1}(v)\right), \quad H(v)=H_{0}+r_{2}(v), \quad\left|r_{j}(v)\right| \leqslant K \sigma_{2},
$$

где $r_{j}(v)(j=1,2)$ - гладкая функция, а $K=K(M)$ зависит только от $M$. Заметим, что

$$
L \frac{\sqrt{\sigma_{2}^{2}-\sigma_{1}^{2}}}{\sqrt{\sigma_{1}}}<\int_{0}^{\sigma_{1}} f_{0}(v) d v<L \frac{\sigma_{2}}{\sqrt{\sigma_{1}}}, \quad \text { где } L=\int_{0}^{1} \frac{d v}{\sqrt{v\left|v^{2}-1\right|}} .
$$

Учитывая (1.90), полагаем

$$
\sigma_{1}=\left(\frac{\sigma_{2}}{\alpha}\right)^{2}, \quad \text { где } \alpha \in\left[\alpha_{0}, \frac{1}{\alpha_{0}}\right],
$$

а $\alpha_{0}>\sqrt{\sigma_{2}}$ выберем чуть ниже. Принимая во внимание $(1.81)$ и (1.87)-(1.91), получаем

$$
S_{1}=L H_{0} \alpha+R_{1}, \quad\left|R_{1}\right| \leqslant C_{1} \sigma_{2},
$$

где $C_{1}=C_{1}\left(M, \alpha_{0}\right)$ зависит только от $M$ и $\alpha_{0}$. Кроме того,

$$
\int_{\sigma_{1}}^{\sigma_{2}} f_{0}(v) d v<\alpha\left[L-\int_{\sigma_{\alpha}}^{\infty} \frac{d v}{\sqrt{v\left|v^{2}-1\right|}}\right]<L \alpha-L_{1} \sqrt{\sigma_{2}}
$$

где $\sigma_{\alpha}=\alpha^{2} / \sigma_{2}$, а $L_{1}=L_{1}\left(\alpha_{0}\right)>0$. С другой стороны, для любого $\delta \in(0,1)$

$$
\int_{\sigma_{1}}^{\sigma_{2}} f_{0}(v) d v>\int_{\sigma_{1}}^{\delta \sigma_{2}} f_{0}(v) d v>\alpha \sqrt{1-\delta} \int_{1}^{\delta \sigma_{\alpha}} \frac{d t}{\sqrt{t\left|t^{2}-1\right|}} \rightarrow L \alpha \sqrt{1-\delta}
$$

при $\sigma_{2} \rightarrow 0$. Поэтому

$$
S_{2}=S_{1}-R_{2}, \quad 0<R_{2}<C_{2} \rightarrow 0 \text { при } \sigma_{2} \rightarrow 0,
$$

где $C_{2}=C_{2}\left(M, \alpha_{0}, \sigma_{2}\right)$. Наконец, ввиду (1.81) и (1.85)-(1.86), имеем

$$
S_{3}=\left.\int_{0}^{\sigma_{3}} g(v)\right|_{\sigma_{1}=\sigma_{2}=0} d v+R_{3}, \quad S_{4}=\left.\int_{\sigma_{3}}^{\sigma_{4}} g(v)\right|_{\sigma_{1}=\sigma_{2}=0} d v+R_{4},
$$

где $\left|R_{j}\right|<C_{j}=C_{j}\left(M, \alpha_{0}, \sigma_{2}\right) \rightarrow 0$ при $\sigma_{2} \rightarrow 0$ для $j=3,4$. 
Зафиксируем достаточно малое $\sigma_{2}$. Учитывая (1.91), положим

$$
C\left(\alpha, \sigma_{3}\right) \stackrel{\text { def }}{=} S_{1}+S_{2}-S_{3}-S_{4}, \quad D\left(\alpha, \sigma_{3}\right) \stackrel{\text { def }}{=} S_{1}-S_{2}+S_{3}-S_{4} .
$$

В силу (1.95), для того чтобы соотношения (1.80) были выполнены, достаточно установить разрешимость системы уравнений $C\left(\alpha, \sigma_{3}\right)=D\left(\alpha, \sigma_{3}\right)=0$. С этой целью заметим сначала, что из (1.84) и (1.92)-(1.96) вытекает следующее утверждение.

ПРЕДЛОЖЕНИЕ 1.3. При достаточно малом $\sigma_{2}$ найдется такое $\alpha_{0}<\sqrt{\sigma_{2}}$, что

(i) при любом $\sigma_{3}^{*} \in[1,3 / 2]$ уравнение $C\left(\alpha, \sigma_{3}^{*}\right)=0$ имеет корень $\alpha \in$ $\left[\alpha_{0}, 1 / \alpha_{0}\right]$

(ii) при любом $\alpha^{*} \in\left[\alpha_{0}, 1 / \alpha_{0}\right]$ уравнение $D\left(\alpha^{*}, \sigma_{3}^{*}\right)=0$ имеет корень $\sigma_{3}^{*} \in$ $[1,3 / 2]$.

Кроме того, приращения $\delta_{\alpha} C, \delta_{\sigma_{3}} D$ и $\delta_{\alpha} D, \delta_{\sigma_{3}} D$ функиий $C$ и $D$ относительно аргументов $\alpha$ и $\sigma_{3}$ удовлетворяют следующим соотношениям:

$$
\left|\delta_{\alpha} D\right| \leqslant p\left|\delta_{\alpha} C\right|, \quad\left|\delta_{\sigma_{3}} C\right| \leqslant q\left|\delta_{\sigma_{3}} D\right|,
$$

где $q=q\left(\sigma_{2}\right) \leqslant$ const $u p=p\left(\sigma_{2}\right) \rightarrow 0$ nрu $\sigma_{2} \rightarrow 0$.

Выбрав число $\sigma_{2}$ достаточно малым, будем иметь неравенство $p q<1$. Пусть $\sigma_{3}^{(0)}=1$. Для $k>1$ будем выбирать $\alpha^{(k)}$ и $\sigma_{3}^{(k)}$ так, чтобы $C\left(\alpha^{(k)}, \sigma_{3}^{(k-1)}\right)=0$, $D\left(\alpha^{(k)}, \sigma_{3}^{(k)}\right)=0 . \quad$ Пусть $c_{k} \stackrel{\text { def }}{=} C\left(\alpha^{(k)}, \sigma_{3}^{(k)}\right), d_{k} \stackrel{\text { def }}{=} D\left(\alpha^{(k)}, \sigma_{3}^{(k-1)}\right) . \quad$ В силу $(1.98),\left|c_{k}\right| \leqslant q\left|d_{k}\right|$ и $\left|d_{k+1}\right| \leqslant p\left|c_{k}\right|$. Поэтому $c_{k} \rightarrow 0$ при $k \rightarrow \infty$. Наконец, учитывая очевидную непрерывность функций $C$ и $D$, получаем (1.80).

Для завершения доказательства теоремы 1.6 осталось сослаться на лемму 1.8 , из которой следует однолистность отображения

$$
Q \ni w \mapsto \int_{0}^{w} e^{A+i B} d w \in \Omega^{0} \cap \mathbb{R}_{+},
$$

где $A-$ функция, гармонически-сопряженная к $B$.

1.11. ДоКАЖем теОРему 1.4 о несуществовании $\gamma_{1}$. Легко видеть, что для любой функции $q \in C^{1}[0,1]$ существует такое $M_{0}$, что решение задачи $(1.39)$ в прямоугольнике $Q_{0}=\left\{0<u<M_{0}, 0<v<1\right\}$ при $\chi=\pi / 2$ и $\varphi=N_{+}$ удовлетворяет условию $J(1) \leqslant 0$. Далее, для любого $M<M_{0}$ существует $\sigma_{M}>0$ такое, что если

$$
\varphi(v)= \begin{cases}N_{-} & \text {при } 0<v<\sigma, \\ N_{+} & \text {при } \sigma<v<1\end{cases}
$$

для $\sigma<\sigma_{M}$, то решение задачи (1.39) удовлетворяет условию $J(1)<0$. С другой стороны, предположив существование решения для кривой $\gamma_{1}$, заметим, что функция $B$, определенная в предложении 1.3 , удовлетворяет условию

$$
B(M, v)=N_{-} \quad \text { при } 0<v<\sigma, \text { где } \sigma=\sigma\left(\gamma_{1}\right) \in(0,1) .
$$


Для доказательства теоремы 1.4 остается, ввиду (1.40), показать, что для любого $\sigma_{M}>0$ существует $\varepsilon_{M}>0$ такое, что $\sigma\left(\gamma_{1}\right)<\sigma_{M}$, если $\varepsilon<\varepsilon_{M}$. Для этого заметим, что

$$
\varepsilon \geqslant \int_{0}^{M} e^{A(u, 1)} d u=C_{M} R^{\prime}(1)\left(\int_{0}^{1} H(M, v) d v\right)^{-1},
$$

где

$$
C_{M}=\int_{0}^{M} \exp \left\{\int_{0}^{u} \frac{\partial \beta}{\partial \eta}(\xi, 1) d \xi\right\} d u
$$

а $\beta$-решение задачи (1.39) при $\chi=\pi / 2$ и $N=N_{+}$. Так как $C_{M}>0$, то условие $\varepsilon \rightarrow 0$ влечет $\int_{0}^{1} H(M, v) d v \rightarrow \infty$. Отсюда следует, что $\sigma_{\gamma_{1}} \rightarrow 0$. Теорема 1.4 доказана.

1.12. ДоКАЖем теОРему 1.3 о существовании $\gamma_{2}$. Пусть $\psi=-R^{\prime \prime} / R^{\prime}$, а $B_{\eta, \sigma}$ - решение следующей задачи:

$$
\left.\Delta B_{\eta, \sigma}\right|_{Q}=0,\left.\quad B_{\eta, \sigma}\right|_{v=0}=0,\left.\quad B_{\eta, \sigma}\right|_{u=M}=\varphi,\left.\quad B_{\eta, \sigma}\right|_{v=1}=\chi,\left.\quad \frac{\partial B_{\eta, \sigma}}{\partial u}\right|_{u=0}=\psi,
$$
где

$$
\varphi(v)=\left\{\begin{array}{ll}
N_{+} & \text {при } \sigma<v<1, \\
N_{-} & \text {при } 0<v<\sigma,
\end{array} \quad \chi(u)= \begin{cases}\pi & \text { при } 0<u<\eta, \\
\frac{\pi}{2} & \text { при } \eta<u<M .\end{cases}\right.
$$

Учитывая (1.23), лемму 1.3 , а также то, что $M>M_{0}$, получаем при $v \in(0,1)$ неравенства

$$
0 \stackrel{(1.20)}{=} C_{-}(0, v) \leqslant B_{0,1}(0, v)<B_{\eta, \sigma}(0, v)<B_{M, 0}(0, v) \leqslant C_{0}(0, v) \stackrel{(1.24)}{=} \pi,
$$

из которых вытекает, что $g(0,1)>g(\eta, \sigma)>g(M, 0)$, где

$$
g(\eta, \sigma)=\int_{0}^{1} R^{\prime}(v) \cos B_{\eta, \sigma}(0, v) d v .
$$

Легко видеть, что функция $g:[0, M] \times[0,1] \ni(\eta, \sigma) \mapsto g(\eta, \sigma)$ непрерывна, причем она строго убывает (соответственно возрастает) по первому (соответственно по второму) аргументу.

Заметим далее, что функция $\beta=C_{0}-\pi / 2-B_{0,1}$ является решением задачи

$$
\left.\Delta \beta\right|_{Q}=0,\left.\quad \beta\right|_{v=0}=0,\left.\quad \beta\right|_{u=M}=\varphi^{*},\left.\quad \beta\right|_{v=1}=0,\left.\quad \frac{\partial \beta}{\partial u}\right|_{u=0}=\psi^{*},
$$

где $\varphi^{*}=\max \left(N_{+}-N_{-}-\pi / 2,0\right)$, а $\psi^{*}=\frac{\partial C_{0}}{\partial u}(0, \cdot)$. Поэтому $B_{0,1} \leqslant C_{0}-\pi / 2$ и, значит, $g(0,1)>0$. Но $g(M, 0)<0$. Следовательно, существует единственная кривая в прямоугольнике $\bar{Q}$, вдоль которой функция $g$ обращается в нуль, причем эта кривая задается строго возрастающей функцией

$$
\eta:\left[\sigma_{-}, \sigma_{+}\right] \ni \sigma \mapsto \eta(\sigma) \in\left[\eta_{-}, \eta_{+}\right],
$$

где

$$
0 \leqslant \sigma_{-}<\sigma_{+} \leqslant 1, \quad 0 \leqslant \eta_{-}<\eta_{+} \leqslant M .
$$


Рассмотрим функцию $b=B_{\eta(\sigma), \sigma}$, зависящую от $\sigma \in\left(\sigma_{-}, \sigma_{+}\right)$. Покажем сначала, что функция $b(0, \cdot)-\pi / 2$ обращается в нуль лишь в одной точке. Предположив противное и обозначив через $v_{1}$ наименьший корень уравнения $b(0, v)=\pi / 2$, заметим, что существует такое $v_{*} \in\left(v_{1}, 1\right)$, что $b\left(0, v_{*}\right)=m$, где $m \stackrel{\text { def }}{=} \min _{v_{1} \leqslant v \leqslant 1} b(0, v) \leqslant \pi / 2$. Уравнение $b(0, v)=m$ имеет, очевидно, хотя бы одно решение на $\left[0, v_{1}\right]$. Пусть $v_{0} \leqslant v_{1}$ - максимальный корень этого уравнения. Рассмотрим линию уровня функции $b$, проходящую через $\left(0, v_{0}\right)$, и обозначим через $Q_{0}$ ту часть $Q$, которая лежит выше этой линии. Заметим, что $b \geqslant m$ в $Q_{0}$. Поэтому по лемме о производной гармонической функции в граничной точке минимума получаем $\frac{\partial b}{\partial u}\left(0, v_{*}\right)>0$, т. е. противоречие с $(1.23)$.

Рассмотрим при $\sigma \in\left(\sigma_{-}, \sigma_{+}\right)$функцию $z$, построенную по $B=B_{\eta(\sigma), \sigma}$ согласно формуле (1.49), причем такую, что

$$
z(0)=\int_{0}^{1} e^{A(0, v)} \sin B(0, v) d v+\int_{0}^{\eta} e^{A(u, 1)} d u .
$$

Функция $z$ однолистно отображает $Q$ (ср. лемму 1.8) на односвязную область в первой четверти, имеющую тот же вид, что и область $\Omega$ на рис. $6, \alpha$ ) (см. с. 21). Лишь кривая, соответствующая $\Gamma$, состоит в данном случае из двух отрезков $z(M \times[0, \sigma])$ и $z(M \times[\sigma, 1])$. Продолжив кривую $z(0 \times[0,1])$ симметрично координатным осям, получим, очевидно, искомую свободную границу $\gamma_{2}$.

Положим $\varkappa=\int_{\eta}^{M} \exp \left\{\int_{0}^{u} \frac{\partial B}{\partial v}(\xi, 1) d \xi\right\} d u$ и выясним поведение параметра $\varepsilon_{\sigma}=\varkappa|\gamma|$, где $\sigma \in\left(\sigma_{-}, \sigma_{+}\right)$, при $\sigma \rightarrow \sigma_{-}$и $\sigma \rightarrow \sigma_{+}$. Отметим, что $|\gamma|^{-1}=$ $\int_{0}^{1} H(M, v) d v$, а $\varepsilon_{\sigma}-$ это длина отрезка $[0, z(M+i)]$. Пусть $\sigma \rightarrow \sigma_{-}$. Если $\sigma_{-}=0$, то $|\gamma| \rightarrow 0$, так как $N_{+}>\pi / 2$, a $\varkappa \leqslant$ const. Поэтому $\varepsilon_{\sigma} \rightarrow 0$. Если же $\sigma_{-}>0$, то неинтегрируемых особенностей у функции $H(M, \cdot)$ не возникает. Поэтому длины $\left|\gamma_{-}\right|$и $\left|\gamma_{+}\right|$отрезков $z(M \times[0, \sigma])$ и $z(M \times[\sigma, 1])$, а также величина $\varepsilon_{\sigma}=\left|\gamma_{-}\right| \cos N_{-}+\left|\gamma_{-}\right| \cos N_{+}$остаются отграниченными от нуля. В частности, $\left|\gamma_{+}\right|=1-\left|\gamma_{-}\right| \geqslant$const $>0$. Следовательно, $\lim _{\sigma \rightarrow \sigma_{-}}<\cos N_{-}$.

Пусть теперь $\sigma \rightarrow \sigma_{+}$. Если $\sigma_{+}<1$, то $\eta_{+}=M$. Поэтому $\varkappa$, а следовательно, и $\varepsilon_{\sigma}$ стремится к нулю. Если $\sigma_{+}=1$, то следует различать два подслучая: а) $\eta_{+}=M$ и б) $\eta_{+}<M$. При $\eta_{+}=M$, как и выше, $\varepsilon_{\sigma} \rightarrow 0$. При $\eta_{+}<M$ все зависит от знака $N_{-}$. Если $N_{-}>0$, то особенностей у $H(M, \cdot)$ не возникает. Поэтому $\varepsilon_{\sigma} \rightarrow \cos N_{-}$. Если же $N_{-} \leqslant 0$, то у $H(M, \cdot)$ возникает неинтегрируемая особенность при $\sigma \rightarrow \sigma_{+}$и поэтому $\varepsilon_{\sigma} \rightarrow 0$. Остается отметить тот очевидный факт, что параметр $\varepsilon_{\sigma}$ непрерывно зависит от $\sigma \in\left(\sigma_{-}, \sigma_{+}\right)$. Теорема 1.3 доказана.

ЗАмечАниЕ 1.5. Из доказательства видно, что $\varepsilon_{\sigma} \rightarrow 0$ как при $\sigma \rightarrow \sigma_{-}$, так и при $\sigma \rightarrow \sigma_{+}$, если $\sigma_{-}=0$ и $\eta_{+}=M$. Следовательно, в этом случае существуют два разных контура $\gamma_{2}$ при $\varepsilon<\varepsilon^{*}=\max _{\sigma} \varepsilon_{\sigma}$ (ср. рис. 3 и рис. 5).

Некоторые открытые вопросы. Результат о гладкости [48] кривой $\gamma$ используется в случае $q=1$ в доказательстве единственности [54] кривой $\gamma_{1}$. Но единственность доказана в [54] лишь при условии, что искомая область $\Omega_{1}$ симметрична относительно двух координатных осей. Имеет ли место единственность при $q=1$ в случае, если симметрия предполагается лишь относительно 
одной из осей координат? А если не предполагать какую-либо симметрию контура $\gamma_{1}$, например, в случае, когда внешний контур Г является окружностью? Есть ли единственность кривой $\gamma_{1}$ для произвольного выпуклого контура Г?

\section{§ 2. Явная формула для гармонической функции в терминах ее данных Коши на аналитической кривой и обратная задача о равновесии плазмы}

2.1. Как уже было отмечено в 1 , обратная задача о равновесии плазмы (в ее базовой постановке) - это задача о классификации априори возможных существенно различных типов распределений электрического тока

$$
j: \bar{\omega}=\omega \cup \gamma \ni(x, y) \mapsto j(x, y),
$$

проходящего через искомое сечение $\bar{\omega} \subset \mathfrak{S} \Subset \mathbb{R}^{2}$ плазменного разряда. Заданная область $\mathfrak{S}$ и ее граница $\Gamma=\partial \mathfrak{S}$ имитируют соответственно ортогональные сечения функциональной камеры токамака, приближенно представленной в виде бесконечного цилиндра, и ее металлической оболочки, называемой кожухом. Постановка обратной задачи исходит из следующего. Во-первых, линии уровня так называемой функции магнитного потока $u: \mathbb{R}^{2} \rightarrow \mathbb{R}$, одна из которых есть искомая кривая $\gamma$, совпадают с линиями уровня ее лапласиана. Точнее,

$$
\begin{gathered}
\frac{\partial^{2} u}{\partial x^{2}}+\frac{\partial^{2} u}{\partial y^{2}}= \begin{cases}j(x, y)=f(u(x, y)) \geqslant 0 & \text { при }(x, y) \in \bar{\omega}, \\
0 & \text { при }(x, y) \in \mathfrak{S} \backslash \bar{\omega},\end{cases} \\
\left.u\right|_{\gamma=\partial \omega}=0,\left.\quad u\right|_{\Gamma=\partial \mathfrak{S}}=M,
\end{gathered}
$$

где $M$ - заданное положительное число, а $f$ - искомая функция. Во-вторых,

$$
\int_{\mathfrak{S}} j(x, y) d x d y=1
$$

т. е. известна величина полного тока (принятая за единицу измерения). В-третьих, известны экспериментальные данные $\Psi_{j}$ измерений модуля магнитного поля в ряде точек $P_{j} \in \Gamma$. Это значит, что выполнено условие

$$
\left|\frac{\partial u}{\partial \nu}\right|_{P_{j} \in \Gamma}-\Psi_{j} \mid \leqslant \varepsilon,
$$

где $\varepsilon$ - достаточно малое положительное число, а через $\vec{\nu}$ здесь и ниже обозначается внешняя единичная нормаль к соответствующей замкнутой кривой (в данном случае, к кривой Г).

Целесообразно подразделить обратную задачу на две подзадачи: а) и b), схематично представленные на рис. 10. Подзадача а) относится к "вакуумной" (искомой!) кольцевидной области $\mathfrak{S} \backslash \bar{\omega}$, которую будем здесь обозначать через $\Omega$ (а не через $\Omega^{0}$, как в $\left.\S 1\right)$. В подзадаче а) требуется приближенно найти свободную границу $\gamma=\{u(x, y)=0\}$ искомой области $\omega$ и градиент функции $u$ на $\gamma$. Отметим, что $\left.\nabla u\right|_{\gamma}$ полностью определяется функцией $\psi=\partial u /\left.\partial \nu\right|_{\gamma}$ (ибо $\left.\left.u\right|_{\gamma}=0\right)$. Основные структурные элементы решения подзадачи а) изложены в следующем пункте. 


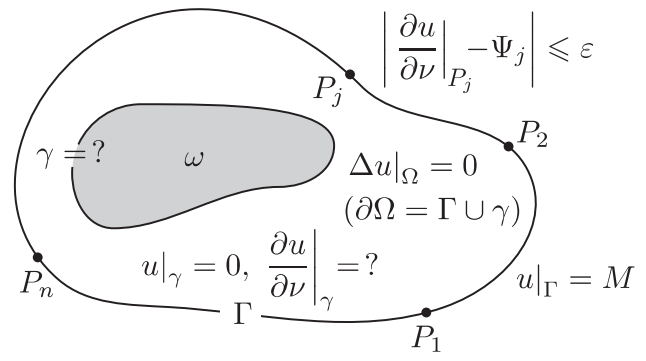

a)

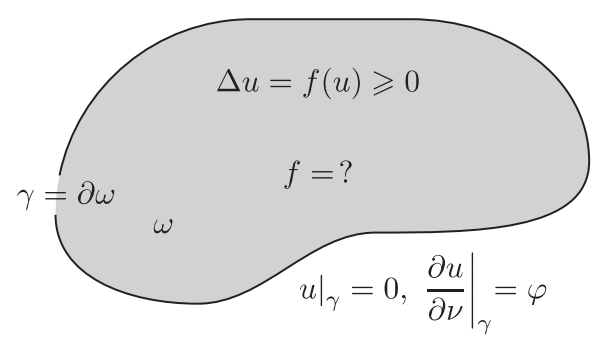

b)

Рис. 10. Две подзадачи обратной задачи о равновесии плазмы (для случая "цилиндрического приближения")

Считая подзадачу а) решенной, можно перейти к подзадаче b). Ее можно сформулировать как проблему поиска в заданной (!) области $\omega$ всех "существенно различных" возможных распределений тока, т. е. отображений

$$
f_{u}: \omega \ni(x, y) \stackrel{(2.1)-(2.2)}{\longmapsto} f(u(x, y)),
$$

удовлетворяющих следующим трем условиям:

$$
\begin{gathered}
\Delta u(x, y) \stackrel{(2.2)}{=} f(u(x, y)) \geqslant 0 \quad \text { в } \omega, \quad u \stackrel{(2.3)}{=} 0 \quad \text { на } \gamma=\partial \omega, \\
\int_{\omega} f(u(x, y)) d x d y \stackrel{(2.4)}{=} 1, \\
\left.\frac{\partial u}{\partial \nu}\right|_{s \in \gamma}=\varphi(s), \quad|\varphi(s)-\psi(s)| \leqslant \kappa .
\end{gathered}
$$

Здесь $\kappa$ - достаточно малое положительное число, а $\psi$ - это та функция на $\gamma$, которая найдена, как и сама кривая $\gamma$, при решении подзадачи а).

Термин, характеризующий “существенное" различие двух отображений $f_{u}^{1}$ и $f_{u}^{2}$, заданных формулой (2.6), четко не определен, поскольку вопрос о важности того или иного различия решается в зависимости от конкретно исследуемой физической характеристики плазменного разряда (ухудшение его удержания, понижение предела устойчивости и пр.). Универсального критерия нет. В каждом случае нужны отдельные и довольно серьезные расчеты, дополненные специальными экспериментами с последующим анализом. С точки зрения физиков, поначалу надо уметь находить распределения, различие профилей которых порядка 10-20-30 процентов “видно на глаз". Это было бы основанием обсуждать проблему дальше и оценивать значение найденных различий в зависимости от конкретной характеристики плазменного разряда. Но в любом случае существенное различие между распределениями $f_{u}^{1}$ и $f_{u}^{2}$ предполагает, что

$$
\frac{\left\|f_{u}^{1}\right\|-\left\|f_{u}^{2}\right\|}{\max \left\{\left\|f_{u}^{1}\right\|,\left\|f_{u}^{2}\right\|\right\}} \geqslant \alpha \sim 0.1 \div 0.3, \quad \text { где }\left\|f_{u}^{j}\right\| \stackrel{\text { def }}{=} \max _{(x, y) \in \omega}\left|f_{u}^{j}(x, y)\right| .
$$

При этом, конечно, с точки зрения физики существенно различными не являются те или иные $\delta$-образные профили, сосредоточенные около некоторого 
подмножества, например, вблизи границы $\gamma$ (что соответствует так называемому скинированному [6] току), хотя такие профили могут иметь существенно различные максимальные значения.

В [6] доказано, что в классе аффинных функций $f: u \mapsto f(u)=a u+b$ при любом $\kappa \stackrel{(2.9)}{>} 0$ имеется бесконечное множество $\left\{f_{u}^{j}\right\}_{j \in \mathbb{N}}$ распределений, которые попарно удовлетворяют неравенству

$$
\left\|f_{u}^{j_{1}}\right\| \ll\left\|f_{u}^{j_{2}}\right\|
$$

а тем самым, и неравенству (2.10) с любым $\alpha<1$. Однако все эти существенно различные в смысле условия (2.11) распределения необходимо [6] являются членами последовательности, сходящейся к $\delta$-функции, сосредоточенной на $\gamma$. Но такие распределения, как уже было отмечено, не являются существенно различными с точки зрения физики. Поэтому в [8] была предпринята попытка численно найти среди полиномов $f: u \mapsto f(u) 3$-й степени отображения $f_{u}^{1}$ и $f_{u}^{2}$, отличные от $\delta$-образных, для которых тем не менее условие (2.11) выполнено. Эта попытка была успешно реализована. Более того, профили построенных отображений $f_{u}^{1}$ и $f_{u}^{2}$ оказались принципиально различными в том абсолютном для физиков смысле, что они удовлетворяют не только условию (2.10), но и условию

$$
(\hat{x}, \hat{y}) \in \operatorname{absmax} f_{u}^{1} \quad \Longrightarrow \quad(\hat{x}, \hat{y}) \in \operatorname{absmin} f_{u}^{2} .
$$

2.2. Опишем теперь основные структурные элементы решения подзадачи а), несколько обобщая ее постановку, а именно рассматривая ее как задачу определения градиента функции $u$ при условии, что

$$
\Delta u=0 \quad \text { в } V_{\Gamma},\left.\quad u\right|_{P_{s} \in \Gamma}=F(s),\left.\quad \frac{\partial u}{\partial \nu}\right|_{P_{s} \in \Gamma}=G(s) .
$$

Здесь $\Gamma$ - аналитическая кривая, для определенности длины $2 \pi$, являющаяся границей односвязной области $\mathfrak{S} \subset \mathbb{R}^{2} \simeq \mathbb{C}, V_{\Gamma}$ - некоторая (двусторонняя) окрестность кривой $\Gamma, \vec{\nu}$ - это внешняя (относительно области $\mathfrak{S}$ ) единичная нормаль в точке $P_{s} \in \Gamma$, а

$$
F: s \mapsto F(s)=\operatorname{Re} \sum_{k \geqslant 0} f_{k} e^{i k s} \quad \text { и } \quad G: s \mapsto G(s)=\operatorname{Re} \sum_{k \geqslant 0} g_{k} e^{i k s}
$$

- это вещественнозначные аналитические функции натурального параметра $s \in \mathbb{R} / 2 \pi$, соответствующего точке $P_{s} \in \Gamma$. Кривая Г полностью определяется заданием $2 \pi$-периодической функции

$$
\mathfrak{N}: \mathbb{T}=\mathbb{R} / 2 \pi \ni s \mapsto \mathfrak{N}(s)=N(s)-s,
$$

где $N(s)$ - угол между осью абсцисс и нормалью $\vec{\nu}$ к $\Gamma$ в точке $P_{s} \in \Gamma$. Как известно, (см., например, [55; § 12]), коэффициенты $L_{k}$ и $M_{k}$ в разложении

$$
\mathfrak{N}(s)=L_{0}+\sum_{k \geqslant 1}\left(L_{k} \cos k s+M_{k} \sin k s\right)
$$

аналитической функции $\mathfrak{N}$ допускают оценку

$$
\left|L_{k}\right| \leqslant C e^{-k \tau}, \quad\left|M_{k}\right| \leqslant C e^{-k \tau},
$$

где константа $C$ мажорирует в $\tau$-окрестности кривой $Г$ модуль аналитической функции $\mathfrak{M}$, ограничением которой на Г является функция $\mathfrak{N}$. 
Ниже выводятся формулы, задающие решение $u$ задачи (2.12) и его градиент в виде степенных рядов с коэффициентами, явно зависящими от параметров $f_{k}$, $g_{k}, L_{k}$ и $M_{k}$, фигурирующих в формулах (2.13)-(2.14). Это позволяет вычислить с любой степенью точности как решение, так и градиент решения этой некорректной (по Адамару) задачи.

Отметим, прежде всего, что формула $V_{\mathbb{T}} \ni \rho e^{i \theta} \mapsto U(\rho, \theta)$, где

$$
U(\rho, \theta) \stackrel{(2.13)}{=} \operatorname{Re}\left(f_{0}+g_{0} \ln \rho+\frac{1}{2} \sum_{k \geqslant 1}\left\{\left(f_{k}+\frac{g_{k}}{k}\right) \rho^{k}+\left(f_{k}-\frac{g_{k}}{k}\right) \rho^{-k}\right\} e^{i k \theta}\right)
$$

задает в некоторой (двусторонней) окрестности $V_{\mathbb{T}} \subset \mathbb{C}$ единичной окружности $\mathbb{T}$ решение такой задачи:

$$
\Delta U=0 \quad \text { в } V_{\mathbb{T}},\left.\quad U(\rho, \theta)\right|_{\rho=1}=F(\theta),\left.\quad \frac{\partial U(\rho, \theta)}{\partial \rho}\right|_{\rho=1}=G(\theta) .
$$

В нижеследующей теореме 2.1 доказывается существование однолистного отображения $\zeta: V_{\Gamma} \ni z \mapsto \zeta(z) \in V_{\mathbb{T}}$ некоторых окрестностей кривой $Г$ и окружности $\mathbb{T}$, для которого

$$
\left|\frac{d \zeta}{d z}\right| \equiv 1 \quad \text { на } Г
$$

Тождество (2.16) означает, что отображение $\zeta$ изометрично на Г. Тем самым, формула

$$
u(x, y)=U(\rho(x, y), \theta(x, y)), \quad \text { где } \rho(x, y) e^{i \theta(x, y)}=\zeta(x+i y),
$$

задает решение задачи (2.12).

TеОРема 2.1 (ср. [56], [57]). В некоторой (двусторонней) окрестности $V_{\mathbb{T}} \subset \mathbb{C}$ единичной окружности $\mathbb{T}$ определено однолистное отображение

$$
z: V_{\mathbb{T}} \ni \zeta=\rho e^{i \theta} \mapsto z(\zeta)=P_{0}+\int_{1}^{\zeta} \exp \{A+i B\} d \zeta \in V_{\Gamma}=z\left(V_{\mathbb{T}}\right), \quad P_{0} \in \Gamma .
$$

Здесъ $A+i B=\ln (d z / d \zeta)$ - аналитическая функиия комплексного переменно-

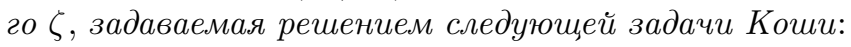

$$
\left.B(\rho, \theta)\right|_{\rho=1}=N(\theta)-\theta,\left.\quad A\right|_{\rho=1}=0 \quad\left(\left.\Rightarrow \frac{\partial B}{\partial \rho}\right|_{\rho=1}=0\right), \quad \rho=|\zeta|, \quad \theta=\arg \zeta
$$

A именно,

$$
\begin{aligned}
& A: V_{\mathbb{T}} \ni \zeta=\rho e^{i \theta} \mapsto A(\rho, \theta)=\frac{1}{2} \sum_{k \geqslant 1}\left(\rho^{k}-\rho^{-k}\right)\left\{M_{k} \cos k \theta-L_{k} \sin k \theta\right\}, \\
& B: V_{\mathbb{T}} \ni \zeta=\rho e^{i \theta} \mapsto B(\rho, \theta)=L_{0}+\frac{1}{2} \sum_{k \geqslant 1}\left(\rho^{k}+\rho^{-k}\right)\left\{L_{k} \cos k \theta+M_{k} \sin k \theta\right\},
\end{aligned}
$$


где $L_{k}$ и $M_{k}$ - коэфбиииенты, фигурирующие в формуле (2.14). При этом отображение (2.18) изометрично на $\mathbb{T}$, m.е.

$$
\left|\frac{d z(\zeta)}{d \zeta}\right| \equiv 1 \quad \text { для } \zeta \in \mathbb{T} .
$$

ДокАзАтЕльство. В силу оценок (2.15), формулы (2.20)-(2.21) задают в некоторой окрестности окружности $\mathbb{T}$ аналитическую функцию $A+i B$, а тем самым, и отображение (2.18). Имеем: $B \stackrel{(2.18)}{=} \arg (d z / d \zeta)$, т. е. $\left.B(\rho, \theta)\right|_{\rho=1}=$ $N(s(\theta))-\theta$, где $s(\theta)$ - длина дуги кривой $\Gamma$, параметризованная точкой $e^{i \theta} \in \mathbb{T}$. Изометрия между $\mathbb{T}$ и Г эквивалентна условию $\left.e^{A}\right|_{\rho=1} \equiv 1$, ибо $\left.e^{A}\right|_{\rho=1}=d s(\theta) / d \theta$. Без ограничения общности можно считать, что $s(\theta)=\theta$. Тем самым, получаем: $N(s(\theta))=N(\theta)$ тогда и только тогда, когда $\left.B(\rho, \theta)\right|_{\rho=1}=N(\theta)-\theta$. Теорема 2.1 доказана.

Теорема 2.2 [58]. Справедливы следующие формулъ:

$$
\begin{aligned}
& \frac{\partial u}{\partial x}(x(\rho, \theta), y(\rho, \theta))=\left(\frac{\partial U(\rho, \theta)}{\partial \rho} \cos N(\rho, \theta)-\frac{1}{\rho} \frac{\partial U(\rho, \theta)}{\partial \theta} \sin N(\rho, \theta)\right) e^{-A(\rho, \theta)}, \\
& \frac{\partial u}{\partial y}(x(\rho, \theta), y(\rho, \theta))=\left(\frac{\partial U(\rho, \theta)}{\partial \rho} \sin N(\rho, \theta)+\frac{1}{\rho} \frac{\partial U(\rho, \theta)}{\partial \theta} \cos N(\rho, \theta)\right) e^{-A(\rho, \theta)} .
\end{aligned}
$$

Здесъ $x(\rho, \theta)+i y(\rho, \theta) \stackrel{(2.18)}{=} z\left(\rho e^{i \theta}\right), A(\rho, \theta)$ u B( $\left.\rho, \theta\right)$ представленьь формулами (2.20) u (2.21), a

$$
\begin{aligned}
& \frac{\partial U}{\partial \rho}=\frac{1}{2} \operatorname{Re} \sum_{k \geqslant 0}\left\{k f_{k}\left(\rho^{k-1}-\rho^{-k-1}\right)+g_{k}\left(\rho^{k-1}+\rho^{-k-1}\right)\right\} e^{i k \theta}, \\
& \frac{\partial U}{\partial \theta}=\frac{1}{2} \operatorname{Im} \sum_{k \geqslant 1}\left\{k f_{k}\left(\rho^{k}+\rho^{-k}\right)+g_{k}\left(\rho^{k}-\rho^{-k}\right)\right\} e^{i k \theta},
\end{aligned}
$$

где $f_{k}$ и $g_{k}$ - коэффичиенть, фигурирующие в формулах (2.13).

ДоказАТЕЛЬство. Имеем: $\zeta=\xi+i \eta, \xi=\rho \cos \theta, \eta=\rho \sin \theta, d z / d \zeta \stackrel{(2.18)}{=}$ $e^{A+i B}$. Поэтому

$$
\frac{\partial x}{\partial \xi}=e^{A} \cos B, \quad \frac{\partial x}{\partial \eta}=-e^{A} \sin B, \quad \frac{\partial y}{\partial \xi}=e^{A} \sin B, \quad \frac{\partial y}{\partial \eta}=e^{A} \cos B .
$$

Следовательно,

$$
\frac{\partial}{\partial \rho}=\cos \theta \frac{\partial}{\partial \xi}+\sin \theta \frac{\partial}{\partial \eta}, \quad \frac{1}{\rho} \frac{\partial}{\partial \theta}=-\sin \theta \frac{\partial}{\partial \xi}+\cos \theta \frac{\partial}{\partial \eta} .
$$

Вспоминая, что $B+\theta \stackrel{(2.19)}{=} N$, получаем

$$
\left(\begin{array}{ll}
\partial x / \partial \rho & \partial x / \partial \theta \\
\partial y / \partial \rho & \partial y / \partial \theta
\end{array}\right) \stackrel{(2.23),(2.24)}{=} e^{A}\left(\begin{array}{cc}
\cos N & -\rho \sin N \\
\sin N & \rho \cos N
\end{array}\right),
$$

в силу чего (вводя обозначения $\left.\rho_{x}=\partial \rho / \partial x, \ldots, \theta_{y}=\partial \theta / \partial y\right)$, имеем

$$
\left(\begin{array}{ll}
\rho_{x} & \rho_{y} \\
\theta_{x} & \theta_{y}
\end{array}\right)=\left(\begin{array}{cc}
\cos N & \sin N \\
-\rho^{-1} \sin N & \rho^{-1} \cos N
\end{array}\right) e^{-A} .
$$

Остается учесть, что $u_{x}=U_{\rho} \rho_{x}+U_{\theta} \theta_{x}$ и $u_{y}=U_{\rho} \rho_{y}+U_{\theta} \theta_{y}$. Теорема 2.2 доказана. 
Выведенные формулы позволяют конструировать линии уровня функции $u$, что требуется в подзадаче а). Отметим еще одно обстоятельство в отношении задачи Коши (2.12). Функцию $G: \Gamma \rightarrow \mathbb{R}_{+}$в (2.12), в контексте подзадачи а), следует рассматривать как интерполяцию экспериментальных измерений $\Psi_{j}$ (см. (2.5)) магнитного поля в $n$ точках $P_{j} \in \Gamma$. При этом на нее нужно наложить ограничения, наследующие свойства функции $\partial u /\left.\partial \nu\right|_{\Gamma}$. А именно, функция $G$ должна иметь равное единице (величине полного тока) среднее значение. Кроме того, $G$ должна быть положительной, ибо в подзадаче а) в качестве функции $F$ из $(2.12)$ взята положительная константа $M$.

Некоторые открытые вопросы. 1. Пусть в формуле (2.9) параметр $\delta$ равен нулю. Тогда существует (см. [5], [7], а также [9]) не более конечного числа распределений $f_{u}$, задаваемых аффинными функциями $f: u \mapsto f(u)=a u+b$, если только односвязная область $\omega$ не круг (для которого, как легко видеть, число распределений континуально). Метод [5] доказательства этого результата базируется на достаточном условии единственности. Им является строгая монотонность функции $\left.a \mapsto \frac{\partial u}{\partial \nu}\right|_{s_{1}}\left(\left.\frac{\partial u}{\partial \nu}\right|_{s_{2}}\right)^{-1}$, где $s_{1}$ и $s_{2}-$ точки на $\gamma$, в которых кривизна кривой $\gamma$ положительна и различна. В [59] доказана единственность решения обратной задачи для некоторого специфического семейства областей $\omega$. Однако пока никому не удалось доказать теорему о том, что для произвольной (или хотя бы для произвольной строго выпуклой) односвязной области $\omega$, отличной от круга, но имеющей гладкую или даже аналитическую границу, может существовать лишь одна пара вещественных чисел $a$ и $b$, для которых разрешима однородная задача Дирихле для уравнения $\Delta u=a u+b \geqslant 0$ при условии, что $\left.\frac{\partial u}{\partial \nu}\right|_{\gamma}=\varphi$, где $\varphi-$ заданная функция.

2. Функцию $G$ в $(2.12)$ естественно выбирать в классе $\mathscr{T}_{n}$ неотрицательных тригонометрических полиномов на Г степени $n-1$ с заданным средним, принимающих в заданных точках $P_{j} \in \Gamma$ заданные значения $\Phi_{j}$ (экспериментальных данных). В [60] приводятся три алгебраических описания множества $\mathscr{T}_{n}$. Одно из этих описаний устанавливает взаимно однозначное соответствие между функциями $G \in \mathscr{T}_{n}$ и точками на некотором эллипсоиде в $\mathbb{C}^{n}$. Интерес в рассматриваемой обратной задаче представляют определяемые выбором функции $G \in \mathscr{T}_{n}$ экстремальные геометрические характеристики искомой свободной границы $\gamma$ (такие, например, как ее кривизна, длина и пр.), а также экстремальные характеристики той или иной нормы градиента $u$ на кривой $\gamma$.

\section{Глава II. Задача Стокса-Лейбензона (для течения Хиле-Шоу)}

\section{§ 3. Теоремы о кратких и бесконечно длительных эволюциях контура}

3.1. Классическая постановка задачи Стокса-Лейбензона такова. Пусть $\Omega_{0}$ - односвязная область в $\mathbb{R}^{2}$, ограниченная достаточно гладкой кривой $\Gamma_{0}$, окружающей начало координат $\{0\}$. Область $\Omega_{0}$ соответствует пятну жидкости в некоторый начальный момент, а источник-сток этой жидкости локализован в начале координат $\{0\} \in \Omega_{0}$. Заданная область деформируется следующим 
образом: в момент $t$ точка $\mathbf{s}(t)=(x(t), y(t))$ границы $\Gamma_{t}$ области $\Omega_{t}$ движется со скоростью $\dot{\mathrm{s}}=(\dot{x}, \dot{y})$, определяемой кинематическим условием

$$
\dot{\mathbf{s}}=\nabla u \quad \text { на } \Gamma_{t},
$$

где $\nabla u=\left(u_{x}, u_{y}\right)$ - градиент функции $u(t ; \cdot, \cdot): \Omega_{t} \rightarrow \mathbb{R}$, удовлетворяющей условию Стокса [11]

$$
u_{x x}+u_{y y}=q \delta(x, y) \quad \text { в } \Omega_{t}
$$

и динамическому условию Лейбензона [12]

$$
u=0 \quad \text { на } \Gamma_{t} .
$$

Здесь $\delta(x, y)$ - это $\delta$-функция Дирака, сосредоточенная в начале координат, а ненулевой коэффициент $q \in \mathbb{R}$ характеризует мощность источника-стока. В дальнейшем мы будем предполагать, что область $\Omega_{t}$ симметрична относительно оси $x$. С учетом этого удобно считать, что $q=2$. При этом $t>0$ будет соответствовать случаю источника, а $t<0$ - случаю стока.

Отметим, вкратце, некоторые результаты исследований задачи (3.1)-(3.3). Они начались с работ Л.А. Галина [61] и П.Я. Полубариновой-Кочиной [62], [63], опубликованных в 1945 г. В этих работах было выведено уравнение

$$
2 \pi \operatorname{Re}\left[\dot{f}(\zeta, t) \overline{\zeta f^{\prime}(\zeta, t)}\right]=q, \quad|\zeta|=1,
$$

для однолистного отображения $f(\cdot, t)$ единичного диска $\{\zeta \in \mathbb{C}|| \zeta \mid \leqslant 1\}$ на искомую область $\Omega_{t}$.

В предположении, что $f(\zeta, t)=a_{1}(t)+a_{2}(t) \zeta+\cdots+a_{n}(t) \zeta^{n}$, в [61] была получена система обыкновенных дифференциальных уравнений на коэффициенты $a_{j}(t)$. В случае $n=2$ эта система принимает вид [63]

$$
a_{1}^{2}(t) a_{2}(t)=a_{1}^{2}(0) a_{2}(0), \quad a_{1}^{2}(t)+2 a_{2}^{2}(t)=a_{1}^{2}(0)+2 a_{2}^{2}(0)-q t \pi^{-1} .
$$

Если $\left|a_{2} / a_{1}\right|<1 / 2$, то образ единичной окружности при отображении $f(\cdot, t)$ есть улитка Паскаля $\Gamma_{0}$ с полярным радиусом $\{r(\theta)=a \cos \theta+b, b>a\}$. В случае стока (т.е. при $q t<0$ ) она за конечное время трансформируется в кардиоиду $\Gamma_{t_{*}}$, острие которой, как оказывается, не достигает точки стока. Далее (т. е. при $q t<q t_{*}$, когда $\left|a_{2} / a_{1}\right|>1 / 2$ ) отображение $f(\cdot, t)$ перестает быть однолистным и решение $t \mapsto \Gamma_{t}$ прекращает свое существование. С помощью мероморфного отображения $\zeta \mapsto f(\zeta, t)=A(t) \zeta /(\zeta-a(t))+b(t) \zeta$ аналогичный эффект установил П.П. Куфарев [64] для окружности, центр которой сдвинут относительно точки стока. Первые теоремы о локальной разрешимости уравнения (3.4) были получены в работе [65].

В работах [61]-[63], [65] задача (3.1)-(3.3) интерпретировалась как течение вязкой жидкости в срезе пористой среды. В последние десятилетия такого рода задачи довольно часто стали называть задачами о течении Хиле-Шоу или просто задачей Хиле-Шоу со свободной границей ${ }^{5}$. Интерес к таким задачам особенно усилился за последние годы (см., в частности, [66]-[71] и цитируемую

\footnotetext{
${ }^{5}$ Парадокс в том, что главные пропагандисты этого термина отмечают в [66] "явную неуместность такого наименования". Печально-противоположная ситуация побудила меня написать дополнение 3 , которым завершается эта статья.
} 
там литературу). Это вызвано не только использованием такого рода течений в инженерной практике, материаловедении, в процессах, связанных с ростом кристаллов (отметим в связи с этим работы [72]-[74]), но и тем, что эти задачи являются хорошими моделями (см., например, [75]-[77]) некоторых весьма сложных двухфазных задач. Поэтому исследование таких моделей позволяет в какой-то мере предугадать особенности главного члена асимптотики решения задач с фазовыми переходами (см., в частности, [78]).

3.2. Важным моментом в исследовании задачи (3.1)-(3.3) было осознание того неожиданного факта, что в случае стока $(q t<0)$ решение этой задачи не существует ни при каком сколько угодно малом $t$, если начальный контур $\Gamma_{0}$ не аналитичен хотя бы в одной точке. Другими словами, при таком начальном контуре сток невозможен: задача не имеет решения. Причина этого разъяснена ниже. А может ли такой (неаналитический) контур сдвинуться в случае источника? Ответ на этот вопрос был получен сравнительно недавно в [79], [80], где было доказано, что при малых временах решение задачи (3.1)-(3.3) в случае источника существует. На первый взгляд, все это удивительно, ибо закон движения контура $\Gamma_{0}$ априори никак не зависит ни от его аналитичности, ни от типа "двигателя": источник или сток. Внутренняя причина этих фактов ниже выявлена при рассмотрении эволюции гармоник слабого возмущения окружности.

Введем в $\Omega_{t}^{+}=\Omega_{t} \cap \mathbb{R}_{+}^{2}$ функцию $v$, гармонически-сопряженную функции $u$. Запараметризуем точку $\mathbf{s}(t) \in \Gamma_{t}^{+}=\Gamma_{t} \cap \mathbb{R}_{+}^{2}$ числом $\eta$, задающим ту линию уровня $\left\{(x, y) \in \Omega_{t} \mid v(t ; x, y)=\eta\right\}$ функции $v$, которая содержит эту точку $\mathbf{s}(t)$. Ввиду симметрии $\Omega_{t}$ и выбранного (по этой причине) значения $q=2$, параметр $\eta$ меняется от нуля до единицы. Тем самым, определена непрерывная функция

$$
s(t, \cdot):[0,1] \ni \eta \mapsto s(t, \eta)=\left|P_{0} P_{\eta}\right| \in\left[0,\left|\Gamma_{t}^{+}\right|\right],
$$

где $\left|P_{0} P_{\eta}\right|$ - длина дуги $\widetilde{P_{0} P_{\eta}}$ кривой $\Gamma_{t}^{+}$, отсчитываемая в положительном направлении от точки $P_{0}$ пересечения кривой $\Gamma_{t}^{+}$с положительной полуосью $x$ до точки $P_{\eta}$ пересечения этой кривой с линией уровня $\{v(t ; x, y)=\eta\}$.

Определим (при каждом $t$ ) в замыкании полуполосы

$$
Q=\{u+i v \in \mathbb{C} \mid-\infty<u<0,0<v<1\}
$$

функцию Гельмгольца-Кирхгофа формулой

$$
A+i B: w=u+i v \mapsto A(t ; u, v)+i B(t ; u, v)=\ln \left|\frac{\partial z(t ; w)}{\partial w}\right|+i \arg \frac{\partial z(t ; w)}{\partial w},
$$

где $z=x+i y \in \Omega_{t}^{+}$. Имеем:

$$
x(t, v)=x(t, 0)-\int_{0}^{v} e^{a(t, \eta)} \sin b(t, \eta) d \eta, \quad y(t, v)=\int_{0}^{v} e^{a(t, \eta)} \cos b(t, \eta) d \eta,
$$

где $x(t, 0)=\int_{-\infty}^{0} e^{A(t ; u, 0)} d u, \mathrm{a}$

$$
a(t, v)+i b(t, v)=A(t ; 0, v)+i B(t, 0, v) .
$$


Таким образом, задача (3.1)-(3.3) может быть переформулирована в терминах функций $a$ и $b$. А именно [81], задача (3.1)-(3.3) эквивалентна задаче Коши для нелинейного интегро-дифференциального уравнения

$$
\dot{b}(t, v)=e^{-2 a} a^{\prime}+b^{\prime} e^{-a} \int_{0}^{v}\left[e^{a} \dot{a}-e^{-a} b^{\prime}\right] d \xi, \quad \dot{b} \stackrel{\text { def }}{=} \frac{\partial b}{\partial t}, \quad b^{\prime} \stackrel{\text { def }}{=} \frac{\partial b}{\partial v},
$$

относительно функций $a$ и $b$, связанных между собой (при каждом $t$ ) преобразованием Гильберта: согласно (3.8), функции $a$ и $b$ являются следом при $u=0$ гармонически-сопряженных в полуполосе $Q$ функций $A$ и $B$. По существу, именно это обстоятельство является препятствием для существования решения в случае стока при отсутствии аналитичности кривой $\Gamma_{0}$ хотя бы в одной точке. Дело в том, что уравнение (3.9) жестко связывает возможность эволюции функции $t \rightarrow b(t, v)$, т. е. эволюции угла наклона касательной к $\Gamma_{t}$ в точке $P_{v} \in \Gamma_{t}$, с эволюцией коэффициента продольной деформации $e^{a(t, v)}$ контура в этой точке. В случае стока (т. е. при $t<0$ ), когда контур “съеживается", продольная деформация контура в точке $P_{v} \in \Gamma_{0}$, определяемая уравнением (3.9), оказывается невозможной, если в этой точке контур $\Gamma_{0}$ не аналитичен. Уравнение (3.9) в этом случае (при любом сколь угодно малом $-t>0$ ) неразрешимо по причине, вскрытой в теореме 3.2 и связанной с экспоненциальным убыванием коэффициентов Фурье аналитической функции на окружности.

3.3. Заметим, что $B(t, u, v) \equiv \pi v$, если (и только если) кривая $Г$ является окружностью $\left\{z \in \mathbb{C}|| z \mid=R_{0}>0\right\}$, центр которой совпадает с началом координат (т. е. носителем $\delta$-функции в уравнении (3.2)). Учитывая это, представим функцию $b$ в виде

$$
b(t, v)=\pi v+\beta(t, v) .
$$

Заметим, что $\beta(t, 0)=\beta(t, 1)=0$ (в силу симметрии и дифференцируемости кривой $\Gamma)$, и разложим функцию $\beta(t, \cdot):[0,1] \ni v \mapsto \beta(t, v)$ в ряд Фурье

$$
\beta(t, v)=\sum_{k \geqslant 1} \beta_{k}(t) \sin \pi k v
$$

по базису $e_{k}:[0,1] \ni v \mapsto \sin \pi k v, k \in \mathbb{N}$, пространства $L^{2}(0,1)$.

Легко видеть, что те же коэффициенты Фурье $\beta_{k}(t)$ определяют и функцию $a_{0}(t)$, задаваемую равенством

$$
A(t ; u, v)=a_{0}(t)+\pi u+\sum_{k \geqslant 1} \beta_{k}(t) e^{\pi k u} \cos \pi k v
$$

что, очевидно, можно выразить и таким равенством:

$$
a(t, v)=a_{0}(t)+\alpha(t, v), \quad \text { где } \alpha(t, v)=\sum_{k \geqslant 1} \beta_{k}(t) \cos \pi k v .
$$

Действительно, приращение площади $\left|\Omega_{t}\right|=2\left|\Omega_{t}^{+}\right|$области $\Omega_{t}$ за единицу времени равно

$$
\int_{\Gamma} \dot{\mathbf{s}} d \Gamma \stackrel{(3.1)}{=} \int_{\Gamma} \frac{\partial u}{\partial \nu} d \Gamma
$$


т. е. коэффициенту при $\delta$-функции в уравнении (3.2). Другими словами,

$$
\frac{d}{d t}\left|\Omega_{t}^{+}\right|=1 \quad \Longleftrightarrow \quad\left|\Omega_{t}^{+}\right|=t+t_{0}, \quad \text { где } t_{0}=\left|\Omega_{0}^{+}\right| .
$$

В терминах функции Гельмгольца-Кирхгофа это условие принимает вид

$$
\frac{\partial}{\partial t} \int_{0}^{1}\left(\int_{-\infty}^{0} e^{2 A(t ; u, v)} d u\right) d v=1
$$

поскольку якобиан отображения $w \mapsto e^{A+i B}$ равен $|\partial z / \partial w| \stackrel{(3.6)}{=} e^{2 A}$. Отсюда следует, что коэффициент $e^{a(t, v)}$ продольной деформации контура Г определяется согласно (3.14). Поэтому он, а значит, и функция $a_{0}(t)$ зависят от $\left|\Omega_{0}\right|$ и коэффициентов Фурье $\beta_{k}(t)$.

Таким образом, искомая деформация начальной кривой $\Gamma_{0}$, определяемая задачей Коши для уравнения (3.9), полностью характеризуется эволюцией коэффициентов Фурье $\beta_{k}(\cdot)$ в разложении $\beta(t, v)=\sum_{k \geqslant 1} \beta_{k}(t) \sin \pi k v$.

Теорема 3.1 [82]. Уравнение (3.9) представимо в виде

$$
\dot{\beta}-\mathbf{K}(\beta) \dot{\beta}=\frac{1}{2\left(t+t_{0}\right)} \mathbf{F}(\beta) .
$$

Здесъ $\mathbf{K}=\mathbf{K}_{0}+\mathbf{K}_{1}, \mathbf{F}=\mathbf{F}_{0}+\mathbf{F}_{1}$, где

$$
\begin{aligned}
{\left[\mathbf{K}_{0}(\beta) \dot{\beta}\right](t, v) } & =b^{\prime}(t, v) e^{-\alpha(t, v)} \int_{0}^{v} e^{\alpha(t, \eta)} \dot{\alpha}(t, \eta) d \eta \\
{\left[\mathbf{K}_{1}(\beta) \dot{\beta}\right](t, v) } & =\left(\sum_{j \geqslant 1} \frac{2 \beta_{j} \dot{\beta}_{j}}{j+1}\right) \sum_{k \geqslant 1} \frac{(-1)^{k-1}}{k} \sin \pi k v+\left[\mathbf{r}_{1}(\beta) \dot{\beta}\right](t, v), \\
{\left[\mathbf{F}_{0}(\beta)\right](t, v) } & =\frac{1}{\pi}\left\{e^{-2 \alpha} \alpha^{\prime}-b^{\prime} e^{-\alpha} \int_{0}^{v} e^{-\alpha} b^{\prime} d \eta+\pi b^{\prime} e^{-\alpha} \int_{0}^{v} e^{\alpha} d \eta\right\} \\
{\left[\mathbf{F}_{1}(\beta)\right](t, v) } & =\left(\sum_{j \geqslant 1} \frac{2 \beta_{j}^{2}}{j+1}\right) \sum_{k \geqslant 1} \frac{(-1)^{k-1}}{k} \sin \pi k v+\left[\mathbf{s}_{1}(\beta)\right](t, v) .
\end{aligned}
$$

При этом

$$
\left|\mathbf{r}_{1}(\beta) \dot{\beta}\right| \leqslant C\|\beta\|_{1}^{2}\|\beta\|_{0}, \quad\left|\mathbf{s}_{1}(\beta)\right| \leqslant C\|\beta\|_{1}^{3},
$$

¿əe

$$
\|\beta\|_{1}=\max _{t} \sqrt{\sum_{k \geqslant 1}\left(k \beta_{k}(t)\right)^{2}}, \quad\|\dot{\beta}\|_{0}=\max _{t} \sqrt{\sum_{k \geqslant 1}\left(\dot{\beta}_{k}(t)\right)^{2}} .
$$

Покоординатно динамическая система (3.16) въцлядит так:

$$
\left.\begin{array}{c}
2\left(t+t_{0}\right)\left(\beta_{1} \dot{\beta}_{1}+r_{1}(\beta) \dot{\beta}\right)=\left(-\beta_{1}^{2}+2 \sum_{j \geqslant 2} \beta_{j}^{2}\right)+s_{1}(\beta), \\
\left.t+t_{0}\right)\left(\dot{\beta}_{k}+r_{k}(\beta) \dot{\beta}\right)=-(k+2) \beta_{k}+s_{k}(\beta) \quad \partial л я k \geqslant 2,
\end{array}\right\}
$$

где

$$
\left|r_{k}(\beta) \dot{\beta}\right| \leqslant C\|\beta\|_{1}^{1+\operatorname{sign}|k-1|}\|\dot{\beta}\|_{0}, \quad\left|s_{k}(\beta)\right| \leqslant C\|\beta\|_{1}^{2+\operatorname{sign}|k-1|}
$$


Из теоремы 3.1 с помощью кропотливых функционально-геометрических построений может быть выведено следующее утверждение.

ТеОрема 3.2 [81]-[83]. Если начальный контур $\Gamma_{0}$ есть малое (определяемое формулами (3.20)) возмущение окружности радиуса $R(0)$, то в случае источника его эволюиия $\Gamma_{t}$ продолжается бесконечно долго и она единственна на любом временном интервале. Что же касается отклонения $\Gamma_{t}$ от окружности радиуса $R(t)=\sqrt{R^{2}(0)+2 t / \pi}$, то, начиная с некоторого момента, оно почти монотонно стремится $\kappa$ нулю.

Точнее, существует число $\rho \in(0,1 / 8)$ такое, что для любого $\mu \in(0,1)$ функиия

$$
[0,1] \ni v \mapsto b(t, v)=\pi v+\beta(t, v), \quad \text { əде } \beta(t, v)=\sum_{n \geqslant 1} \beta_{n}(t) \sin \pi n v,
$$

определяющая контур $\Gamma_{t}$, существует при любом $t>0$, если только начальная угловая функиия, задающая контур $\Gamma_{0}$, m.е. функиия

$$
[0,1] \ni v \mapsto b(0, v)=\pi v+\sum_{n \geqslant 1} \beta_{n}^{0} \sin \pi n v,
$$

удовлетворяет условию

$$
\sum_{k \geqslant 2}\left(k \beta_{k}^{0}\right)^{2} \leqslant(\mu \rho)^{1 / 2}\left|\beta_{1}^{0}\right|^{3 / 2}, \quad 0<\left|\beta_{1}^{0}\right| \leqslant \mu \rho .
$$

При этом

$$
\left|\dot{\beta}_{1}(0)-\dot{\bar{\beta}}_{1}(0)\right| \leqslant \mu, \quad \sqrt{\sum_{k \geqslant 2}\left|\dot{\beta}_{k}(0)-\dot{\bar{\beta}}_{k}(0)\right|^{2}} \leqslant \mu,
$$

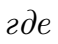

$$
\begin{aligned}
& \bar{\beta}_{1}^{2}(t)=\frac{1}{t+t_{0}}\left(t_{0} \beta_{1}^{2}(0)+2 \sum_{k \geqslant 2} \beta_{k}^{2}(0) \int_{0}^{t} \frac{d \tau}{\left(1+\tau / t_{0}\right)^{k+2}}\right), \\
& \bar{\beta}_{k}(t)=\frac{\beta_{k}(0)}{\left(1+t / t_{0}\right)^{k / 2+1}} .
\end{aligned}
$$

Кроме того, существует константа С (немного превосходящая единищу) такая, что при любом $t \geqslant 0$ справедливы оченки

$$
\left|\dot{\beta}_{1}(t)-\dot{\bar{\beta}}_{1}(t)\right| \leqslant C \mu, \quad \sqrt{\sum_{k \geqslant 2}\left|\dot{\beta}_{k}(t)-\dot{\bar{\beta}}_{k}(t)\right|^{2}} \leqslant C \mu .
$$

ЗАмЕчаниЕ 3.1. Формулы (3.23) объясняют природу отмеченного выше результата: если в случае $q t<0$, т. е. стока (!), задача (3.1)-(3.3) разрешима хотя бы при сколь угодно малом $t$ (в нашем случае $q=2>0$ это означает, что речь идет о любом малом отрицательном $t$ ), то начальный контур $\Gamma_{0}$ необходимо аналитичен. Действительно, в силу (3.23) функция (3.18) будет определена при сколь угодно малом $t<0$ лишь тогда, когда коэффициенты Фурье $\beta_{k}^{0}=\beta_{k}(0)$ функции (3.19) экспоненциально быстро убывают и определяют тем самым (см., например, [55; §12]) аналитическую функцию. 
Отметим также, что последнее утверждение (о бесконечном по времени "дрейфе" возмущенной окружности; ср. с [84]) согласуется с таким фактом: если при деформации $t \mapsto \Gamma_{t}$ кривая $\Gamma_{t_{*}}$ при некотором конечном $t_{*} \neq 0$ гомотетична окружности с центром в начале координат $\{0\}$, т.е. там, где локализован источник-сток, то кривая $\Gamma_{0}$, а потому и все кривые $\Gamma_{t}$ гомотетичны той же самой окружности. Иными словами, справедлива следующая теорема.

ТЕОРема 3.3. Если $\Gamma_{0}$ стягивается $\kappa$ стоку, то $\Gamma_{0}$ есть окружность $c$ центром в точке стока.

ДокАЗАТЕЛЬСтво. Согласно [85], при $n \geqslant 1$ все комплексные моменты

$$
M_{n} \stackrel{\text { def }}{=} \int_{\Omega_{t}} z^{n} \overline{d z} \wedge d z
$$

не зависят от $t$. Следовательно, они равны нулю, поскольку $\left|\Omega_{t}\right| \rightarrow 0$ в процессе стягивания контура $\Gamma_{0}$ в точку стока. Далее, $M_{0}=2 i\left|\Omega_{t}\right|$. Кроме того, $M_{n}=\int_{\Gamma_{t}} z^{n} \bar{z} d z=2 \pi i c_{-(n+1)}$, где $c_{k}-$ коэффициенты в разложении в ряд Лора-

на $\sum c_{k} z^{k}$ функции $f$, аналитической в $\Omega_{t} \backslash\{z=0\}$ и принимающей по непрерывности на $\Gamma_{t}$ значения функции $\bar{z}$. Следовательно, $f(z)=c_{-1} / z+g(z)$, где $g$ - аналитическая функция в $\Omega_{t}$. Тем самым, имеем следующую цепочку импликаций на $\Gamma_{t}$ :

$$
\left(|z|^{2}-c_{-1}=z g(z)\right) \Rightarrow\left(\operatorname{Im}(z g(z))=\frac{\left|\Omega_{t}\right|}{\pi}\right) \Rightarrow\left(z g(z)=(a+i b)\left|\Omega_{t}\right|\right) .
$$

А поскольку $\left.z g(z)\right|_{z=0 \in \Omega}=0$, то $a+i b=0 \Rightarrow g(z) \equiv 0$. Поэтому $|z|^{2}=\left|\Omega_{t}\right| / \pi$ на $\Gamma_{t}$, а это значит, что $\Omega_{t}$ есть диск с центром в нуле.

СлеДСтвиЕ 3.1. Если при деформации $t \mapsto \Gamma_{t}$ две кривые $\Gamma_{0} u \Gamma_{t_{*}} c t_{*} \neq 0$ оказались гомотетичными, то $\Gamma_{0}$ гомотетична окружности с иентром 8 точке источника-стока.

Действительно, $\Gamma_{t_{*}}$, а потому и $\Gamma_{0}$ стягиваются в точку, ибо, предположив для определенности, что $\Gamma_{t_{*}}$ находится внутри $\Gamma_{0}$, получаем, что кривая $\Gamma_{n t_{*}}$ попадает в сколь угодно малую окрестность начала координат при достаточно большом $n \in \mathbb{N}$.

\section{§ 4. “Пальцевая" неустойчивость Кочиной-Саффмана-Тейлора}

Теорема 3.2 проясняет эволюцию слабых возмущений окружности. В случае произвольного начального контура уравнение (3.16) изучалось в рамках аппроксимационной так называемой квазиконтурной [86]-[88] (ранее именовавшейся полигональной [89], конечноточечной [90]) модели задачи (3.1)-(3.3). Квазиконтурная модель описывается матричным уравнением вида

$$
Q(N, \sigma) \dot{N}=P(t, N, \sigma),
$$

аппроксимирующим нелинейное интегро-дифференциальное уравнение (3.16). Здесь $N=\left(N_{1}, \ldots, N_{m}\right), N_{j}$ - угол между осью $x$ и внешней нормалью к $j$-й стороне полигонального квазиконтура, а параметр $\sigma=\left(\sigma_{1}, \ldots, \sigma_{m}\right)$ характери- 
зует длины его сторон. Численный анализ [86]-[88] показал, что в пространстве квазиконтуров имеется гиперповерхность

$$
\mathfrak{N}_{m}=\{(N, \sigma) \mid \operatorname{det} Q(N, \sigma)=0\} .
$$

Эта поверхность коразмерности 1 является в случае источника $(t>0)$ притягивающим многообразием (аттрактором). При подходе к $\mathfrak{N}_{m}$ скорость движения экспоненциально возрастает. Дальнейшее движение может интерпретироваться как сверхбыстрые осцилляции около $\mathfrak{N}_{m}$ с бесконечно малой амплитудой и весьма медленным продвижением в направлении одного из притягивающих центров на самом аттракторе. Один из этих центров - квазиокружность, т. е. правильный $m$-угольник $(\widehat{N}, \hat{\sigma})$. Если обратить время, т. е. рассмотреть случай стока, то эта гиперповерхность превращается в отталкивающее многообразие, как говорят, репеллер (антиаттрактор). Было бы правильнее сказать, в суперрепеллер, поскольку вполне “приличный", внешне ничем особо не выделяющийся контур $\Gamma_{0}$, представленный точкой $P_{0}$, расположенной очень близко к этой гиперповерхности, почти мгновенно изменяет свою форму. Дело в том, что точка $P_{0}$ с громадной скоростью "отлетает" от гиперповерхности и через мгновение находится вдали от нее, представляя контур, существенно отличный от $\Gamma_{0}$. Отличие обусловлено тем, что сильно растут гармоники, соответствующие вырождению оператора $\mathbf{I}-\mathbf{K}$, стоящему при $\dot{\beta}$ в уравнении (3.16). При этом площадь, охватываемая контуром, за столь короткое время почти не уменьшается (так как мощность стока постоянна). Поэтому возникают “пальцы" [14], [15], компенсирующие скачки́ кривизны контура (вызванные ростом некоторых гармоник) при почти постоянной площади, ограниченной этим контуром.

Некоторые открытые вопросы. 1. Теорема 3.2 не охватывает все близлежащие к окружности начальные положения контура $\Gamma_{0}$, точнее: данные Коши для возмущения функции $\beta$ не принадлежат полной окрестности нуля пространства $H^{1}(0,1)$. Исключен случай, когда

$$
\left|\beta_{1}^{0}\right|^{3 / 2}=o\left(\beta^{*}\right) \quad \text { при } \beta^{*} \stackrel{\text { def }}{=} \sum_{k \geqslant 2}\left(k \beta_{k}^{0}\right)^{2} \rightarrow 0 .
$$

Как устроено векторное поле траекторий $\left(\dot{\bar{\beta}}_{1}, \dot{\bar{\beta}}_{k}\right)$ в области, определяемой условием (4.1)?

2. Аттрактирующая (с ростом $t$ ) поверхность $\mathfrak{N}_{\infty}$ характеризуется тем, что на ней вырождается оператор $\mathbf{I}-\mathbf{K}$, стоящий при $\dot{\beta}$ в уравнении (3.16). Как в терминах оператора $\mathbf{I}-\mathbf{K}$ охарактеризовать притягивающие центры на $\mathfrak{N}_{\infty}$ ?

\section{Глава III. Задачи со свободной границей на экстремум}

\section{§5. Плоские течения с минимальным отношением экстремальных значений давления на свободной границе}

5.1. В качестве одного из примеров задачи о плоском стационарном потенциальном течении несжимаемой жидкости (или, в другой интерпретации, о стационарном тепловом потоке) с минимальным отношением экстремальных 
значений модуля скорости на свободной границе $\gamma$ в этом параграфе излагается та часть работы [91], которая посвящена задаче, возникшей в АНTK (авиационном научно-техническом комплексе) им. А.Н. Туполева. Эта задача была связана с проблемой обледенения элементов корпуса летательных аппаратов.

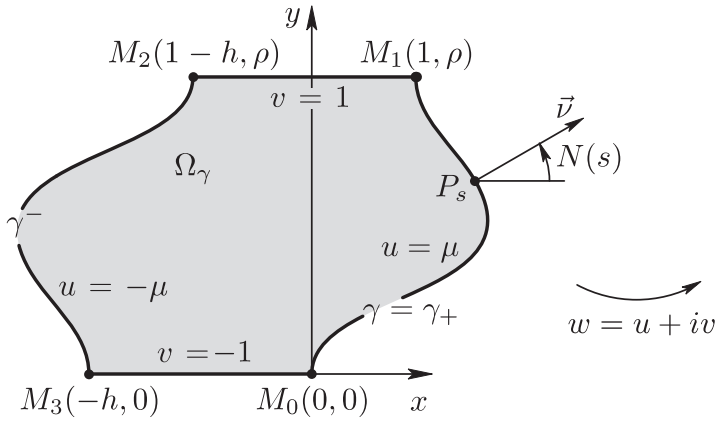

a)

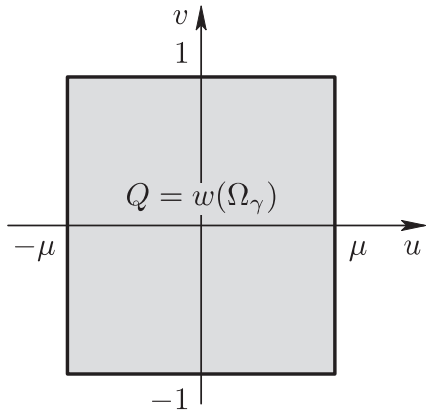

b)

Рис. 11. а) Область $\Omega=\Omega_{\gamma} ; N(s)$ - угол между осью $x$ и нормалью $\vec{\nu}$ к $\Gamma$ в точке $P_{s} \in \gamma$. b) Прямоугольник $Q=w(\Omega)$ - это образ области $\Omega$ при однолистном отображении $w=u+i v$, подчиненном условию (5.2).

Суть дела сводилась к поиску такой (см. рис. 11) односвязной центрально-симметричной области $\Omega_{\gamma}$, ограниченной отрезками $M_{3} M_{0}, M_{1} M_{2}$ и кривыми $\gamma=\gamma_{+}$и $\gamma_{-}$, для которой минимальна следующая величина:

$$
\Phi(\gamma)=\max _{P \in \gamma}\left|\frac{d w}{d z}(P)\right|\left(\min _{P \in \gamma}\left|\frac{d w}{d z}(P)\right|\right)^{-1},
$$

где $w=u+i v$ - такое однолистное отображение области $\Omega=\Omega_{\gamma} \subset \mathbb{C}$ на прямоугольник $Q=\{|u|<\mu,|v|<1\}$, которое подчинено условию

$$
u= \pm \mu \text { на } \gamma_{ \pm}, \quad v=-1 \text { на } M_{3} M_{0} \quad \text { и } v=1 \text { на } M_{1} M_{2},
$$

где кривая $\gamma=\gamma_{+}$соединяет точки $M_{0}=(0,0), M_{1}=(1, \rho)$, а центрально-симметричная ей кривая $\gamma_{-}-$точки $M_{2}=(1-h, \rho)$ и $M_{3}=(-h, 0)$. Здесь $h=h_{\gamma}-$ положительное число, зависящее от заданного параметра $\mu$ и кривой $\gamma$. Отметим, что такое $h=h_{\gamma}$ существует, если при уменьшении $h$ (до тех пор пока $\gamma$ и $\gamma$ - не соприкоснутся) интеграл $I(h, \gamma)=\int_{\gamma} \frac{\partial u}{\partial \nu} d \gamma$, равный $v\left(M_{1}\right)-v\left(M_{0}\right)$, превысит значение 2. Действительно, интеграл $I(h, \gamma)$ непрерывно зависит от $h$ и стремится к нулю при $h \rightarrow \infty$. Отметим также, что если $h$ можно уменьшать до нуля, то $\sup _{(h, \gamma)} I(h, \gamma)=\infty$.

Если зеркально отразить область $\Omega_{\gamma}$ относительно отрезков $M_{3} M_{0}, M_{1} M_{2}$ и последовательно так отражать возникающие области, то получится криволинейная периодическая полоска "ширины” $h$. Ее полупериод равен $\rho$. По такой полоске, вырезанной из фольги, можно пропустить электрический ток $J=\nabla v$. При прохождении тока по полоске фольги в ней возникает тепловой поток. В точке $P_{0} \in \Omega$ величина этого теплового потока пропорциональна $\left|\nabla u\left(P_{0}\right)\right|$. 
Рассмотрим в $\Omega$ криволинейную систему координат $(\vec{\nu}, \vec{\tau})$, порожденную градиентами функций $u$ и $v$. Функция $\partial u / \partial \nu$ гармонична в $\Omega_{\gamma}$ и непрерывна в ее замыкании. Поэтому экстремальные значения теплового потока достигаются на границе полоски. Таким образом, чтобы максимально сократить риск перегорания полоски фольги, нужно так подобрать кривую $\gamma$, чтобы на (порожденной ею) границе полоски фольги, которую мы обозначим через Г, была минимальна следующая величина: $\max _{P \in \Gamma}|\nabla u(P)| / \min _{P \in \Gamma}|\nabla u(P)|$.

Другая интерпретация задачи о минимизации функционала (5.1) может быть связана с поиском такого (вызванного технологическими причинами) искривления профиля трубопровода, при котором на его стенках минимально отношение экстремальных значений давления установившегося течения идеальной жидкости. Еще одна интерпретация такой задачи заключается в выборе такой формы плоской пористой (например, фильтрующей или катализирующей) структуры $\Omega$, для которой выполнено следующее условие: пропускаемый через область $\Omega$ потенциальный бездивергентный поток минимально отклоняется от равномерного распределения на "выходе" из области $\Omega$. При этом "выходом" является искомая свободная граница $\gamma=\partial \Omega \backslash \Gamma$ (или, быть может, ее часть).

5.2. В этом пункте мы сначала уточним класс рассматриваемых кривых $\gamma$ и получим формулу для кривой $\hat{\gamma}$, доставляющей минимум функционалу (5.1), предполагая существование кривой $\hat{\gamma}$. Затем будет представлена теорема о существовании этой кривой.

В разделе 37 учебника [46] приведен простой пример кусочно аналитической кривой $\Gamma$ класса $C^{1}$ (в точке сопряжения аналитических дуг), для которой $\Phi(\Gamma)=\infty$. Ограничимся поэтому кривыми $\Gamma$ класса $C^{k+1, \lambda}$, фиксируя то или иное целое $k \geqslant 0$ и число $\lambda \in(0,1)$. Это эквивалентно тому, что функция $N: \gamma \ni$ $P_{s} \mapsto N(s)$, задающая угол $N(s)$ (см. рис. 11, а)) между осью $x$ и нормалью к кривой $\gamma$ в точке $P_{s} \in \gamma$, отстоящей вдоль $\gamma$ на расстоянии $s$ от точки $M_{0}$, удовлетворяет двум условиям:

1) $N(0)=N(|\gamma|)=0$ (т. е. кривая $\Gamma$ пересекается под прямым углом с отрезками $M_{3} M_{0}$ и $\left.M_{1} M_{2}\right)$;

2) $N \in C^{k, \lambda}(I)$, где $I=[0,|\gamma|]$, а $|\gamma|$ - длина кривой $\gamma$.

Напомним, что норма в $C^{k, \lambda}(I)$ есть $\|f\|_{k, \lambda} \stackrel{\text { def }}{=}\|f\|_{k}+\left[f^{(k)}\right]_{\lambda}$, где

$$
\|f\|_{k}=\max _{0 \leqslant j \leqslant k} \sup _{t \in I}\left|f^{(j)}(t)\right|, \quad\left[f^{(k)}\right]_{\lambda}=\sup _{t_{i} \in I, 0<\left|t_{1}-t_{2}\right| \leqslant 1} \frac{\left|f^{(k)}\left(t_{1}\right)-f^{(k)}\left(t_{2}\right)\right|}{\left|t_{1}-t_{2}\right|^{\lambda}},
$$

a $f^{(j)}-j$-я производная функции $f$.

Зафиксировав целое $k \geqslant 0$ и $\lambda \in(0,1)$, будем рассматривать задачу минимизации функционала (5.1) на множестве $\mathscr{G}=\mathscr{G}^{k, \lambda}$ кривых $\gamma$ класса $C^{k+1, \lambda}$, для которых справедливо условие (5.2).

С инженерной точки зрения иногда разумно фиксировать также допустимый диапазон $[m, M]$ значений функции $N$ :

$$
m \leqslant N(s) \leqslant M \quad \forall P_{s} \in \gamma \quad(N(0)=N(|\gamma|)=0 \in[m, M]) .
$$

Соответствующий ограничению (5.3) подкласс кривых из множества $\mathscr{G}^{k, \lambda}$ можно было бы обозначить через $\mathscr{G}=\mathscr{G}^{k, \lambda}(m, M)$. Однако доказательства приводимых ниже теорем не зависят от того, фиксирован или нет диапазон $[m, M]$. 
Поэтому для краткости формулировок класс $\mathscr{G}=\mathscr{G}^{k, \lambda}(m, M)$ будем в дальнейшем обозначать также через $\mathscr{G}=\mathscr{G}^{k, \lambda}$.

Пусть функция $B: Q \ni w \mapsto B(u, v) \in \mathbb{R}$ есть решение задачи

$$
\Delta B=0 \quad \text { в } Q, \quad B(u, \pm 1)=0, \quad B( \pm \mu, v)=\beta( \pm v) .
$$

Здесь $\beta:[-1,1] \ni v \mapsto \beta(v)$, где

$$
\beta(v)=N(s(v)), \quad s:[-1,1] \ni v \mapsto s(v)=\int_{-1}^{v} e^{\alpha(\eta)} d \eta, \quad \alpha(v)=A(\mu, v),
$$

а функция $A$ гармонически-сопряжена к $B$. Функция $A$ определена с точностью до аддитивной константы. Рассмотрим отображение

$$
z: Q \ni w=u+i v \mapsto z(w)=\int_{\mu-i}^{w} \exp \{A(\xi, \eta)+i B(\xi, \eta)\} d \zeta \in \Omega_{\gamma}
$$

и определяемую им кривую

$$
z(\mu, \cdot):[-1,1] \ni v \mapsto z(\mu, v)=i \int_{-1}^{v} \exp \{\alpha(\eta)+i \beta(\eta)\} d \eta .
$$

Лемма 5.1. Кривая (5.7) принадлежит классу допустимых кривых $\mathscr{G}^{k, \lambda}$ тогда и толъко тогда, когда $F_{1}(\alpha, \beta)=0, F_{2}(\alpha, \beta)=0$, где

$$
F_{1}(\alpha, \beta)=\rho-\int_{-1}^{1} e^{\alpha(v)} \cos \beta(v) d v, \quad F_{2}(\alpha, \beta)=1+\int_{-1}^{1} e^{\alpha(v)} \sin \beta(v) d v,
$$

а $\rho$ - ордината точки $M_{1}(1, \rho)$ (см. рис. 11).

Действительно, нужно лишь проверить, что конец $z(\mu, 1)$ кривой $(5.7)$ имеет координаты $(1, \rho)$. Но именно это свойство выражают формулы (5.8).

Предположим, что существует кривая $\hat{\gamma}$, на которой достигается минимум функционала (5.1). Обозначим через $\hat{\alpha}$ и $\hat{\beta}$ те функции $\alpha$ и $\beta$ (определенные формулами (5.5)), которым соответствует эта кривая $\hat{\gamma}$.

TЕорема 5.1 [91]. Функиии $\hat{\alpha}$ и $\hat{\beta}$ дают решение задачи

$$
F_{0}(\alpha) \rightarrow \inf , \quad F_{1}(\alpha, \beta)=0, \quad F_{2}(\alpha, \beta)=0,
$$

¿əe

$$
F_{0}(\alpha)=\alpha^{+}-\alpha^{-}, \quad \alpha^{+}=\max _{|v| \leqslant 1} \alpha(v), \quad \alpha^{-}=\min _{|v| \leqslant 1} \alpha(v) .
$$

Действительно, обозначим $\operatorname{Im} w\left(P_{s}\right)$ через $v_{s}$, т. е. $w\left(P_{s}\right)=\mu+i v_{s}$. Имеем

$$
\left|\nabla u\left(P_{s}\right)\right|=\left.\left|\frac{d z}{d w}\right|_{w=\mu+i v_{s}}\right|^{-1} \text {. }
$$

Тем самым, $\ln \left|\nabla u\left(P_{s}\right)\right|=-\alpha\left(v_{s}\right)$. Поэтому

$$
\ln \left[\max _{P \in \gamma}|\nabla u(P)|\left(\min _{P \in \gamma}|\nabla u(P)|\right)^{-1}\right]=\max _{|v| \leqslant 1}(-\alpha(v))-\min _{|v| \leqslant 1}(-\alpha(v))=-\alpha^{-}+\alpha^{+} .
$$

Справедлива следующая теорема. 
Теорема 5.2 [91]. $1^{\circ}$. При любом целом $k \geqslant 0$ и любых числах $L>0$ и $\lambda \in(0,1)$ функиионал $\Phi$, определенный формулой (5.1), достигает минимума на множестве

$$
\mathscr{G}_{L}^{k, \lambda}=\left\{\gamma \in \mathscr{G}^{k, \lambda} \mid\left[N^{(k)}\right]_{\lambda} \leqslant L\right\}
$$

если

$$
\max N-\min N \leqslant \pi .
$$

Соответствующая кривая $\hat{\gamma}$ определяется формулой (5.7) по решению $(\hat{\alpha}, \hat{\beta})$ задачи (5.9).

$2^{\circ}$. Если функция $\hat{\beta}$ (она зависит от параметра $\mu=\left.u\right|_{\gamma}$ ) такова, что

$$
K(\mu) \stackrel{\text { def }}{=}\|\hat{\beta}\|_{C^{4}}<\infty \quad n p u \mu>0,
$$

то существуют константа $C$ и функиия $\mu \mapsto C(\mu)$, удовлетворяющая условию $|C(\mu)| \leqslant C K(\mu)$, такие, что

$$
\Phi(\hat{\gamma})=\inf _{\gamma \in G} \Phi(\gamma)=\exp \left\{\hat{\alpha}^{+}-\hat{\alpha}^{-}\right\}
$$

где $\hat{\alpha}^{+}=\max _{|v| \leqslant 1} \hat{\alpha}(v), \hat{\alpha}^{-}=\min _{|v| \leqslant 1} \hat{\alpha}(v)$, при этом

$$
\Phi(\hat{\gamma}) \longrightarrow \exp \left(\frac{C_{-1}}{\mu}+C_{0}+C_{1} \mu+C(\mu) \mu^{2}\right), \quad \text { nрu } \mu \rightarrow 0,
$$

əəe

$$
C_{-1}=\max _{|v| \leqslant 1} D_{-1}(v)-\min _{|v| \leqslant 1} D_{-1}(v), \quad D_{-1}(v)=\frac{1}{2} \int_{-1}^{v}(\hat{\beta}(-\eta)-\hat{\beta}(\eta)) d \eta .
$$

Если к тому же функция $\hat{\beta}$ еще и четна, то

$$
C_{-1}=C_{0}=0, \quad C_{1}(v)=\max _{|v| \leqslant 1} \hat{\beta}^{\prime}(v)-\min _{|v| \leqslant 1} \hat{\beta}^{\prime}(v) .
$$

Некоторые открытые вопросы. 1. Пусть функция $N$ имеет конечное число локальных экстремумов. Достигается ли в этом случае минимум функционала (5.1) на кривых класса $C^{1, \lambda}$ ?

2. Пусть кривая $\hat{\gamma}$ доставляет минимум функционалу (5.1) на кривых класса $C^{1, \lambda}$. При каких условиях на функцию $N$ можно утверждать, что эта кривая принадлежит классу $C^{1, \sigma}$ с $\sigma \in(\lambda, 1)$ ?

\section{§ 6. О выборе оптимальной скорости продвижения при обтекании по схеме Фёппля-Лаврентьева и об оптимизации динамики формы препятствия}

6.1. Пусть $\mathfrak{S} \subset \mathbb{R}^{2} \simeq \mathbb{C}$ - симметричное относительно оси $x$ препятствие для плоского потока жидкости единичной плотности, а $\vec{V}$ и $p$ - это соответственно скорость и давление, которые подчинены нестационарному гидродинамическому уравнению Эйлера

$$
\frac{d \vec{V}(t ; z(t))}{d t}=-\left.\nabla_{x, y} p(t, z)\right|_{z=z(t)}, \quad \vec{V}(t ; z(t)) \stackrel{\text { def }}{=} \frac{d}{d t} z(t),
$$


и уравнению неразрывности

$$
\operatorname{div} \vec{V}=0
$$

в искомой области течения $\widetilde{\Omega}(t)=\mathbb{C} \backslash\left\{\mathfrak{S} \cup z^{*}(t) \cup \bar{z}^{*}(t)\right\}$. Здесь

$$
z^{*}(t)=x^{*}(t)+i y^{*}(t) \quad \text { и } \quad \bar{z}^{*}(t)=x^{*}(t)-i y^{*}(t), \quad \text { где } y^{*}(t)>0,
$$

есть искомые центры двух точечных вихрей в следе при обтекании препятствия $\mathfrak{S}$, вне которых скорость течения имеет потенциал $u$ :

$$
\vec{V}(t ; x+i y)=\nabla_{x, y} u(t ; x, y) \quad \text { для }(x, y) \in \widetilde{\Omega}(t) .
$$

Через $z(t)=x(t)+i y(t) \in \mathbb{C}$ в (6.1) и ниже обозначен тот центр элементарного объема (иначе говоря, частицы) жидкости, который при $t=0$ находился в точке $z(0)=x(0)+i y(0)$. Интенсивность вихрей считается известной, иными словами, в асимптотической формуле

$$
\vec{V}(t ; x+i y) \sim \frac{\omega}{2 \pi} \frac{\left(\left(y-y^{*}\right),-\left(x-x^{*}\right)\right)}{\left|z-z^{*}\right|^{2}} \quad \text { при } z \rightarrow z^{*}
$$

считается известным коэффициент $\omega>0$. Скорость потока на бесконечно-

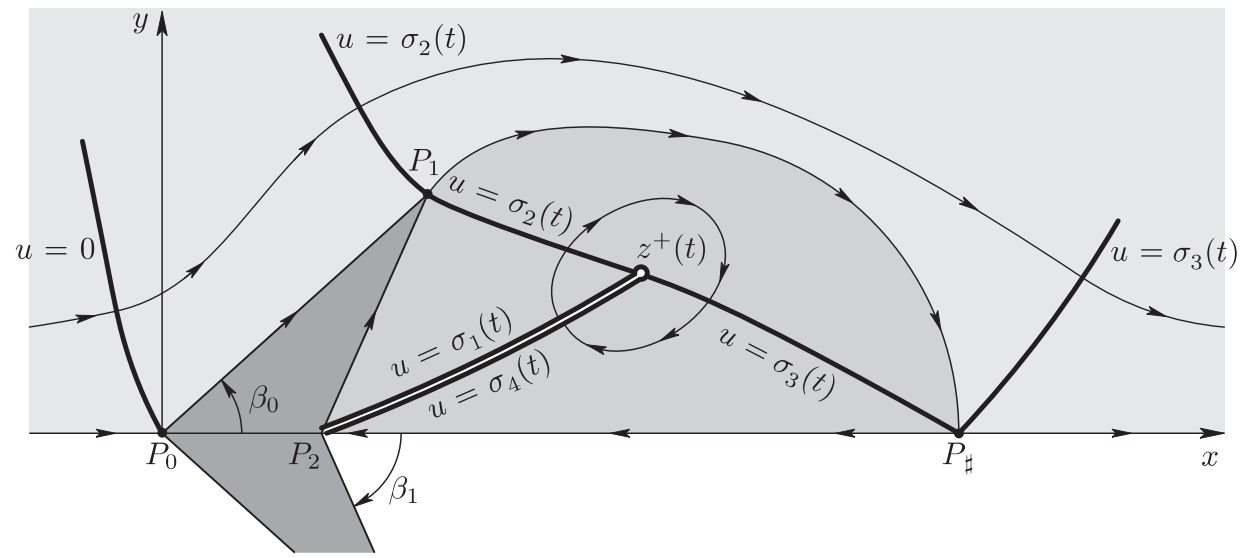

Рис. 12

сти $\mathbf{V}: t \mapsto \mathbf{V}(t)$ (или, в эквивалентной интерпретации, скорость продвижения объекта $\mathfrak{S}$ в неподвижной на бесконечности жидкости) является варьируемым параметром. Эта величина подчинена условиям

$$
0<V_{0}(t) \leqslant \mathbf{V}(t) \leqslant V_{1}(t)
$$

где функции $V_{0}$ и $V_{1}$ заданы, причем

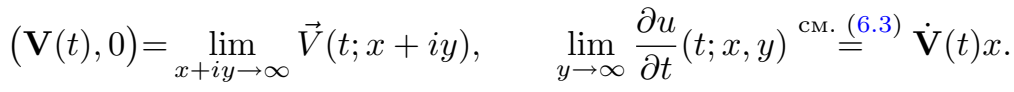

Известным считается также давление на бесконечности

$$
\left.p\right|_{\infty} \stackrel{\text { def }}{=} \lim _{x+i y \rightarrow \infty} p(t, x+i y) .
$$


Относительно препятствия $\mathfrak{S}$ предполагается, что оно фиксировано и непроницаемо, а его граница Г есть ломаная. При этом для большей простоты изложения мы ограничимся здесь лишь базовым случаем, в котором Г есть ломаная, соединяющая вершины четырехугольника (см. рис. 12). Три из них: $P_{0}, P_{1}$ и $P_{2}$ представлены на рисунке. Четвертая вершина $\bar{P}_{1}$ (она на рисунке не отмечена) симметрична точке $P_{1}$ относительно оси $x$. Уголок $\Gamma_{0}=P_{0} P_{2} \cup P_{0} \bar{P}_{1}$ омывается набегающим потоком, а к уголку $\Gamma_{1}=P_{1} P_{2} \cup P_{1} \bar{P}_{1}$, который составляет кормовую часть препятствия, примыкает вихревой след.

Управляющий параметр $\mathbf{V}(t) \in\left[V_{0}(t), V_{1}(t)\right]$ влияет на скорость $\vec{V}$ и давление $p$, а тем самым, на целевую функцию в той или иной задаче оптимального управления. Нас будут интересовать те задачи оптимального управления, которые связаны с частицами жидкости $z_{0}(t)=x_{0}(t)+i y_{0}(t)$, которые скользят или будут скользить вдоль препятствия в рассматриваемый период времени.

Вот два наиболее простых, но отнюдь не тривиальных примера таких задач.

Задача быстродействия. Требуется минимизировать время $T>0$ при условии, что

$$
x_{0}(-T)-x_{0}(0)=-1 \quad \Longleftrightarrow \quad \int_{-T}^{0} \operatorname{Re} \vec{V}\left(t ; x_{0}(t)\right) d t=1,
$$

т. е. речь идет о выборе в каждый момент времени скорости $\mathbf{V}(t)$ продвижения объекта в стоячей воде, при которой дистанция единичной длины будет пройдена за кратчайшее время при наличии в следе двух точечных вихрей известной интенсивности.

Задача о минимуме лобового сопротивления. Требуется при заданном $T>0$ минимизировать интеграл

$$
\int_{0}^{T}\left(\int_{\Gamma_{0}} p(t, s) d s-\int_{\Gamma_{1}} p(t, s) d s\right) d t
$$

в частности, когда $\max _{0 \leqslant t \leqslant T} V_{0}(t)>\min _{0 \leqslant t \leqslant T} V_{1}(t)$.

Основная трудность в изучении такого рода задач, т. е. задач следующего вида:

$$
F(p, \vec{V}) \rightarrow \text { inf, } \quad G(p, \vec{V})=0,
$$

связана с тем, что фигурирующие в (6.7) величины $p$ и $\vec{V}$ зависят от параметра управления $\mathbf{V}$ очень опосредованно, а именно через решение задачи со свободной границей $z^{*}(t) \cup \bar{z}^{*}(t)$ (состоящей из искомых вихревых центров) для нестационарного гидродинамического уравнения Эйлера (6.1) и уравнения неразрывности (6.2).

Возможно ли для функций $s \mapsto p(t, s)$ и $s \mapsto \vec{V}(t ; s)$, входящих в функционалы (6.7), выразить их зависимость от управляющего параметра $\mathbf{V}$ более явно, не прибегая к непосредственному решению сложной задачи (6.1)-(6.6) со свободной границей для нестационарной нелинейной системы уравнений с частными производными?

Ниже дан положительный ответ на этот вопрос.

6.2. Отметим, что рассматриваемая здесь схема обтекания была в стационарном случае (т. е. при $\mathbf{V}=$ const) предложена Л. Фёпплем [92] и развита M. А. Лаврентьевым (см., например, [93]). Вслед за классиками проигнорируем наличие тангенциального разрыва скорости на кривой $\gamma(t)$, отделяющей 
вихревой след от “ламинарного" потока, огибающего препятствие. При этом определим свободную границу $\gamma(t)$ как ту кривую, проходящую через "крайние" точки препятствия $P_{2}$ и $\bar{P}_{2}$, в каждой точке $z=x+i y \in \gamma(t)$ которой вектор $\vec{V}(t ; z)$ является касательным к ней. Такая линия называется линией тока. Следует иметь в виду, что лишь в стационарном случае линия тока совпадает с траекторией частицы жидкости. Выбранный здесь (в отличие от случая кавитационного обтекания [94]) упрощающий реальную ситуацию подход с выбором кривой $\gamma(t)$ оправдывается тем, что конфигурация следа за препятствием слабо [95] влияет на распределение давления и скорость вблизи препятствия, а потому и на функционалы оптимизационной задачи (6.7). Будем предполагать также, что при каждом $t$ функция

$$
\Gamma \ni x+i y \mapsto u(t ; x, y)
$$

кусочно непрерывно-дифференцируема и обратима.

Выделим в верхней части $\widetilde{\Omega}(t) \cap \mathbb{R}_{+}^{2}$ области течения односвязную область $\Omega_{t}=\left(\widetilde{\Omega}(t) \cap \mathbb{R}_{+}^{2}\right) \backslash \gamma^{*}(t)$, где $\gamma^{*}(t)$ - та (представленная на рис. 12 двойной линией) кривая, которая выходит из точки $P_{1}$ и идет до искомого вихревого центра $z^{*}(t)$ вдоль эквипотенциальной линии, т. е. вдоль линии уровня потенциала $u(t ; \cdot, \cdot)$. Для краткости будем называть эквипотенциальную линию эквипотенциалъю. Кривая $\gamma^{*}(t)$ разрезает двусвязную область $\widetilde{\Omega} \cap \mathbb{R}_{+}^{2}$. Берега разреза суть эквипотенциали. Значения потенциала на них обозначим через $\sigma_{1}(t)$ и $\sigma_{4}(t)\left(\sigma_{1}<\sigma_{4}\right)$. А через $\sigma_{2}(t)$ и $\sigma_{3}(t)$ обозначим те значения потенциала $u(t ; \cdot, \cdot)$, которые задают эквипотенциали, проходящие соответственно через точку $P_{2}$ срыва с препятствия потока и лежащую на оси $x$ точку $P_{3} \in \gamma(t)$. При этом для каждого конкретного $t$ соотнесем нулевое значение потенциала $u(t ; \cdot, \cdot)$ с точкой $P_{0}$ (носовой кромкой препятствия, помещенной в начало координат). Отметим, что

$$
\sigma_{1}(t)<\sigma_{2}(t)<\sigma_{3}(t)<\sigma_{4}(t)=\sigma_{1}(t)+\omega .
$$

В области $\Omega_{t}$ введем функцию $v$, гармонически-сопряженную к функции $u$, зафиксировав равенством

$$
w\left(t, P_{0}\right)=0
$$

аддитивную константу аналитического отображения

$$
w(t, \cdot): \Omega_{t} \ni z=x+i y \mapsto w(t, z)=u(t ; x, y)+i v(t ; x, y) .
$$

Это отображение, в силу условия $\nabla u \neq 0$ и сделанного выше предположения о функции (6.8), однолистно и непрерывно вплоть до $\partial \Omega_{t}=\partial \Omega_{t}^{+} \cup \partial \Omega_{t}^{-} \backslash \lambda_{t}$, где $\Omega_{t}^{+}$и $\Omega_{t}^{-}-$это определяемые ниже две зоны течения: ламинарная (на рис. 12 она наиболее светлая) и вихревая, а

$$
\lambda_{t}=\left\{(x, y) \in \mathbb{R}_{+}^{2} \mid \sigma_{2}(t)<u(t ; x, y)<\sigma_{3}(t), v(t ; x, y)=0\right\}
$$

есть та дуга нулевой линии тока $\left\{(x, y) \in \mathbb{R}^{2} \mid v(t ; x, y)=0\right\}$, вдоль которой склеиваются обе зоны течения. Нулевая линия тока идет по мере роста потенциала от $-\infty$ до $+\infty$ сначала вдоль оси $x$ (в силу условия симметрии) от $-\infty$ до точки $P_{0}$, затем вдоль отрезка $P_{0} P_{2}$ (передней части непроницаемого препятствия). Далее, сорвавшись с препятствия, она дугой $\lambda_{t}$ отделяет вихревую зону 
течения $\Omega_{t}^{-}$от ее ламинарной части $\Omega_{t}^{+}$. В области $\Omega_{t}^{+}$функция $v$ монотонно возрастает от 0 до $\infty$ вдоль любой эквипотенциали $\{u(x, y)=$ const $\in(-\infty, \infty)\}$ по мере удаления от нулевой линии тока. Поэтому ламинарная область течения $\Omega_{t}^{+}$отображается функцией $w=u+i v$ на верхнюю полуплоскость $\mathbb{C}_{+}$. В области $\Omega_{t}^{-}$функция $v$ монотонно убывает от 0 до $-\infty$ вдоль любой эквипотенциали $\left\{u(x, y)=\right.$ const $\left.\in\left(\sigma_{1}, \sigma_{4}\right)\right\}$ по мере приближения к вихревому центру $z^{*}(t)$. Поэтому функция $w=u+i v$ отображает вихревую область течения $\Omega_{t}^{-}$на полуполосу

$$
\Pi(t)=\left\{\sigma_{1}(t)<u<\sigma_{4}(t), v<0\right\} .
$$

6.3. Область $w\left(\Omega_{t}\right)$ будем обозначать через $Q(t)$. Как было установлено,

$$
Q(t)=\mathbb{C}_{+} \cup \mathrm{I}(t) \cup \Pi(t),
$$

где $\mathrm{I}(t) \stackrel{(6.10)}{=} w\left(\lambda_{t}\right)$, т. е. $\mathrm{I}(t)=\left\{\sigma_{2}(t)<u<\sigma_{3}(t), v=0\right\}$ - тот интервал, который соединяет полуплоскость $\mathbb{C}_{+}$с полуполосой $\Pi(t)$. Введем в $Q=Q(t)$ функцию Гельмгольца-Кирхгофа

$$
A+i B: Q(t) \ni w=u+i v \mapsto A(u, v)+i B(u, v) \stackrel{\text { def }}{=} \ln \frac{d z}{d w} .
$$

Эта функция определена в области $Q=Q_{\sigma(t)}$, которая параметризована $u c$ комой вектор-функцией $\sigma=\left(\sigma_{1}, \sigma_{2}, \sigma_{3}\right)$. Напомним, что $\sigma_{4}=\sigma_{1}+\omega$, где $\omega-$ заданный параметр. Координаты вершин препятствия

$$
P_{0}=(0,0), \quad P_{1}=\left(x_{1}, 0\right), \quad P_{2}=\left(x_{2}, y_{2}\right), \quad \text { где } x_{1}>0, x_{2} \in \mathbb{R}, y_{2}>0,
$$

заданы. Тем самым, известны углы $\beta_{0} \in(0, \pi)$ и $\beta_{1} \in\left(\beta_{0}, \pi\right)$ наклона отрезков $P_{0} P_{1}$ и $P_{1} P_{2}$ к оси $x$, а именно:

$$
\operatorname{tg} \beta_{0}=\frac{y_{2}}{x_{2}}, \quad \operatorname{tg} \beta_{1}=\frac{y_{2}}{x_{2}-x_{1}} .
$$

Таким образом, функция $A+i B=A^{\sigma, \beta}+i B^{\sigma, \beta}$ параметрически зависит от $\sigma(t)=\left(\sigma_{1}(t), \sigma_{2}(t), \sigma_{3}(t)\right)$ и $\beta=\left(\beta_{0}, \beta_{1}\right)$. Так как $A+i B=\ln (d z / d w)$, а $w(0)=0$, то

$$
z(w)=\int_{0}^{w} e^{A(\xi, \eta)+i B(\xi, \eta)} d \zeta, \quad \text { где } \zeta=\xi+i \eta \in Q(t) .
$$

Поэтому для нахождения вектор-функции $\sigma=\left(\sigma_{1}, \sigma_{2}, \sigma_{3}\right)$ получаем систему из трех уравнений

$$
\begin{gathered}
x_{1}=\operatorname{Re}\left[\int_{+i 0}^{\sigma_{2}+i 0} \mathscr{E}\left(\zeta ; x_{1}, y_{1}, x_{2}\right) d \zeta-\int_{\sigma_{1}-i 0}^{\sigma_{2}-i 0} \mathscr{E}\left(\zeta ; x_{1}, y_{1}, x_{2}\right) d \zeta\right] \\
x_{2}=\operatorname{Re} \int_{+i 0}^{\sigma_{2}+i 0} \mathscr{E}\left(\zeta ; x_{1}, y_{1}, x_{2}\right) d \zeta, \quad y_{2}=\operatorname{Im} \int_{+i 0}^{\sigma_{2}+i 0} \mathscr{E}\left(\zeta ; x_{1}, y_{1}, x_{2}\right) d \zeta,
\end{gathered}
$$

где

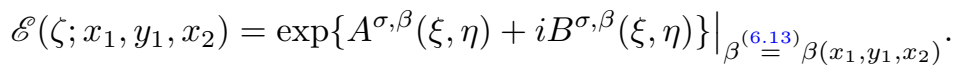


Входящая в эту систему функция $A+i B$ строится по гармонической функции $B: Q \rightarrow \mathbb{R}$, удовлетворяющей, как можно проверить [96], следующим граничным условиям:

$$
\begin{aligned}
B(u,+0) & =\beta_{0} \quad \text { при } u \in\left(0, \sigma_{2}\right), & B(u,+0) & =0 \quad \text { при } u \notin\left[0, \sigma_{2}\right], \\
B(u,-0) & =\beta_{1} \quad \text { при } u \in\left(\sigma_{1}, \sigma_{2}\right), & B(u,-0) & =-\pi \quad \text { при } u \in\left(\sigma_{3}, \sigma_{4}\right), \\
B\left(\sigma_{1}, v\right) & =B\left(\sigma_{4}, v\right)+2 \pi, & \partial_{u} B\left(\sigma_{1}, v\right) & =\partial_{u} B\left(\sigma_{4}, v\right) \quad \text { при } v<0 .
\end{aligned}
$$

Теорема 6.1 [96]. В области $Q=\mathbb{C}_{+} \cup \mathrm{I} \cup \Pi$ существует одна и только одна ограниченная гармоническая функиия $B: Q \rightarrow \mathbb{R}$, удовлетворяющая краевым условиям (6.15)-(6.17).

ДокАзАтельство. Достаточно показать, что краевая задача (6.15)-(6.17) в области $Q$ для гармонической функции $B$ эквивалентна задаче Дирихле для уравнения Пуассона в круге:

$$
\Delta \Psi=f \quad \text { в } \mathscr{D}=\{\tau \in \mathbb{C}|| \tau \mid<1\}, \quad \Psi=g \quad \text { на } \partial \mathscr{D} .
$$

Здесь $f \in C^{\infty}(\overline{\mathscr{D}})$, а $g$ - кусочно гладкая функция на границе $\partial \mathscr{D}$ круга $\mathscr{D}$. При этом $B(u, v)=\Psi(\tau(w))$, где

$$
\tau: \bar{Q} \ni w=u+i v \mapsto \tau(w) \in \overline{\mathscr{D}}=\{\tau \in \mathbb{C}|| \tau \mid \leqslant 1\}
$$

есть гомеоморфизм, диффеоморфно отображающий $Q$ на $\mathscr{D}$.

Отображение $\tau$ построим в виде композиции $\mu \circ \zeta$ двух определяемых ниже отображений.

Пусть $\Lambda=\left\{\sigma_{1}<u<\sigma_{4}, v \in \mathbb{R}\right\}$ - полоса ширины $2 h=\sigma_{4}-\sigma_{1}$, симметричная относительно оси $\{u=a\}$, где $a=\sigma_{1}+h$. Полагая

$$
D_{ \pm}=D \cap \mathbb{C}_{ \pm}, \quad \text { где } D=\left\{\xi^{2}+\eta^{2}<1\right\}, \text { а } \mathbb{C}_{ \pm}=\{\xi \pm i \eta \in \mathbb{C} \mid \eta>0\},
$$

введем конформные отображения

$$
S: \Lambda \rightarrow D=\left\{\xi^{2}+\eta^{2}<1\right\} \quad \text { и } \quad T: \mathbb{C}_{+} \rightarrow D_{+}
$$

следующими формулами:

$$
\begin{gathered}
S(w)=i \frac{1-p(w)}{1+p(w)}, \quad p(w)=e^{q(w)}, \quad q(w)=i(w-a) \frac{\pi}{2 h}, \quad \text { где } w \in \Lambda, \\
T(w)=\frac{1+i \sqrt{(w-a)^{2}-1}}{w-a}, \quad \text { где } w \in \mathbb{C}_{+} .
\end{gathered}
$$

Через $\varphi: Q \stackrel{(6.12)}{=} \mathbb{C}_{+} \cup \mathrm{I} \cup \Pi \rightarrow[0,1]$ обозначим гладкую функцию такую, что

$$
\varphi(u+i v)= \begin{cases}1, & \text { если }|u-a|<h \text { и } v<\sqrt{h^{2}-(u-a)^{2}}, \\ 0, & \text { если } v>0 \text { и }(u-a)^{2}+v^{2}>(2 h)^{2} .\end{cases}
$$

Используя введенные выше обозначения

$$
\begin{array}{rlrl}
\Pi & =\left\{\sigma_{1}<u<\sigma_{4}, v<0\right\}, \quad \mathrm{I} & =\left\{\sigma_{2}<u<\sigma_{3}, v=0\right\}, \\
D_{ \pm} & =\mathbb{C}_{ \pm} \cap\left\{\xi^{2}+\eta^{2}<1\right\}, & \mathbb{C}_{ \pm} & =\{\xi \pm i \eta \in \mathbb{C}, \eta>0\},
\end{array}
$$


зададим формулой

$$
\zeta(w)= \begin{cases}S(w), & w \in \Pi \cup I, \\ (1-\varphi(w)) T(w)+\varphi(w) S(w), & w \in I \cup \mathbb{C}_{+},\end{cases}
$$

отображение

$$
\zeta: Q \ni w=u+i v \mapsto \zeta(w)=\xi(w)+i \eta(w) \in P \stackrel{\text { def }}{=} \zeta(Q) .
$$

Имеем: $P=D_{-} \cup\left\{\xi_{2}<\xi<\xi_{3}, \eta=0\right\} \cup D_{+}$. При этом

$$
-1=\zeta\left(\sigma_{1}, 0\right)<\xi_{2} \stackrel{\text { def }}{=} \zeta\left(\sigma_{2}, 0\right)<\xi_{3} \stackrel{\text { def }}{=} \zeta\left(\sigma_{3}, 0\right)<\zeta\left(\sigma_{4}, 0\right)=1 .
$$

Введем функцию

$$
U: P \ni \zeta=\rho e^{i \theta} \mapsto U(\rho, \theta)=B(w(\zeta))+2 \pi \varphi(w(\zeta)) \frac{u(\zeta)-\sigma_{1}}{\sigma_{4}-\sigma_{1}} .
$$

Имеем

$$
\begin{array}{rlrl}
\Delta U=F \text { в } P, U(1, \theta) & =g_{0}(\theta) & & \text { при } \theta \in(0, \pi), \\
U(\rho,+i 0) & =g_{1}(\rho) & & \text { при } \zeta\left(\sigma_{3}+i 0\right)<\rho<\zeta\left(\sigma_{4}+i 0\right), \\
U(\rho,-i 0) & =g_{2}(\rho) & & \text { при } \zeta\left(\sigma_{3}-i 0\right)<\rho<\zeta\left(\sigma_{4}-i 0\right), \\
U(\rho, \pi-0) & =g_{3}(\rho) & & \text { при } \zeta\left(\sigma_{1}+i 0\right)<\rho<\zeta\left(\sigma_{2}+i 0\right), \\
U(\rho,-\pi+0) & =g_{4}(\rho) & \text { при } \zeta\left(\sigma_{1}-i 0\right)<\rho<\zeta\left(\sigma_{2}-i 0\right), \\
U\left(\rho,-\frac{\pi}{2}+\theta\right) & =U\left(\rho,-\frac{\pi}{2}-\theta\right) & \text { при } \theta \in(0, \pi / 2), \\
-\left.\partial_{\rho} U\left(\rho,-\frac{\pi}{2}+\theta\right)\right|_{\rho=1} & =\left.\partial_{\rho} U\left(\rho,-\frac{\pi}{2}-\theta\right)\right|_{\rho=1} & & \text { при } \theta \in(0, \pi / 2),
\end{array}
$$

где функции $g_{0}, g_{1}, g_{2}, g_{3}, g_{4}$ кусочно непрерывны, а $F \in C^{\infty}(\bar{P})$.

В качестве функции $\mu$ в искомой композиции $\tau=\mu \circ \zeta: Q \stackrel{(6.19)}{\rightarrow} \mathscr{D}$ возьмем однолистное отображение $P$ на $\mathscr{D}$, подчиненное такому условию: граничные дуги

$$
\Gamma_{+}=\left\{\zeta=e^{i \theta} \mid-\pi / 2<\theta<0\right\}, \quad \Gamma_{-}=\left\{\zeta=e^{i \theta} \mid-\pi<\theta<-\pi / 2\right\}
$$

полудиска $D_{-} \subset P$ отображаются в берега разреза круга $\mathscr{D}$ вдоль его радиуса, причем точка $(\rho, \theta)=(1,-\pi / 2) \in \partial P$ трансформируется в центр круга $\mathscr{D}$.

В силу последних двух условий в (6.23), функция $\Psi=U \circ \zeta: \mu \mapsto \Psi(\mu)$ удовлетворяет уравнению $\Delta \Psi=F \circ \zeta$ в $\mathscr{D} \backslash\{0\}=\{0<|\mu|<1\} \subset \mathscr{D}$. Поскольку функция $\Psi$ ограничена (ибо, по условию, ограничена функция $U$ ), то, ввиду теоремы об устранимой особенности, функция $\Psi$ удовлетворяет во всем круге $\mathscr{D}$ уравнению $\Delta \Psi=F \circ \zeta$, т. е. уравнению (6.18) с $f=F \circ \zeta$. Теорема 6.1 доказана.

Формулы для функции $B$ приведены в [96]. Функция $A$, гармонически-сопряженная к функции $B$, восстанавливается по $B$ однозначно по приведенным в [96] формулам с учетом того, что $A(u, v) \rightarrow-\ln |\vec{V}(t ; \infty)|$ при $u \rightarrow \infty$, ибо

$$
\vec{V}=\nabla u \stackrel{(6.9)}{=} \frac{\overline{d w}}{d z}=\overline{\frac{1}{d z / d w}} \stackrel{(6.14)}{=} e^{-A} e^{i B} .
$$


6.4. Ввиду (6.24) и соотношения $d s=e^{A(u, 0)} d u$, для представления интегральных функционалов задачи (6.7) в виде функционалов классического вариационного исчисления остается лишь выписать формулы, которые бы позволили выразить давление $p$ через относительную скорость $\mathbf{V}(t)$. Этому посвящены следующие три леммы, в которых фигурирует обозначение

$$
w(t)=u(t)+i v(t) \stackrel{\text { def }}{=} w(t ; z(t)),
$$

т. е. $w(t)$ есть образ траектории $t \mapsto z(t)$ при отображении $w: \Omega \rightarrow Q$.

Лемма 6.1. Справедливо равенство

$$
\frac{d u(t)}{d t}=\dot{u}(t)+e^{-2 A(u(t), v(t))}, \quad \text { гдe }\left.\dot{u}(t) \stackrel{\text { def }}{=} \frac{\partial u(t ; x, y)}{\partial t}\right|_{x=x(t), y=y(t)} .
$$

ДокАЗАТЕЛЬСтво. Имеем:

$$
\frac{d u(t)}{d t}=\dot{u}(t)+\left.\left(\frac{\partial u(t ; x, y)}{\partial x} \frac{d x(t)}{d t}+\frac{\partial u(t ; x, y)}{\partial y} \frac{d y(t)}{d t}\right)\right|_{x=x(t), y=y(t)}
$$

a

$$
\left(\frac{d x(t)}{d t}, \frac{d y(t)}{d t}\right) \stackrel{(6.1)}{=} \vec{V}(t ; z(t))=\left.\left(\frac{\partial u(t ; x, y)}{\partial x}, \frac{\partial u(t ; x, y)}{\partial y}\right)\right|_{x=x(t), y=y(t)}
$$

Поэтому

$$
\frac{d u(t)}{d t}=\dot{u}(t)+|\vec{V}(t ; z(t))|^{2} \stackrel{(6.24)}{=} \dot{u}(t)+e^{-2 A(u(t), v(t))},
$$

что и доказывает лемму.

Лемма 6.2. Давление $p(t, \cdot)$ в точке $z(t)=x(t)+i y(t) \in \Omega(t)$ может быть въчислено по формуле

$$
p(t, z(t))=\left.p\right|_{\infty}+\frac{|\vec{V}(t ; z(t))|^{2}+\mathbf{V}^{2}(t)}{2}+\dot{\mathbf{V}}(t) x(t)-\frac{d u(t ; x(t), y(t))}{d t} .
$$

ДокАзАтЕльство. Уравнение Эйлера (6.1) имеет интеграл Коши-Лагранжа [97]

$$
p(t, x+i y)+\frac{1}{2}|\vec{V}(t ; x+i y)|^{2}+\frac{\partial u}{\partial t}(t ; x, y)=p_{0}(t) .
$$

Поэтому

$$
\left.p_{0}(t) \stackrel{(6.5),(6.6)}{=} p\right|_{\infty}+\frac{1}{2} \mathbf{V}^{2}(t)+\dot{\mathbf{V}}(t) x(t) .
$$

Учитывая (6.28) и (6.31), имеем

$p(t, x(t)+i y(t)) \stackrel{(6.30)}{=} p_{0}-\frac{1}{2}|\vec{V}|^{2}-\dot{u}=\left.p\right|_{\infty}+\frac{1}{2} \mathbf{V}^{2}(t)+\dot{\mathbf{V}}(t) x(t)-\frac{|\vec{V}|^{2}}{2}+|\vec{V}|^{2}-\frac{d u}{d t}$, что и доказывает лемму.

Чтобы завершить построение формулы, выражающей давление через функцию $\mathbf{V}$, остается найти слагаемое $\frac{d u}{d t} \stackrel{\text { def }}{=} \frac{d}{d t} u(t ; x(t), y(t))$ в формуле (6.29). 
Лемма 6.3. Функиия $t \mapsto w(t)=u(t)+i v(t)$ есть решение системы обыкновенных дифберенциальных уравнений

$$
\frac{d u}{d t}=X_{0}(t, u, v ; \mathbf{V})+X_{1}(t, u, v ; \mathbf{V}) \dot{\mathbf{V}}, \quad \frac{d v}{d t}=Y_{0}(t, u, v ; \mathbf{V})+Y_{1}(t, u, v ; \mathbf{V}) \dot{\mathbf{V}}
$$

где искомый вектор $X=\left(X_{0}, X_{1}\right)$, а также вектор $Y=\left(Y_{0}, Y_{1}\right)$ определены в нижеследующих формулах (6.39) и (6.40).

ДокАЗАТЕЛЬство. Согласно (6.14), траектория $z: t \mapsto z(t)=x(t)+i y(t)$ центра элементарного объема жидкости вычисляется по формуле

$$
z(t)=\int_{0}^{w(t)} \exp \{A(\xi, \eta)+i B(\xi, \eta)\} d \zeta, \quad \zeta=\xi+i \eta,
$$

где $w(t) \stackrel{(6.25)}{=} u(t)+i v(t)$ есть образ $w(t, z(t))$ этой траектории при отображении $w$. Вводя обозначение

$$
[F]^{u, v} \stackrel{\text { def }}{=} F(u, v),
$$

сопоставим две формулы

$$
\vec{V}(t ; z(t)) \stackrel{(6.24)}{=}\left[e^{-A}(\cos B+i \sin B)\right]^{u(t), v(t)} \quad \text { и } \quad \vec{V}(t ; z(t)) \stackrel{(6.1)}{=} \frac{d}{d t} z(t),
$$

обратив внимание на то, что $z(t)$, согласно (6.33), выражается через функцию $A+i B$. Запишем интеграл в формуле (6.33) в виде суммы интегралов

$$
\int_{0+i 0}^{u_{*}+i 0}+\int_{u_{*}+i 0}^{u_{*}+i v(t)}+\int_{u_{*}+i v(t)}^{w(t)=u(t)+i v(t)}, \quad \text { где } u_{*} \in\left(\sigma_{2}, \sigma_{3}\right),
$$

и продифференцируем $z(t)$, учитывая, что величина $E(u, v)=e^{A(u, v)+i B(u, v)}$ параметрически зависит от $\sigma(t)=\left(\sigma_{1}(t), \sigma_{2}(t), \sigma_{3}(t)\right)$ и $\mathbf{V}(t)$. Получим

$$
\begin{aligned}
\vec{V}(t ; z(t)) \stackrel{(6.34)}{=} & \left\{\int_{u_{*}}^{u(t)} \frac{d}{d t}[E]^{\xi, v(t)} d \xi+\frac{d u}{d t}[E]^{u(t), v(t)}\right\} \\
& +i\left\{\int_{0}^{v(t)} \frac{d}{d t}[E]^{u_{*}, \eta} d \eta+\frac{d v}{d t}[E]^{u_{*}, v(t)}\right\} \\
= & \frac{d u}{d t}\left\{[E]^{u(t), v(t)}\right\}+\frac{d v}{d t}\left\{\int_{u_{*}}^{u(t)}\left[E_{v}\right]^{\xi, v(t)} d \xi+i[E]^{u_{*}, v(t)}\right\} \\
& +\sum_{k=1}^{3} \dot{\sigma_{k}}(t)\left\{\int_{u_{*}}^{u(t)}\left[E_{\sigma_{k}}\right]^{\xi, v(t)} d \xi+i \int_{0}^{v(t)}\left[E_{\sigma_{k}}\right]^{u_{*}, \eta} d \eta\right\} \\
& +\dot{\mathbf{V}}(t)\left\{\int_{u_{*}}^{u(t)}\left[E_{\mathbf{V}}\right]^{\xi, v(t)} d \xi+i \int_{0}^{v(t)}\left[E_{\mathbf{V}}\right]^{u_{*}, \eta} d \eta\right\} .
\end{aligned}
$$

Для сокращения записи будем символом $\rho_{k}$ обозначать $k$-ю компоненту вектора $\left(\sigma_{1}, \sigma_{2}, \sigma_{3}, \mathbf{V}\right)$, а через $\Phi_{q}$ будем обозначать производную той или иной функции $\Phi$, зависящей от переменной $q$. Полагая

$$
\cos \{A B\}_{q} \stackrel{\text { def }}{=}\left(A_{q} \cos B-B_{q} \sin B\right), \quad \sin \{A B\}_{q} \stackrel{\text { def }}{=}\left(A_{q} \sin B+B_{q} \cos B\right)
$$


и вспоминая, что $E=e^{A} \cos B+i e^{A} \sin B$, заметим, что

$$
E_{v}=e^{A+i B}\left(\frac{\partial A}{\partial v}+i \frac{\partial B}{\partial v}\right)
$$

Аналогичные формулы верны для $E_{\rho_{k}}$. Поэтому правая часть (6.37) приобретает вид

$$
\begin{aligned}
\frac{d u}{d t}\{ & {\left.\left[e^{A} \cos B+i e^{A} \sin B\right]^{u(t), v(t)}\right\} } \\
& +\frac{d v}{d t}\left\{\int_{u_{*}}^{u(t)}\left[E_{v}\right]^{\xi, v(t)} d \xi+i\left[e^{A} \cos B+i e^{A} \sin B\right]^{u_{*}, v(t)}\right\} \\
& +\sum_{k=1}^{4} \dot{\rho_{k}}(t)\left\{\int_{u_{*}}^{u(t)}\left[E_{\rho_{k}}\right]^{\xi, v(t)} d \xi+i \int_{0}^{v(t)}\left[E_{\rho_{k}}\right]^{u_{*}, \eta} d \eta\right\} .
\end{aligned}
$$

Преобразованную таким образом правую часть равенства (6.37) сопоставим с ее левой частью

$$
\vec{V}(t ; z(t)) \stackrel{(6.35)}{=}\left[e^{-A}(\cos B+i \sin B)\right]^{u(t), v(t)}
$$

и распишем раздельно вещественную и мнимую компоненты равенства (6.37). Тогда получим

$$
h_{1}=e^{11} \frac{d u}{d t}+e^{12} \frac{d v}{d t}+\sum_{k=1}^{4} f_{k}^{1 \rho} \frac{d \rho_{k}}{d t}, \quad h_{2}=e^{21} \frac{d u}{d t}+e^{22} \frac{d v}{d t}+\sum_{k=1}^{4} f_{k}^{2 \rho} \frac{d \rho_{k}}{d t},
$$

где

$$
\begin{aligned}
h_{1} & =\left[e^{-A} \cos B\right]^{u(t), v(t)}, & h_{2} & =\left[e^{-A} \sin B\right]^{u(t), v(t)}, \\
e^{11} & =\left[e^{A} \cos B\right]^{u(t), v(t)}, & e^{12} & =\int_{0}^{u(t)}\left[e^{A} \cos \{A B\}_{v}\right]^{\xi, v(t)} d \xi-\left[e^{A} \sin B\right]^{0, v(t)}, \\
e^{21} & =\left[e^{A} \sin B\right]^{u(t), v(t)}, & e^{22} & =\int_{0}^{u(t)}\left[e^{A} \sin \{A B\}_{v}\right]^{\xi, v(t)} d \xi+\left[e^{A} \cos B\right]^{0, v(t)},
\end{aligned}
$$

a

$$
\begin{aligned}
& f_{k}^{1 \rho}=\int_{0}^{u(t)}\left[e^{A} \cos \{A B\}_{\rho_{k}}\right]^{\xi, v(t)} d \xi-\int_{0}^{v(t)}\left[e^{A} \sin \{A B\}_{\rho_{k}}\right]^{0, \eta} d \eta, \\
& f_{k}^{2 \rho}=\int_{0}^{u(t)}\left[e^{A} \sin \{A B\}_{\rho_{k}}\right]^{\xi, v(t)} d \xi+\int_{0}^{v(t)}\left[e^{A} \cos \{A B\}_{\rho_{k}}\right]^{0, \eta} d \eta .
\end{aligned}
$$

Учитывая, что в каждый момент времени однозначно определен потенциал $u(t)=u(t, x(t), y(t))$ скорости движения $t \mapsto(x(t), y(t))$ элементарного объема жидкости, заключаем, что однозначно определена и скорость $d u / d t$ изменения этого потенциала. Поэтому детерминант $D \stackrel{\text { def }}{=} e^{11} e^{22}-e^{21} e^{12}$ системы (6.38) относительно $d u / d t$ и $d v / d t$ отличен от нуля. Тем самым, система уравнений (6.38) может быть переписана в виде (6.32), где

$$
\begin{aligned}
& X=\left(\frac{e^{22} g_{1}-e^{12} g_{2}}{D},-\frac{e^{22} f_{1}^{\mathbf{V}}-e^{12} f_{2}^{\mathbf{V}}}{D}\right), \\
& Y=\left(\frac{e^{11} g_{2}-e^{21} g_{1}}{D},-\frac{e^{11} f_{2}^{\mathbf{V}}-e^{21} f_{1}^{\mathbf{V}}}{D}\right),
\end{aligned}
$$


$\mathrm{a} f_{1}^{\mathbf{V}}=f_{4}^{1 \rho}, f_{2}^{\mathbf{V}}=f_{4}^{2 \rho}, g_{1}=h_{1}-\sum_{k=1}^{3} f_{k}^{1 \rho} \dot{\sigma}_{k}, g_{2}=h_{2}-\sum_{k=1}^{3} f_{k}^{2 \rho} \dot{\sigma}_{k}$.

Некоторые открытые вопросы. 1. В формулах для функций $A$ и $B$, приведенных в [96], фигурирует решение сингулярного интегрального уравнения

$$
\text { v.p. } \int_{\Gamma} \frac{\varphi(\xi) M(u, \xi)}{u-\xi} d \xi=q(u), \quad u \in \Gamma,
$$

где $\Gamma$ - отрезок прямой, а $(u, \xi) \mapsto M(u, \xi)$ - функция, голоморфная по $(u, \xi) \in$ $\Gamma \times \Gamma$, а именно: $\Gamma=\left[\sigma_{2}, \sigma_{3}\right], M(u, \xi)=\frac{1}{\omega}(u-\xi) \operatorname{ctg}\left(\frac{\pi}{\omega}(u-\xi)\right)+\frac{1}{\pi}$. Согласно теореме 6.1, интегральное уравнение (6.41) разрешимо в классе гёльдеровых функций, а его решение $\varphi$ единственно при определенном значении $\int \varphi$. Весьма желательно получить формулу для решения уравнения (6.41). Это позволило бы выписать абсолютно явный вид решения исходной задачи. Отметим, что в тех случаях, когда $M=$ const или же когда $\Gamma$ - замкнутая кривая (а не отрезок), явные формулы для решения уравнения (6.41) известны (см., например, [98]).

2. В [94] приведена аналогичная конструкция редукции к стандартной задаче оптимального управления оптимизационной задачи при нестационарном плоском потенциальном кавитационном обтекании идеальной жидкостью в случае, когда параметром управления является форма препятствия (см. рис. 13).

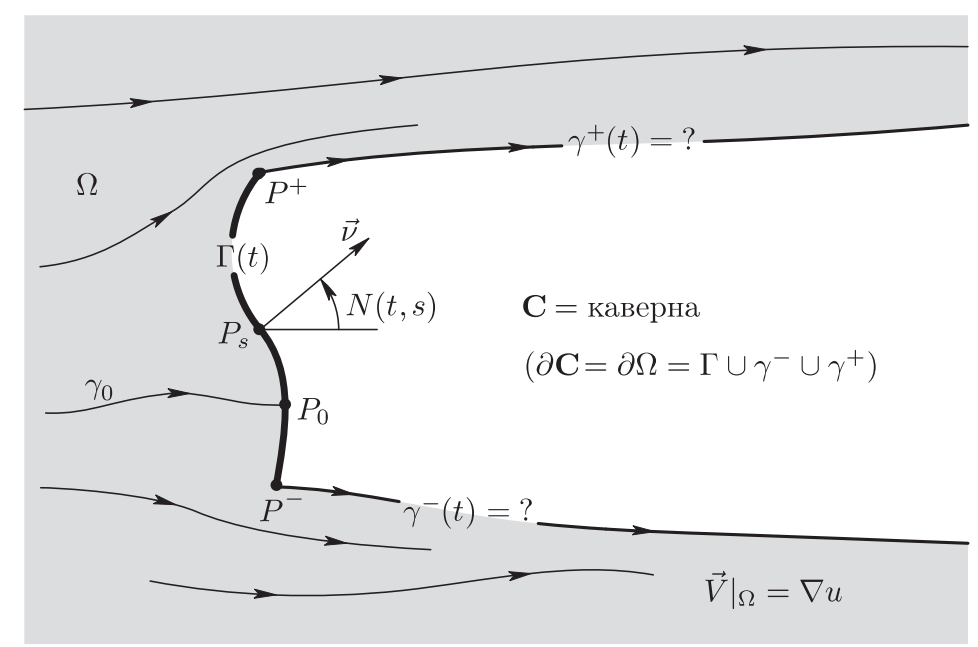

Рис. 13

Сложность этой задачи в том, что линии тока не совпадают с траекториями частиц жидкости, попадающих в некоторую искомую точку $P_{0}(t)$ разветвления потока на препятствии $\Gamma(t)$. Ведь они скользят к концевым точкам $P^{ \pm}(t)$ вдоль препятствия, меняющего свою форму. $\mathrm{K}$ тому же, образуемые ими после срыва с препятствия искомые кривые $\gamma^{ \pm}$также изменяются во времени. Было бы 
очень интересно обобщить представленные здесь и в [94] конструкции в двух направлениях: 1) нестационарное обтекание по схеме Фёппля-Лаврентьева в случае управления формой препятствия и 2) обтекание по той или иной схеме в ситуации, когда параметром управления является модуль скорости на обтекаемом препятствии. В частности, такого рода управлением можно пытаться воплотить идею Б. А. Луговцова (см. [93; § 37]) о снижении лобового сопротивления посредством частичной реализации условия прилипания при потенциальном обтекании вязкой жидкостью.

3. Полученные здесь и в [94] системы обыкновенных дифференциальных уравнений дают принципиальную возможность сведения исходной задачи оптимизации, относящейся к нестационарной нелинейной системе уравнений с частными производными в области со свободной границей, к стандартной задаче оптимального управления, связанной с системой обыкновенных дифференциальных уравнений. Конечно, эти системы уравнений достаточно сложны даже для численного их исследования. Но эта сложность отражает природу исходной задачи со свободной границей для уравнения движения Эйлера и уравнения неразрывности. Было бы наивно ожидать, что еще не предстоит серьезная вычислительная работа. Она ожидает специалистов вычислительной математики.

\section{§ 7. Стационарное обтекание по схеме Кирхгофа криволинейного препятствия, частично абсорбирующего энергию потока, и оценка максимально возможного КПД турбины в открытом потоке}

7.1. Как было отмечено во введении, эффективность турбины в открытом потоке (рек, океанских приливов и течений) зависит от того, насколько удалось конструктору найти “золотую середину" между двумя противоположными факторами: с одной стороны, не создавать потоку чрезмерное сопротивление (чтобы как можно большая его часть проходила через турбину), а с другой создать на его пути через турбину сопротивление ротору турбины, преодолевая которое он бы максимально отдавал свою энергию на его вращение.

Следуя [16], [17], будем моделировать турбину как препятствие, частично абсорбирующее установившийся потенциальный поток несжимаемой жидкости. Будем предполагать, что течение происходит по так называемой схеме Кирхгофа (см. рис. 14), когда за препятствием образуется бесконечная каверна с искомыми границами $\gamma_{0}$ и $\gamma_{1}$. Они являются линиями тока, сорвавшимися с кромок препятствия. Свобода выбора кривых $\gamma_{0}$ и $\gamma_{1}$ ограничена равенством давления на них постоянному давлению в каверне. Пусть $\Omega$ - область течения, т. е. область, внешняя относительно каверны. Обозначим через

$$
\vec{V}=\left(V_{x}, V_{y}\right): \Omega \ni(x, y) \mapsto \vec{V}(x, y)=\nabla u(x, y)
$$

скорость потока. Будем считать, что

$$
\vec{V}(x, y) \rightarrow\left(V_{\infty}, 0\right)=(1,0) \quad \text { при } \Omega \ni z=x+i y \rightarrow \infty .
$$

Геометрию препятствия представим в виде самонепересекающейся незамкнутой кривой $\Gamma$, длина которой (в выбранной системе единиц измерения) равна единице. Обозначим одну из крайних точек кривой через $P_{0}$, а через $P_{s}$, где $s \in[0,1]$, ту точку на $\Gamma$, расстояние до которой, измеренное вдоль $Г$ от $P_{0}$, 


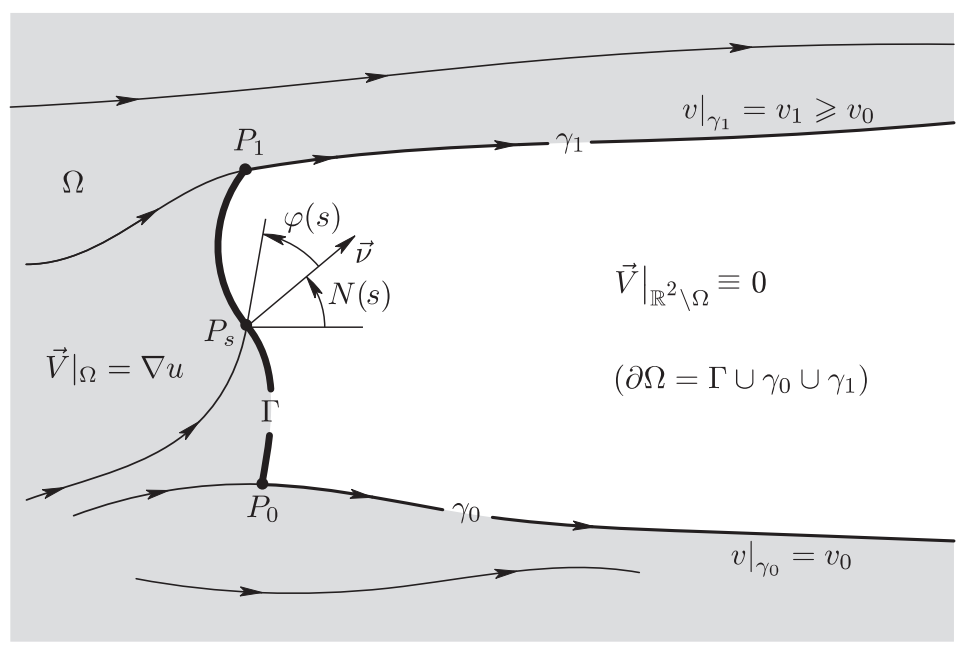

Рис. 14. Область течения $\Omega=\Omega_{\Gamma}$ и препятствие $\Gamma$, частично абсорбирующее набегающий поток, направленный в точке $P_{s} \in \Gamma$ под углом $\varphi(s) \in[-\pi / 2, \pi / 2]$ к нормали $\vec{\nu}(s)$ к $\Gamma ; N(s)$ - угол между осью $x$ и нормалью $\vec{\nu}(s)$

равно $s$. Будем считать, что ординаты $y_{0}$ и $y_{1}$ крайних точек $P_{0}$ и $P_{1}$ кривой $\Gamma$ подчинены условию

$$
D(\Gamma) \stackrel{\text { def }}{=} y_{1}-y_{0}>0 .
$$

Кривая Г полностью определяется функцией $N:[0,1] \ni s \mapsto N(s) \in \mathbb{R}$, задающей угол $N(s)$ между осью $x$ и нормалью $\vec{\nu}(s)$ к $\Gamma$ в точке $P_{s} \in \Gamma$. Будем предполагать, что

$$
|N(s)| \leqslant \pi / 2 \quad \forall s \in[0,1]
$$

и что срыв линий тока $\gamma_{0}$ и $\gamma_{1}$, охватывающих каверну, происходит с крайних точек $P_{0}$ и $P_{1}$ кривой $Г$.

Мощность турбины не превосходит той мощности, которую абсорбирует препятствие. Последняя (при тех или иных предположениях) может быть выражена интегралом вида

$$
\int_{\Gamma} f\left(s, V_{\vec{\nu}}(s), V_{\tau}(s)\right) d \Gamma, \quad f \in C\left(\Gamma \times \mathbb{R}^{2}\right) .
$$

Здесь $V_{\vec{\nu}}=\vec{V} \cdot \vec{\nu}$ (соответственно $\left.V_{\tau}=\vec{V} \cdot \vec{\tau}\right)$ - это скалярное произведение векторов $\vec{V}$ и $\vec{\nu}=(\cos N, \sin N)($ соответственно $\vec{V}$ и $\vec{\tau}=(-\sin N, \cos N))$.

Как максимизировать эту мощность? Будем считать, что для этого (кроме возможности выбора кривой $\Gamma$, заданной функцией $N$ ) можно в каждой точке $P_{s} \in \Gamma$ варьировать (соответствующим поворотом створок) то или иное направление вектора скорости $\vec{V}(s)$ абсорбируемого потока, а именно угол $\varphi(s) \in$ $[-\pi / 2, \pi / 2]$ между нормалью $\vec{\nu}(s)$ к $\Gamma$ (направленной в сторону каверны) и вектором $\vec{V}(s)$.

Ответы на вопросы о максимизации абсорбируемой мощности могут служить ориентиром при разработке "наилучшей” конструкции турбины. Такова 
мотивация постановки как задачи

$$
\mathfrak{F}_{1}(\Gamma, \varphi) \stackrel{\text { def }}{=} \int_{\Gamma} f\left(s, V_{\vec{\nu}}(s), V_{\tau}(s)\right) d \Gamma \rightarrow \sup ,
$$

так и задач следующего типа:

$$
\mathfrak{F}_{2}(\Gamma, \varphi) \stackrel{\text { def }}{=} \frac{\mathfrak{F}_{1}(\Gamma, \varphi)}{D(\Gamma)} \rightarrow \sup , \quad \mathfrak{F}_{3}(\Gamma, \varphi) \stackrel{\text { def }}{=} \frac{\mathfrak{F}_{1}(\Gamma, \varphi)}{H(\Gamma)} \rightarrow \sup ,
$$

где $D(\Gamma)$ уже было определено в (7.1), а

$$
H(\gamma)=\sup _{P_{s}=\left(x_{s}, y_{s}\right) \in \Gamma}|x(s)-x(0)| .
$$

Отметим, что число $D(\Gamma) / 2$ есть мощность (не возмущенного препятствием) равномерного потока $V_{0}=(1,0)$, протекающего в пределах сечения, совпадающего с проекцией кривой Г на ось $O y$. Подчеркнем также, что $\left|\vec{V}\left(P_{s}\right)\right|$ - искомая величина как в задаче (7.2), так и в задачах (7.3).

Задачи (7.2), (7.3) сформулированы в терминах функции $\vec{V}$, определенной в области $\Omega$ со свободной границей $\gamma_{0} \cup \gamma_{1}$, не являющейся непосредственно варьируемым аргументом функционалов $\mathfrak{F}_{k}(k=1,2,3)$. В следующем пункте эти задачи переформулированы в терминах функции Гельмгольца-Кирхгофа, что позволяет преодолеть трудности, связанные с наличием свободной границы области $\Omega$. Там же устанавливаются теоремы о разрешимости и приводятся формулы, представляющие решения задач.

7.2. В односвязной области $\Omega$ введем функцию $v: \Omega \rightarrow \mathbb{R}$, гармонически-сопряженную к функции $u$. Зафиксировав равенством $w\left(P_{0}\right)=0$ аддитивную константу аналитического отображения

$$
w: \Omega \ni z=x+i y \mapsto u(x, y)+i v(x, y) \in \mathbb{C},
$$

найдем (см. рис. 15) образ $Q=w(\Omega)$ области $\Omega$ и образ $\Lambda \cup \lambda_{0} \cup \lambda_{1}=w(\partial \Omega)$ ее границы $\partial \Omega=\Gamma \cup \gamma_{0} \cup \gamma_{1}$.

ЛЕмма 7.1. Кривая $\Lambda=w(\Gamma)$ задается формулой

$$
\Lambda=\left\{w\left(P_{s}\right)=u\left(P_{s}\right)+i v\left(P_{s}\right),[0,1] \ni s \mapsto P_{s} \in \Gamma\right\},
$$

¿de

$$
u\left(P_{s}\right)=\int_{0}^{s} V(r) \sin \varphi(r) d r, \quad v\left(P_{s}\right)=\int_{0}^{s} V(r) \cos \varphi(r) d r
$$

$\left(v\left(P_{s}\right)-\right.$ неубывающая функция $\left.s \in[0,1]\right)$, a $V(s)=\left|\vec{V}\left(P_{s}\right)\right|$.

ДокАЗАТЕЛЬство. Пусть $d s$ - дифференциал дуги кривой Г. Тогда (см. рис. 14)

$$
d x=-\sin N d s, \quad d y=\cos N d s .
$$

С другой стороны, $\vec{V}=\left(u_{x}, u_{y}\right)$ и потому $v_{x}=-u_{y}=-|\vec{V}| \sin \beta, v_{y}=u_{x}=$ $|\vec{V}| \cos \beta$, где $\beta=N+\varphi$ угол между осью $x$ и вектором $\vec{V}$. Следовательно,

$$
d u=u_{x} d x+u_{y} d y=|\vec{V}| \sin \varphi d s, \quad d v=v_{x} d x+v_{y} d y=|\vec{V}| \cos \varphi d s,
$$




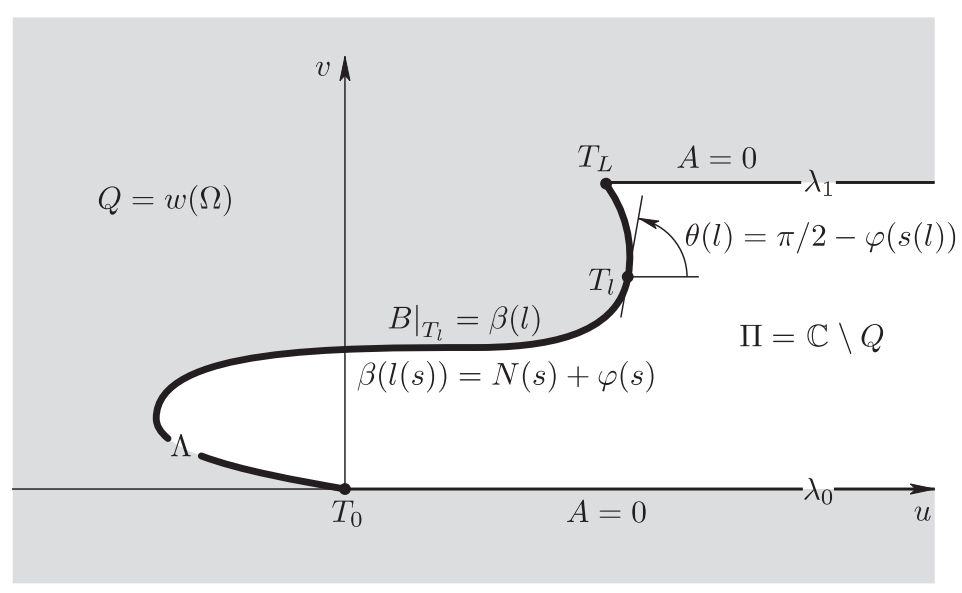

Рис. 15. Граничные условия функции $A+i B \stackrel{\text { def }}{=} \ln (d z / d w): Q=$ $w(\Omega) \rightarrow \mathbb{C}$ на $\partial Q=\Lambda \cup \lambda_{0} \cup \lambda_{1} \subset \Pi=\mathbb{C} \backslash Q$

что и доказывает лемму.

Очевидно, что в $w$-плоскости линии тока $\gamma_{0}$ и $\gamma_{1}$ представляются лучами

$$
\lambda_{0}=\left\{u \geqslant u\left(P_{0}\right), v=v\left(P_{0}\right)\right\} \quad \text { и } \quad \lambda_{1}=\left\{u \geqslant u\left(P_{1}\right), v=v\left(P_{1}\right)\right\} .
$$

Обозначим через $T_{l}$ ту точку на кривой $\Lambda$, расстояние до которой, измеренное вдоль $\Lambda$ от ее крайней точки $w\left(P_{0}\right)=0$, равно $l$. Заметим, что $T_{l(s)}=w\left(P_{s}\right)$, где $s$ - натуральный параметр точки $P_{s} \in \Gamma$, а

$$
l(s) \stackrel{(7.4),(7.5)}{=} \int_{0}^{s} V(r) d r, \quad s \in[0,1],
$$

где априори неизвестная функция $s \mapsto V(s)=\left|\vec{V}\left(P_{s}\right)\right|$ является искомой как в задаче (7.2), так и в задачах (7.3). В случае задачи (7.2) это относится и к длине $L=l(1)$ кривой $\Lambda$. Отметим, что $L \leqslant 1$, поскольку $V \leqslant 1$.

Не уточняя здесь пока функциональные классы, к которым принадлежат аргументы функционалов $\mathfrak{F}_{k}$, будем считать, что они таковы, что функция $l: s \stackrel{(7.6)}{\longmapsto} l(s)$ строго монотонна. Тогда, учитывая, что $l^{\prime}(s) \geqslant 0$ для любого $s \in[0,1]$, а $\vec{V}_{\infty}=(1,0)$, получим, согласно принципу сохранения границ [46], что отображение $w: z \mapsto w(z)$ однолистно отображает область $\Omega$ на область $Q=w(\Omega)$, дополнительную к (замкнутой в $\mathbb{C}$ ) “полу"-полосе П, лежащей между лучами $\lambda_{0}, \lambda_{1}$ и ограниченной слева кривой $\Lambda$.

Поскольку функция $l: s \mapsto l(s)$ строго монотонна, она имеет обратную, которая выражается формулой

$$
s:[0, L] \ni l \mapsto s(l)=\int_{0}^{l} e^{A\left(u_{l}, v_{l}\right)} d l .
$$

Здесь и ниже $\left(u_{l}, v_{l}\right)$ - координаты точки $T_{l} \in \Lambda$, а $A$ - это вещественная часть функции Гельмгольца-Кирхгофа

$$
A+i B: Q=w(\Omega) \ni w=u+i v \mapsto A(u, v)+i B(u, v) \stackrel{\text { def }}{=} \ln \frac{d z}{d w} \in \mathbb{C},
$$


определенной в односвязной области $Q$. Из формул (7.5) вытекает следующее утверждение.

ЛЕмма 7.2. Кривая $\Lambda$ полностъю определяется функиией

$$
\theta:[0, L] \ni l \mapsto \theta(l)=\frac{\pi}{2}-\varphi(s(l)) \in[0, \pi],
$$

задающей как длину $L=|\Lambda|$ этой кривой, так и угол $\theta(l)$ между осъю $O и$ и касательной $к$ ней в точке $T_{l} \in \Lambda$.

Найдем граничные значения функции Гельмгольца-Кирхгофа. Легко видеть, что

$$
A=0 \quad \text { на лучах } \lambda_{0}=w\left(\gamma_{0}\right) \text { и } \lambda_{1}=w\left(\gamma_{1}\right) .
$$

Действительно, положение линий тока $\gamma_{0}$ и $\gamma_{1}$ определяется равенством давления на них как со стороны потока, так и со стороны каверны. В каверне давление постоянно. Поэтому, в силу интеграла Бернулли (см., например, [99]), $|\nabla u|=V_{\infty}=1$ на $\gamma_{0}$ и $\gamma_{1}$. Остается вспомнить, что $A(u, v)=-\ln \left|w_{z}^{\prime}(z)\right|_{z=z(w)} \mid$. Столь же просто устанавливается формула

$$
B\left(u_{l}, v_{l}\right)=\beta(l), \quad \text { где }\left.\beta(l)\right|_{l=l(s)} \stackrel{\text { def }}{=} N(s)+\varphi(s) .
$$

В самом деле, $\cos \varphi d u \stackrel{(7.5)}{=} \sin \varphi d v$ и потому

$$
B=\arg d z-\arg d w=\left(\frac{\pi}{2}+N\right)-\left(\frac{\pi}{2}-\varphi\right) .
$$

Наконец, на бесконечности имеем

$$
A+i B \rightarrow A_{\infty}=0 \quad \text { при } Q \ni w \rightarrow \infty,
$$

поскольку $\vec{V} \rightarrow(1,0)$ при $\Omega \ni z \rightarrow \infty$.

ЗАмЕчАНИЕ 7.1. Удобно нормировать длину $L$ кривой $\Lambda$ с помощью замены $(\xi, \eta)=(u / L, v / L)$. Всюду в дальнейшем будем считать, что такая замена выполнена. Однако новые переменные $(\xi, \eta)$ будут по-прежнему обозначаться через $(u, v)$.

Напомним, что $N(s)$ есть угол между осью $x$ и нормалью к $\Gamma$ в точке $P_{s} \in \Gamma$; $\theta(\cdot)=\pi / 2-\varphi(s(\cdot))$, а $\beta(s)=N(s)+\varphi(s)$ - это то значение, которое принимает функция $B$ (гармонически-сопряженная к $A$ ) в точке $w\left(P_{s}\right)$. Анонсированная выше переформулировка задач (7.2), (7.3) в терминах функции Гельмгольца-Кирхгофа базируется на нижеследующих леммах.

ЛЕмма 7.3. $\mathfrak{F}_{1}(\Gamma, \varphi)=\mathfrak{G}_{1}(\theta, \beta)$, где (с учетом замечания 7.1)

$$
\mathfrak{G}_{1}(\theta, \beta)=\left.L \int_{0}^{1} f\left(s, e^{-A\left(u_{l}, v_{l}\right)} \cos \varphi(s), e^{-A\left(u_{l}, v_{l}\right)} \sin \varphi(s)\right)\right|_{s \stackrel{(7.7)}{=} s(l)} e^{A\left(u_{l}, v_{l}\right)} d l,
$$

$a\left(u_{l}, v_{l}\right)-$ координатъ точки $T_{l} \in \Lambda$.

Действительно, якобиан отображения $z \mapsto w(z)$ равен $e^{A}, \mathrm{a}$

$$
\vec{V}(x, y)=\exp (-A(u, v)+i B(x, y)), \quad \text { где } u+i v=w(x+i y) .
$$


Лемма 7.4. $D(\Gamma)=\mathfrak{D}(\theta, \beta)$, где (с учетом замечания 7.1)

$$
\mathfrak{D}(\theta, \beta)=\left.L \int_{0}^{1} e^{A\left(u_{l}, v_{l}\right)} \cos N(s)\right|_{s} ^{(7.7)} s(l) d l .
$$

Действительно, $d u=d l \cos \theta, d v=d l \sin \theta, \stackrel{(7.10)}{=} N+\varphi$. Поэтому

$$
\mathfrak{D}(\theta, \beta)=\left.\int_{\Lambda} e^{A}[\cos B d v+\sin B d u] \stackrel{(7.8)}{=} L \int_{0}^{1} e^{A\left(u_{l}, v_{l}\right)} \cos N(s)\right|_{s} \stackrel{(7.7)}{=} s(l) d l .
$$

Точно так же доказывается следующее утверждение.

ЛЕмма 7.5. $H(\Gamma)=\mathfrak{H}(\theta, \beta)$, где (с учетом замечания 7.1)

$$
\mathfrak{H}(\theta, \beta)=L \sup _{0 \leqslant l \leqslant 1}\left|\int_{0}^{l} e^{A\left(u_{l}, v_{l}\right)} \sin N(s)\right| \stackrel{(7.7)}{=} s(l) d l \mid .
$$

Хотя $\mathfrak{D}(\theta, \beta)$ и $\mathfrak{H}(\theta, \beta)$ представлены в формулах (7.13) и (7.14) через функцию $A$, зависящую от $\beta$ и $\theta$ (т. е. от кривой $\Lambda$, на которой функция $B: Q \rightarrow \mathbb{C}$, гармонически-сопряженная к $A$, равна $\beta)$, однако и $\mathfrak{D}(\theta, \beta)$, и $\mathfrak{H}(\theta, \beta)$ зависят лишь от кривой $\Gamma$ и выражают ее геометрические характеристики: "размер" по вертикали и горизонтальное "отклонение" от вертикали $x=0$.

Из (7.7)-(7.11) и лемм 7.3-7.5 вытекает следующая теорема.

ТЕОРема 7.1 [91]. Пусть функииональные классы, на которых рассматриваются функииональ

$$
\mathfrak{F}_{1}(\Gamma, \varphi), \quad \mathfrak{F}_{2}(\Gamma, \varphi), \quad \mathfrak{F}_{3}(\Gamma, \varphi)
$$

(заданные формулами (7.2), (7.3)), таковы, что отображение $w: \bar{\Omega} \rightarrow \bar{Q}$ гомеоморфно, а в $\Omega$ однолистно. Пусть $\left(\hat{\theta}_{k}, \hat{\beta}_{k}\right)$ - решение задачи

$$
\mathfrak{G}_{k}(\theta, \beta) \rightarrow \sup
$$

где функционал $\mathfrak{G}_{1}(\theta, \beta)$ определен формулой (7.12), а

$$
\mathfrak{G}_{2}(\theta, \beta) \stackrel{\text { def }}{=} \frac{\mathfrak{G}_{1}(\theta, \beta)}{\mathfrak{D}(\theta, \beta)}, \quad \mathfrak{G}_{3}(\theta, \beta) \stackrel{\text { def }}{=} \frac{\mathfrak{G}_{1}(\theta, \beta)}{\mathfrak{H}(\theta, \beta)} .
$$

Тогда задача

$$
\mathfrak{F}_{k}(\Gamma, \varphi) \rightarrow \sup
$$

имеет решение $\left(\widehat{\Gamma}_{k}, \hat{\varphi}_{k}\right)$ и пара функциц $\left(\widehat{N}_{k}, \hat{\varphi}_{k}\right)$, представляющая это решение, определяется следующим образом:

$$
\left.\widehat{N}_{k}(s)\right|_{s=s_{k}(l)}=B\left(s_{k}(l)\right),\left.\quad \hat{\varphi}_{k}(s)\right|_{s=s_{k}(l)}=\frac{\pi}{2}-\hat{\theta}(l) .
$$

Здесь функиия $s_{k}$ определена согласно формуле (7.7), а фигурирующая в этой формуле функиия Гельмгольца-Кирхгофа $A+i B: Q \rightarrow \mathbb{C}$ удовлетворлет условиям (7.9)-(7.11), где в качестве функиии $\beta$ (в формуле (7.10)) взята функицяя $\hat{\beta}_{k}$. 
7.3. Приведем простейший (но полезный с точки зрения численного анализа) пример, когда выполнены предположения теоремы 7.1, т. е. отображение $w: \bar{\Omega} \rightarrow \bar{Q}$ гомеоморфно, в $\Omega$ оно однолистно, а задачи (7.15) разрешимы. Определим с этой целью множество $\mathfrak{M}_{\rho}$ по заданному натуральному числу $M$ и положительному числу $\rho$. Множество $\mathfrak{M}_{\rho}$ - это класс кусочно постоянных функций

$$
\theta \in L^{\infty}((0, L) ; \mathbb{R}) \quad \text { и } \quad \beta \in L^{\infty}((0, L) ; \mathbb{R}),
$$

каждая из которых характеризуется тремя $M$-мерными векторами

$$
\vec{\sigma}=\left(\sigma_{1}, \ldots, \sigma_{M}\right), \quad \vec{\theta}=\left(\theta_{1}, \ldots, \theta_{M}\right) \quad \text { и } \quad \vec{\beta}=\left(\beta_{1}, \ldots, \beta_{M}\right)
$$

такими, что (для любого $j=1, \ldots, M$ )

$$
\sigma_{j} \geqslant \rho>0, \quad L \stackrel{\text { def }}{=} \sum_{j=1}^{M} \sigma_{j} \leqslant \frac{1}{\rho}, \quad \theta_{j}=\frac{\pi}{2}-\varphi_{j}, \quad \beta_{j}=N_{j}+\varphi_{j},
$$

где числа $\varphi_{j}, N_{j}(j=1, \ldots, M)$ удовлетворяют условиям

$$
\left|\varphi_{j}\right| \leqslant \frac{\pi}{2}, \quad\left|N_{j}\right| \leqslant \frac{\pi}{2},
$$

a

$$
\left|\beta_{j+1}-\beta_{j}\right| \leqslant \pi-\rho \quad \text { при } 1 \leqslant j \leqslant M-1 .
$$

Элемент $(\theta, \beta)$ множества $\mathfrak{M}_{\rho}$ определяется так:

$$
\theta(l)=\theta_{j}, \quad \beta(l)=\beta_{j} \quad \text { при } l_{j-1}<l<l_{j} \text { для } j=1, \ldots, M .
$$

Здесь

$$
l_{0}=0, \quad l_{1}=\sigma_{1}, \quad \ldots, \quad l_{j}=l_{j-1}+\sigma_{j}, \quad \ldots, \quad l_{M}=L .
$$

Пара $(\theta, \beta) \in \mathfrak{M}_{\rho}$ определяет в свою очередь

1 ) ломаную $\Lambda=\bigcup \Lambda_{j}$ (см. рис. 15$)$, составленную из отрезков $\Lambda_{j}, j$-й из которых имеет длину $\sigma_{j}$, наклонен к оси $O u$ под углом $\theta_{j} \in[0, \pi]$ и примыкает к отрезку $\Lambda_{j-1}$ (отрезок $\Lambda_{1}$ исходит из начала координат $\left.(u, v)=(0,0)\right)$;

2) значение функции $B$ на $\Lambda_{j}$, а именно: $\left.B\right|_{\Lambda_{j}}=\beta_{j}$.

Тем самым, пара $(\theta, \beta) \in \mathfrak{M}_{\rho}$ задает, согласно формулам (7.9)-(7.11)) функцию Гельмгольца-Кирхгофа $A+i B$.

Теорема 7.2 [91]. Задача (7.15) имеет решение на множестве $\mathfrak{M}_{\rho}$, отвечающее ей отображение $w: \bar{\Omega} \rightarrow \bar{Q}$ гомеоморфно и в $Q$ однолистно.

В самом деле согласно доказательству предложения 1.2, из (7.21), (7.22) следует включение $\left.e^{ \pm A}\right|_{\Lambda} \in L^{1}(\Lambda)$ и потому, в силу $\sigma_{j} \geqslant \rho>0$ (см. (7.20)), функционалы (7.12) и (7.16) непрерывны относительно компонент векторов (7.19).

Некоторые открытые вопросы. 1. Каковы численные характеристики решений задач (7.17) на классах ломаных кривых, определенных в п. 7.3?

2. Каково максимальное КПД “турбины” в более общих случаях, в том числе (cp. §6) в случае нестационарного обтекания с тем или иным граничным управлением, как при кавитационном, так и при вихревом следе? 


\section{Глава IV. Экспоненциально точные высокочастотные асимптотики}

\section{§ 8. Векторные поля, определяющие экспоненциально точные высокочастотные асимптотики гармонических функций}

В этом и следующем параграфах рассматривается построение асимптотики тех гармонических функций, заданных в плоской области, которые быстро осциллируют на границе этой области. Такого рода задачи изучались в разных работах, в том числе и в заметке [56], написание которой было инициировано вопросом, на который внимание автора обратила О. А. Олейник. Ниже дается развернутое изложение той части этой заметки, которая имеет отношение к функционально-геометрическому методу. Исходная идея весьма проста. Она опирается на то, что все сводится к построению асимптотики функции, экспоненциально быстро стабилизирующейся при отходе от границы вглубь области. Поэтому если для заданной осцилляции граничного следа этой гармонической функции асимптотика известна в том случае, когда область $\Omega$ есть диск $D$, то асимптотика в любой другой, скажем, односвязной области $\Omega$ может быть построена с помощью такого конформного преобразования приграничных окрестностей диска и этой области $\Omega$, которое изометрично трансформирует границы диска $D$ и области $\Omega$ (будто бы эти границы $\partial D$ и $\partial \Omega$ сделаны из нерастяжимой гибкой проволочки).

Очевидно, что необходимым условием существования такого отображения является равенство длин границ $\partial D$ и $\partial \Omega$, а также аналитичность кривой $\Gamma=\partial \Omega$. Оказывается, что при выполнении этих необходимых условий рассматриваемый метод позволяет выписать сравнительно простые явные формулы для такого рода отображений.

8.1. Для малых значений параметра $\varepsilon=1 / M(M \in \mathbb{N})$ будем здесь строить такое асимптотическое приближение $u_{\varepsilon}$ решения $U_{\varepsilon}$ краевой задачи

$$
\left.\Delta U_{\varepsilon}\right|_{\Omega}=0,\left.\quad\left(U_{\varepsilon}+\lambda \frac{\partial U_{\varepsilon}}{\partial \nu}\right)\right|_{s \in \Gamma=\partial \Omega}=f_{\varepsilon}(s),
$$

которое при $f_{\varepsilon}(s)=f(s / \varepsilon)$, где ${ }^{6} f \in H^{\beta}(\Gamma)$, удовлетворяет следующей оценке:

$$
\left\|u_{\varepsilon}-U_{\varepsilon}\right\|_{H^{\alpha}(\Omega)} \leqslant C_{\alpha} e^{-\tau / \varepsilon}, \quad \text { где } \tau>0, \alpha=\beta+\frac{1}{2}+\operatorname{sign} \lambda \geqslant 0 .
$$

Здесь $\lambda=$ const $\geqslant 0, \partial / \partial \nu$ - дифференцирование по внешней нормали к $\Gamma, H^{\alpha}-$ пространство Соболева с показателем гладкости $\alpha$. Граница $Г$ области $\Omega \Subset \mathbb{R}^{2}$ (для простоты односвязная) предполагается аналитической. Ее длина $|\Gamma|$ равна $2 \pi$. Точка $s \in \Gamma$ отождествляется с натуральным параметром границы. Изометрично отображая $Г$ на единичную окружность $\mathbb{T}=\left\{e^{i \sigma}, \sigma \in R / 2 \pi \mathbb{Z}\right\}$, можно считать, что $f \in H^{\beta}(\mathbb{T})$.

\footnotetext{
${ }^{6}$ При $\beta<-1 / 2$ (что допускается в случае $\lambda>0$ ) функция $f$ необходимо является обобщенной. Это не противоречит физике дела, поскольку на границе $\Gamma$ измеряют вовсе не поточечные значения нормальной производной $\partial u / \partial \nu$, а поток через границу в пределах локализации, определяемой измерительным прибором. Иначе говоря, речь идет о задании мер на границе Г. Первая работа [100], математически точно выражающая это обстоятельство, послужила (по словам Э. Мадженеса) исходным импульсом для трехтомных исследований Ж.-Л. Лионса и Э. Мадженеса по неоднородным граничным задачам [101].
} 
Без ограничения общности можно считать, что функция $f$ (вообще говоря, обобщенная) имеет нулевое среднее: $\int_{\mathbb{T}} f(s) d s=0$. Эта функция определяет быстро осциллирующую около нулевого значения граничную функцию

$$
f_{\varepsilon}: \mathbb{T} \ni e^{i \sigma} \mapsto f(M \sigma)=\sum_{k \geqslant 1}\left(a_{k} \cos M k \sigma+b_{k} \sin M k \sigma\right),
$$

при этом

$$
\|f\|_{\beta}^{2} \stackrel{\text { def }}{=} \sum_{k \geqslant 1} k^{2 \beta}\left(\left|a_{k}\right|^{2}+\left|b_{k}\right|^{2}\right)<\infty .
$$

Если $\Omega$ есть диск $D$, а $(\rho, \sigma)$ - его полярные координаты, то функция

$$
v_{\varepsilon}:(\rho, \sigma) \mapsto \sum_{k \geqslant 1} \frac{\rho^{M k}}{1+M k \lambda}\left(a_{k} \cos M k \sigma+b_{k} \sin M k \sigma\right)
$$

является, очевидно, точным решением задачи (8.1) и

$$
\left\|v_{\varepsilon}(\rho, \cdot)\right\|_{\beta} \stackrel{(8.4)}{\leqslant} \rho^{1 / \varepsilon}\|f\|_{\beta} .
$$

Это решение принадлежит (см., например, [102]) пространству Соболева $H^{\alpha}(\Omega)$ (где $\alpha=\beta+1 / 2+\operatorname{sign} \lambda \geqslant 0)$, и оно единственно в этом классе, но не единственно в классе $H^{-\mu}(\Omega)$ при любом $\mu>0$ (например, потому, что ядро Пуассона задачи Дирихле принадлежит $H^{-\mu}(\Omega)$ при $\left.\mu>0\right)$.

Имея решение в диске $D$, трансформируем его в асимптотическое решение исходной задачи (8.1). Для этого построим конформное изометрическое отображение аналитической кривой $\Gamma$ на единичную окружность $\mathbb{T}=\{\zeta \in \mathbb{C} \mid$ $|\zeta|=1\}$. Иными словами, речь идет о таком однолистном отображении $\zeta$ : $V_{\Gamma} \ni z \mapsto \zeta(z) \in V_{\mathbb{T}}$ некоторой окрестности $V_{\Gamma}$ кривой $Г$ на соответствующую окрестность $V_{\mathbb{T}}$ окружности $\mathbb{T}$, которое изометрично отображает окружность на кривую Г, т. е. удовлетворяет следующему условию:

$$
\left|z^{\prime}(\zeta)\right| \equiv 1 \quad \text { для } \zeta \in \mathbb{T} \text {. }
$$

Такое отображение, как уже было отмечено в 2 , может быть задано формулой

$$
\zeta: V_{\Gamma} \ni z \mapsto \zeta(z)=1+\int_{z_{0}}^{z} e^{p+i q} d z, \quad z_{0} \in \Gamma,
$$

в которой функция Гельмгольца-Кирхгофа $p+i q: V_{\Gamma} \rightarrow \mathbb{C}$ (обозначенная на этот раз иными буквами, чем раньше) характеризуется условиями

$$
\left.q\right|_{s \in \Gamma}=s-N(s) \quad \text { и }\left.\quad p\right|_{s \in \Gamma}=0 \quad\left(\left.\Rightarrow \frac{\partial q}{\partial \nu}\right|_{\Gamma}=0\right),
$$

где $N(s)$ - угол между осью и внешней нормалью к $\Gamma$ в точке $s$. Равенство нулю нормальной производной функции $q$ влечет постоянство функции $p$ на $\Gamma$. Равенство нулю этой постоянной эквивалентно условию изометрии (8.7).

Заметим, что гармоническая функция $v_{\varepsilon} \circ \zeta$, заданная формулой

$$
\left.\left(v_{\varepsilon} \circ \zeta\right)\right|_{z=x+i y}=v_{\varepsilon}(\rho(x, y), \sigma(x, y)), \quad \zeta(x+i y)=\rho(x, y) e^{i \sigma(x, y)},
$$

удовлетворяет, в силу свойства изометрии (8.7), граничному условию задачи (8.1). 
Возьмем две кольцевые области $V_{\mathbb{T}}^{0}$ и $V_{\mathbb{T}}^{1} \subset V_{\mathbb{T}}^{0} \subset V_{\mathbb{T}}$, определяемые радиусами $\rho_{k}$ малой окружности кольца $V_{\mathbb{T}}^{k}=\left\{\rho_{k}<\rho<1\right\}$, и рассмотрим их прообразы $V_{\Gamma}^{k}=z\left(V_{\mathbb{T}}^{k}\right) \subset V_{\Gamma}$ при отображении $\zeta: V_{\Gamma} \rightarrow V_{\mathbb{T}}$. Пусть $\chi \in C^{\infty}(\Omega)-$ так называемая срезающая функция, такая, что: $\chi=1$ в $V_{\Gamma}^{1}$ и $\chi=0$ вне $V_{\Gamma}^{0}$.

Определим функцию $u_{\varepsilon}$ формулой $u_{\varepsilon}=\chi v_{\varepsilon} \circ \zeta$, полагая

$$
\begin{array}{ll}
u_{\varepsilon}(x, y)=\chi(x, y) v_{\varepsilon}(\rho(x, y), \sigma(x, y)) & \text { в }(x, y) \in V_{\Gamma}^{0} \\
u_{\varepsilon}(x, y)=0 & \text { вне } V_{\Gamma}^{0} .
\end{array}
$$

Положим $H_{+}^{t}(Q)=H^{t_{+}}(Q)$, где $t_{+} \stackrel{\text { def }}{=} \max \{t, 0\}$. Пусть $W=V_{\Gamma}^{0} \backslash V_{\Gamma}^{1}$. Имеем

$$
\left\|\Delta u_{\varepsilon}\right\|_{H_{+}^{\alpha-2}\left(V_{\Gamma} \backslash W\right)}=0, \quad\left\|\Delta u_{\varepsilon}\right\|_{H_{+}^{\alpha-2}(W)} \stackrel{(8.6)}{\leqslant} C_{\alpha} \rho_{1}^{1 / \varepsilon} .
$$

Тем самым, функция $R_{\varepsilon}=u_{\varepsilon}-U_{\varepsilon}$ удовлетворяет условиям

$$
\left\|\Delta R_{\varepsilon}\right\|_{H_{+}^{\alpha-2}(\Omega)} \leqslant C_{\alpha} \rho_{1}^{1 / \varepsilon},\left.\quad\left(R_{\varepsilon}+\lambda \frac{\partial R_{\varepsilon}}{\partial \nu}\right)\right|_{\Gamma}=0 .
$$

Отсюда, согласно эллиптической априорной оценке (см., например, [101], [102]), получаем анонсированное неравенство (8.2) с $\tau=\ln \left(1 / \rho_{1}\right)>0$.

ЗАмЕчаниЕ 8.1. Оценка (8.2) остается в силе и в более общем случае, когда функция $f_{\varepsilon}$ определяется не формулой (8.3), а подчинена условию, при котором, как и в $(8.12),\left\|\Delta u_{\varepsilon}\right\|_{H_{+}^{\alpha-2}(W)} \leqslant C_{\alpha} \rho_{1}^{1 / \varepsilon}$. А если $\left\|\Delta u_{\varepsilon}\right\|_{H_{+}^{\alpha-2}(W)} \leqslant C_{\alpha} \varepsilon^{\omega}$, то вместо (8.2) будем иметь $\left\|u_{\varepsilon}-U_{\varepsilon}\right\|_{H^{\alpha}(\Omega)} \leqslant C_{\alpha} \varepsilon^{\omega}$.

ЗАмЕчАНИЕ 8.2. Ввиду (8.8) и (8.10), в формуле (8.11), задающей искомую асимптотику $u_{\varepsilon}$, неявно фигурируют гармонически-сопряженные функции $p$ и $q$, определенные в некоторой окрестности границы Г и удовлетворяющие, согласно (8.9), следующим данным Коши:

$$
\left.p\right|_{s \in \Gamma}=0,\left.\quad \frac{\partial p}{\partial \nu}\right|_{s \in \Gamma}=G(s) \quad \text { и }\left.\quad q\right|_{s \in \Gamma}=F(s),\left.\quad \frac{\partial q}{\partial \nu}\right|_{s \in \Gamma}=0 .
$$

Здесь $F(s)=s-N(s), G(s)=-F^{\prime}(s)$. Формулы для решения этой задачи даны в теореме 2.1. Тем не менее, в некоторых случаях можно получить более явное (и геометрически наглядное!) асимптотическое приближение с помощью конструкции, изложенной в следующем пункте.

8.2. Не ограничивая общность, мы предположили, что функция $f$, заданная на окружности $\mathbb{T}$ имеет нулевое среднее: $\int_{\mathbb{T}} f(s) d s=0$. Рассмотрим случай задачи Дирихле и предположим дополнительно, что функция $f$ нечетна относительно некоторой точки $t_{*} \in \mathbb{T}$. Даже если функция $f$ разрывна в $t_{*}$, будем считать эту точку ее нулем, поскольку в эту точку упирается нулевая линия уровня функции $u_{\varepsilon}=v_{\varepsilon} \circ \zeta$. В силу нечетности, функция $f$ обращается в нуль в двух диаметрально противоположных точках: в $t_{*}$ и в $t_{*}+\pi$. Эти нули трансформируются в $2 M$ равномерно распределенных вдоль $Г$ нулей граничной функции $f_{\varepsilon}: s \mapsto f(s / \varepsilon)$, где $1 / \varepsilon=M \in \mathbb{N}$. 
Зададимся таким вопросом: вдоль каких кривых распространяются внутрь области эти $2 M$ граничных нулей функции $f_{\varepsilon}$ ? Точнее, как, не выписывая заранее точную асимптотику $u_{\varepsilon}$ решения $U_{\varepsilon}$, найти ее нулевые линии уровня, проходящие через эти граничные нули?

Ответ на этот вопрос позволяет мгновенно получить не только содержательную информацию о графике функции $u_{\varepsilon}$, но и найти саму асимптотику $u_{\varepsilon}$ решения $U_{\varepsilon}$. Ответ на поставленный вопрос дает следующая легко доказываемая теорема.

ТеОрема 8.1. Пусть функиия $f: \mathbb{T} \rightarrow \mathbb{R}$ нечетна. Тогда искомые нулевые линии уровня функции $u_{\varepsilon}=v_{\varepsilon} \circ \zeta$, проходящие через $2 M$ равномерно распределенных нулей граничной функции $f_{\varepsilon}: s \mapsto f(s / \varepsilon)$, вкладываются в векторное поле $\pi_{\Gamma}$ трансверсалей $\kappa \Gamma$, которые являются образом радиусов окружности при отображении $\zeta^{-1}: V_{\mathbb{T}} \rightarrow V_{\Gamma}$. Это отображение $z=\zeta^{-1}$, обратное $\kappa \zeta: V_{\Gamma} \rightarrow V_{\mathbb{T}}$, задается формулой

$$
z: V_{\mathbb{T}} \ni \zeta=\rho e^{i \sigma} \mapsto z(\zeta)=z_{0}+\int_{1}^{\zeta} \exp \{A+i B\} d \zeta \in V_{\Gamma}, \quad z_{0} \in \Gamma,
$$

в которой функиия Гелвмгольца-Кирхгофа $A+i B$ определяется по решению следующей задачи Коши:

$$
\left.B(\rho, \sigma)\right|_{\rho=1}=N(\sigma)-\sigma,\left.\quad B_{\rho}\right|_{\rho=1} \stackrel{\left.A\right|_{\rho=1}=0}{=} 0, \quad \rho=|\zeta|, \quad \sigma=\arg \zeta .
$$

A именно,

$$
\begin{aligned}
& A: V_{S} \ni \zeta=\rho e^{i \sigma} \mapsto A(\rho, \sigma)=\frac{1}{2} \sum_{k \geqslant 1}\left(\rho^{k}-\rho^{-k}\right)\left[G_{k} \cos k \sigma-F_{k} \sin k \sigma\right], \\
& B: V_{S} \ni \zeta=\rho e^{i \sigma} \mapsto B(\rho, \sigma)=F_{0}+\frac{1}{2} \sum_{k \geqslant 1}\left(\rho^{k}+\rho^{-k}\right)\left[F_{k} \cos k \sigma+G_{k} \sin k \sigma\right],
\end{aligned}
$$

где $F_{k}$ и $G_{k}$ - это коэффициенты, фигурирующие в следующем представлении:

$$
\mathbb{T} \ni \sigma \mapsto B(1, \sigma)=F_{0}+\sum_{k \geqslant 1}\left(F_{k} \cos k \sigma+G_{k} \sin k \sigma\right) .
$$

Действительно, требуемая изометрия отображения (8.14) на окружности эквивалентна тождеству $\left.e^{A}\right|_{\rho=1} \stackrel{\text { def }}{=} d s(\sigma) / d \sigma \equiv 1$, где $s(\sigma)$ - длина дуги кривой $\Gamma$, параметризованная точкой $e^{i \sigma} \in \mathbb{T}$. Без ограничения общности можно считать, что $s(\sigma)=\sigma$. Отсюда $N(s(\sigma))=N(\sigma)$ и потому $\left.B\right|_{\rho=1}=N(\sigma)-\sigma$.

8.3. Представленные выше построения можно в какой-то мере распространить на эллиптические уравнения вида

$$
\mathfrak{A}(u) \stackrel{\text { def }}{=} a_{11}(x, y) u_{x x}+2 a_{12}(x, y) u_{x y}+a_{22}(x, y) u_{y y}+F\left(x, y, u, u_{x}, u_{y}\right)=0,
$$

где функции $a_{i j}:(x, y) \mapsto a_{i j}(x, y)$ и $F$ аналитичны. Допустим, речь идет о задаче (8.1), в которой оператор Лапласа заменен на оператор $\mathfrak{A}$ и рассматривается, скажем, задача Дирихле. В этом случае, по существу, все сводится к построению в окрестности границы Г двух функций

$$
(x, y) \mapsto \rho(x, y) \quad \text { и } \quad(x, y) \mapsto \varphi(x, y),
$$


задающих полярные координаты в окрестности единичной окружности и удовлетворяющих следующим условиям. Во-первых,

$$
\rho_{x}=\beta \varphi_{x}+\gamma \varphi_{y}, \quad-\rho_{y}=\alpha \varphi_{x}+\beta \varphi_{y}
$$

где

$$
\alpha=\frac{a_{11}}{\sqrt{a_{11} a_{22}-a_{12}^{2}}}, \quad \beta=\frac{a_{12}}{\sqrt{a_{11} a_{22}-a_{12}^{2}}}, \quad \gamma=\frac{a_{22}}{\sqrt{a_{11} a_{22}-a_{12}^{2}}} .
$$

Во-вторых,

$$
\left.\rho\right|_{(x, y) \in \Gamma}=1,\left.\quad \frac{\partial \varphi}{\partial \tau}\right|_{(x, y) \in \Gamma}=1,
$$

где $\partial / \partial \tau$ - дифференцирование вдоль $Г$.

Как известно (см., например, [103; гл. IV, §8]), при замене переменных (8.19)-(8.20) уравнение (8.22) примет в окрестности границы Г такой вид:

$$
\rho^{2} v_{\rho \rho}+\rho v_{\rho}+v_{\varphi \varphi}+G\left(\rho, \varphi, v, v_{\rho}, v_{\varphi}\right)=0
$$

где $v(\rho(x, y), \varphi(x, y))=u(x, y)$. Данные Коши (8.21) для эллиптической системы (8.19)-(8.20) обеспечивают изометрическое преобразование границы Г в окружность $\mathbb{T}$ радиуса $\rho=1$. Поэтому исходная задача сводится к построению в некоторой внутренней окрестности окружности $\mathbb{T}$ такого решения уравнения (8.22), которое экспоненциально убывает в направлении внутренней нормали к границе и удовлетворяет условию Дирихле $\left.v\right|_{s \in \Gamma}=f(s / \varepsilon)$, где $f \in H^{\alpha}(\mathbb{T})$ - граничная функция для исходной задачи. В том весьма частном случае, когда функция $G$ линейна по $\left(\rho, v, v_{\rho}\right)$ и не зависит от $\left(\varphi, v_{\varphi}\right)$, искомая асимптотика представима в виде суммы двух функций, одна из которых есть решение уравнения $\rho^{2} v_{\rho \rho}+\rho v_{\rho}+G\left(\rho, v, v_{\rho}\right)=0$, а другая строится согласно изложенному выше рецепту.

\section{§ 9. Задача Олейник-Темама об усреднении}

Здесь мы рассмотрим поставленную О. А. Олейник и Р. Темамом (см., в частности, [104]) задачу о построении при $\varepsilon \rightarrow 0$ равномерной вплоть до границы $\Gamma=\partial \Omega$ области $\Omega$ асимптотики для решения краевой задачи

$$
\Delta U_{\varepsilon}=0 \quad \text { в } \Omega,\left.\quad U_{\varepsilon}\right|_{s=(x, y) \in \Gamma}=f_{\varepsilon}(s), \quad \text { где } f_{\varepsilon}(s)=\left.F\left(\frac{x}{\varepsilon}, \frac{y}{\varepsilon}\right)\right|_{\partial \Gamma},
$$

а $F:(x, y) \mapsto F(x, y)$ - периодическая по каждому переменному функция, знакопостоянная на полупериоде, равном единице. В дополнение к замечанию 8.1, в котором приведен результат об асимптотике $U_{\varepsilon}$ в данном случае, дадим более явную конструкцию этой асимптотики для частного вида функции $F$.

9.1. Введем два экземпляра окружности, а именно:

$$
\mathbb{T}=\mathbb{T}_{\theta} \stackrel{\text { def }}{=}\left\{\psi=\mu e^{i \theta},|\psi|=1\right\} \quad \text { и } \quad \mathbb{T}=\mathbb{T}_{\sigma} \stackrel{\text { def }}{=}\left\{\zeta=\rho e^{i \sigma},|\zeta|=1\right\} .
$$


Рассмотрим ситуацию, когда для функции $f: \mathbb{T} \rightarrow \mathbb{R}$ найдется такой аналитический (и, для определенности, сохраняющий ориентацию) автоморфизм

$$
\sigma: \mathbb{T} \ni e^{i \theta} \mapsto e^{i \sigma(\theta)} \in \mathbb{T},
$$

а также такая константа $C \in \mathbb{R}$, что функция

$$
g: \theta \mapsto g(\theta) \stackrel{\text { def }}{=} f(\sigma(\theta))-C
$$

нечетна относительно, скажем, точки $\theta_{0}=0$, т. е. $g(-\theta)=-g(\theta)$. Представим $2 \pi$-периодические функции $\theta \mapsto \sigma(\theta)-\theta$ и $\theta \mapsto-\sigma^{\prime \prime}(\theta) / \sigma^{\prime}(\theta)$ соответственно в виде

$$
\begin{gathered}
\sigma(\theta)-\theta=f_{0}+\sum_{k \geqslant 1}\left(f_{k} \cos k \theta+g_{k} \sin k \theta\right), \\
-\frac{\sigma^{\prime \prime}(\theta)}{\sigma^{\prime}(\theta)}=\sum_{k \geqslant 1} k\left(c_{k} \cos k \theta+d_{k} \sin k \theta\right) .
\end{gathered}
$$

ЛЕмма 9.1. В некоторой окрестности $V_{\mathbb{T}}^{\theta}$ окружности $\mathbb{T}_{\theta}$ отображение

$$
\sigma \stackrel{\text { def }}{=} \zeta: V_{\mathbb{T}}^{\theta} \ni \psi=\mu e^{i \theta} \mapsto \zeta(\psi)=1+\int_{1}^{\psi} \exp \{a+i b\} d \psi \in \sigma\left(V_{\mathbb{T}}^{\theta}\right),
$$

в котором $a=a(\mu, \theta)$ u $b=b(\mu, \theta)$ представлены рядами

$a_{0}+\sum_{k \geqslant 1}\left[\left(\frac{d_{k}+g_{k}}{2} \mu^{k}+\frac{d_{k}-g_{k}}{2} \mu^{-k}\right) \cos k \theta-\left(\frac{c_{k}+f_{k}}{2} \mu^{k}+\frac{c_{k}-f_{k}}{2} \mu^{-k}\right) \sin k \theta\right]$,

где константа $a_{0}$ определяется из нормировки $\int_{0}^{2 \pi} e^{a(1, \theta)} d \theta=2 \pi, u$

$f_{0}+\sum_{k \geqslant 1}\left[\left(\frac{c_{k}+f_{k}}{2} \mu^{k}-\frac{c_{k}-f_{k}}{2} \mu^{-k}\right) \cos k \theta+\left(\frac{d_{k}+g_{k}}{2} \mu^{k}-\frac{d_{k}-g_{k}}{2} \mu^{-k}\right) \sin k \theta\right]$,

осуществляет такое однолистное отображсние $V_{\mathbb{T}}^{\theta}$ на $\sigma\left(V_{\mathbb{T}}^{\theta}\right)$, которое на $\mathbb{T}=\mathbb{T}^{\theta}$ coвnaдaem c $\sigma$, m.e. $\left.\sigma\right|_{\mathbb{T}}=\sigma$.

ДокАЗАТЕЛЬСтво. При $\mu=1$ отображение (9.6) должно совпадать с заданным автоморфизмом $\sigma$ окружности $\mathbb{T}=\left\{\left|e^{i \theta}\right|=1\right\}$, т. е. должно быть выполнено условие

$$
\left.\left|\frac{d \zeta}{d \psi}\right|\right|_{\psi=e^{i \theta}}=\sigma^{\prime}(\theta)
$$

Это эквивалентно тому, что $\left.a(\mu, \theta)\right|_{\mu=1}=\ln \sigma^{\prime}(\theta)$. Отсюда (ср. с [48], [57]) следует

$$
\left.b_{\mu}^{\prime}(\mu, \theta)\right|_{\mu=1}=-\left.a_{\theta}^{\prime}(\mu, \theta)\right|_{\mu=1}=-\left[\ln \sigma^{\prime}(\theta)\right]_{\theta}^{\prime}=-\frac{\sigma^{\prime \prime}(\theta)}{\sigma^{\prime}(\theta)} .
$$

Остается заметить, что угловая функция $N$ для окружности есть тождественное отображение (угол наклона нормали к оси $x$ в любой точке окружности это полярный угол этой точки). Поэтому

$$
\left.b(\mu, \theta)\right|_{\mu=1}=\sigma(\theta)-\theta .
$$


Тем самым, аналитическая функция $a+i b$, задаваемая формулами (9.7)-(9.8), определяет в окрестности $V_{\mathbb{T}}^{\theta}$ единичной окружности $\mathbb{T}$ отображение (9.6) с требуемыми свойствами. Лемма 9.1 доказана.

По построению, композиция $f \circ \sigma$ функции $f \in H^{\alpha}(\mathbb{T})$ с аналитическим автоморфизмом $\sigma: \mathbb{T} \rightarrow \mathbb{T}$ есть нечетная функция с точностью до аддитивной константы. Рассмотрим однолистное отображение

$$
z \circ \sigma: V_{\mathbb{T}}^{\theta} \ni \psi=\mu e^{i \theta} \mapsto z(\sigma(\psi)) \in V_{\Gamma}^{\sigma} \stackrel{\text { def }}{=} z\left(\sigma\left(V_{\mathbb{T}}\right)\right) .
$$

Оно конформно и $\sigma$-изометрично отображает окружность $\mathbb{T}_{\theta}$ в аналитическую кривую $\Gamma$.

Обозначим через $\pi_{\Gamma}^{\sigma}$ поле трансверсалей к $Г$, которое является образом нормалей к окружности $\mathbb{T}_{\theta}$ при отображении (9.11). Поле $\pi_{\Gamma}^{\sigma}$ позволяет получить полезную информацию о графике точной асимптотики $u_{\varepsilon}$. А именно, согласно предыдущим построениям, справедлива следующая теорема.

ТЕОрема 9.1 [56]. Пусть выполнены предположения, представленные в формулах (9.2) и (9.3). Тогда линии уровня $u_{\varepsilon}(x, y)=C$ функции $u_{\varepsilon}$ (для указанной в (9.3) константы $C$ ) вкладываются в поле $\pi_{\Gamma}^{\sigma}$ трансверсалей $\kappa$ Г.

Заметим, что в случае кусочно аналитической границы знание поля $\pi_{\Gamma}$ (или в более общей ситуации поля $\pi_{\Gamma}^{\sigma}$ ) позволяет локализовать и выявить особенности асимптотики в окрестности угловых точек границы.

9.2. Перейдем теперь к намеченному в заметке [56] построению асимптотического приближения для решения задачи Дирихле

$$
\Delta U_{\varepsilon}=0 \quad \text { в } \Omega \Subset \mathbb{R}^{2},\left.\quad U_{\varepsilon}\right|_{\partial \Omega}=F\left(\frac{x}{\varepsilon}, \frac{y}{\varepsilon}\right)
$$

в случае аналитической кривой $\Gamma=\partial \Omega$ (см. рис. 16, а)). Ограничимся здесь случаем двузначной функции $F$, которая $(1,1)$-периодична и нечетна по каждому переменному. Пусть для определенности область $\Omega$ односвязна, а длина $|\Gamma|$ кривой $\Gamma$ равна $2 \pi$. Построение базируется на приведенных выше формулах (8.14), (9.6), задающих

1) изометрическое конформное отображение окружности $\mathbb{T}_{\sigma}$ на кривую $\Gamma$;

2) конформное отображение $\sigma=\zeta: \mathbb{T}_{\theta} \ni \psi \mapsto \zeta(\psi) \in \mathbb{T}_{\sigma}$, которое на $\mathbb{T}_{\theta}$ совпадает с заданным аналитическим автоморфизмом $\sigma: \mathbb{T}_{\theta} \rightarrow \mathbb{T}_{\sigma}$.

Построение асимптотики осуществляется следующим образом. При заданных $\varepsilon$ и аналитической кривой $\Gamma$ (см. рис. 16, а)) на $\Gamma$ фиксируется след функции $(x, y) \mapsto F(x / \varepsilon, y / \varepsilon)$. Развертка этого следа, параметризованная точками $\sigma_{k}$ (см. рис. $\left.\left.16, \mathrm{~d}\right)\right)$, переносится в виде графика функции $\sigma \mapsto f_{\varepsilon}(\sigma)$ на окружность $\mathbb{T}_{\sigma}$ (см. рис. $\left.16, \mathrm{~b}\right)$ ). Отметим, что образом окружности $\mathbb{T}_{\sigma}$ при отображении (8.14) является кривая Г. В предположении, что

$$
\max _{k}\left(\sigma_{k}-\sigma_{k-1}\right) \leqslant C \varepsilon, \quad \text { где } C=O(1) \text { при } \varepsilon \rightarrow 0,
$$

сначала строится полином $\theta \mapsto \sigma(\theta)$, принимающий значение $\sigma_{k}$ в соответствующей точке $\theta_{k}$ (см. рис. $\left.\left.\left.16, \mathrm{~d}\right), \mathrm{e}\right)\right)$. Затем строится решение $v_{\varepsilon}$ задачи Дирихле для уравнения Лапласа в диске $D_{\theta}$ (см. рис. 16, с)) с данными Дирихле, представленными на рис. 16, е) в виде развертки графика функции $f(\theta / \varepsilon)$. Наконец, 


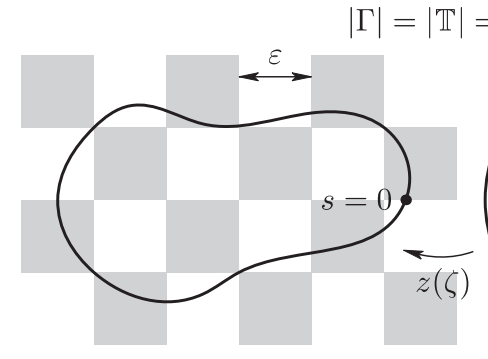

a)

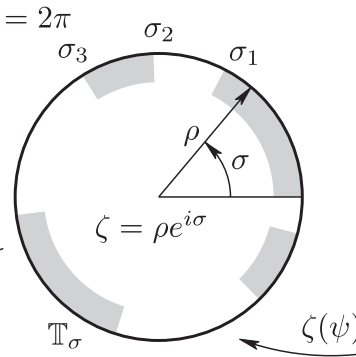

b)

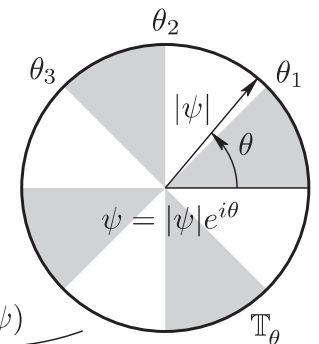

c)

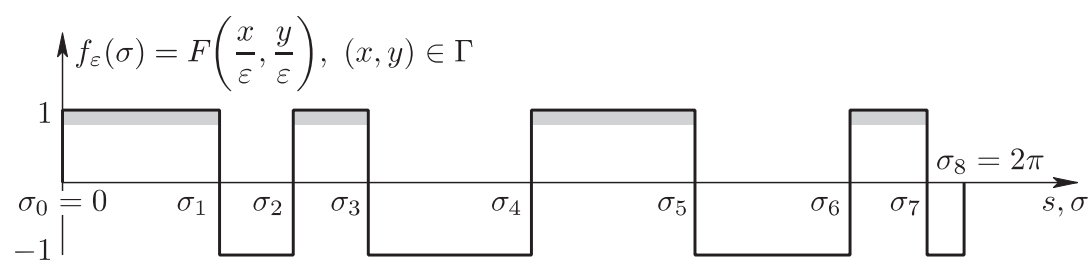

d)

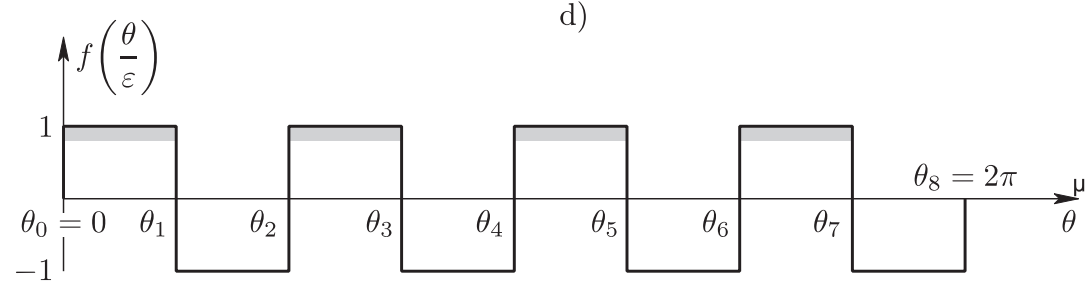

e)

Рис. 16

построенное решение $v_{\varepsilon}$ трансформируется в искомую асимптотику с помощью $\sigma$-отображения (9.6), следуя теореме 8.1.

\section{$\S 10$. Экспоненциально точная асимптотика гармонической функции в области с сильно гофрированной границей}

Коснемся вопроса построения асимптотики решения задачи

$$
\left.\Delta U_{\varepsilon}\right|_{\Omega_{\varepsilon}}=0,\left.\quad\left(U_{\varepsilon}+\lambda \frac{\partial U_{\varepsilon}}{\partial \nu}\right)\right|_{s \in \Gamma_{\varepsilon}}=f(s), \quad f \in H^{\beta}\left(\Gamma_{\varepsilon}\right), \quad \beta \geqslant-\frac{1}{2}-\operatorname{sign} \lambda,
$$

в области $\Omega_{\varepsilon}$ (скажем, односвязной) с сильно гофрированной аналитической границей $\Gamma_{\varepsilon}$, угловая функция которой представима в виде

$$
N_{\varepsilon}:[0,2 \pi] \ni s \mapsto N_{\varepsilon}(s)=N(s)+\sum_{k=1}^{K} \varepsilon^{k}\left(f_{k} \cos \frac{k s}{\varepsilon}+g_{k} \sin \frac{k s}{\varepsilon}\right),
$$

где $1 / \varepsilon=M \in \mathbb{Z}$. Формула (10.2) означает, что длина кривой $\Gamma_{\varepsilon}$ равна $2 \pi$, а угол между осью $x$ и внешней нормалью к $\Gamma_{\varepsilon}$ в точке, соответствующей натуральному параметру $s$ этой кривой, равен $N_{\varepsilon}(s)=N(s)+n_{\varepsilon}(s)$, где $N-$ угловая функция кривой $\Gamma$, фигурирующая в формулах (8.9) и (8.15). 
Рассмотрим однолистное отображение $z_{\varepsilon}$, определенное по формуле (8.14), в которой функция $A+i B$, заданная формулами (8.16)-(8.17), заменена на функцию $A^{\varepsilon}+i B^{\varepsilon}=(A+i B)+\left(a^{\varepsilon}+i b^{\varepsilon}\right)$, где

$$
\begin{aligned}
& a^{\varepsilon}(\rho, \beta)+i b^{\varepsilon}(\rho, \beta) \\
& \quad=\sum_{k=1}^{K}\left(\frac{\rho^{2 k}-\varepsilon^{2 k}}{2 \rho^{k}}\left[g_{k} \cos k \beta-f_{k} \sin k \beta\right]+i \frac{\rho^{2 k}+\varepsilon^{2 k}}{2 \rho^{k}}\left[f_{k} \cos k \beta+g_{k} \sin k \beta\right]\right) .
\end{aligned}
$$

Пусть $\zeta_{\varepsilon}: V_{\Gamma_{\varepsilon}} \stackrel{\text { def }}{=} z_{\varepsilon}\left(V_{\mathbb{T}}\right) \ni z \mapsto \zeta_{\varepsilon}(z) \in V_{\mathbb{T}}$ - отображение, обратное к $z_{\varepsilon}$ (ср. (8.8)). Возьмем, как и выше, две кольцевые области $V_{\mathbb{T}}^{0}$ и $V_{\mathbb{T}}^{1} \subset V_{\mathbb{T}}^{0} \subset V_{\mathbb{T}}$, определяемые радиусами $\rho_{k}$ малой окружности кольца $V_{\mathbb{T}}^{k}=\left\{\rho_{k}<\rho<1\right\}$, и рассмотрим их прообразы $V_{\Gamma_{\varepsilon}}^{k}=z_{\varepsilon}\left(V_{\mathbb{T}}^{k}\right) \subset V_{\Gamma_{\varepsilon}}$ при отображении $\zeta_{\varepsilon}: V_{\Gamma_{\varepsilon}} \rightarrow V_{\mathbb{T}}$. Пусть $\chi \in C^{\infty}\left(\Omega_{\varepsilon}\right)$ - срезающая функция такая, что $\chi=1$ в $V_{\Gamma_{\varepsilon}}^{1}$ и $\chi=0$ вне $V_{\Gamma_{\varepsilon}}^{0}$.

Обозначим через $v$ решение задачи (10.1) для случая, когда область $\Omega_{\varepsilon}$ есть единичный диск. Тогда функция $u_{\varepsilon}=\chi v \circ \zeta_{\varepsilon}$ есть точная асимптотика решения задачи (10.1), т. е. для нее справедлива оценка (8.2).

Некоторые открытые вопросы. 1. Для каких достаточно широких подклассов функций $f$ из $H^{\beta}(\mathbb{T})$, где $\beta \geqslant-1 / 2$, выполнены предположения, представленные в формулах (9.2) и (9.3)? Какой можно предложить эффективный способ нахождения соответствующего отображения $\sigma$ в каждом из этих подклассов?

2. Каков аналог теоремы 8.1 для случая, когда $\lambda \neq 0$ в условии (8.1)?

\section{Дополнение 1. Токамаки и взаимодействие солитонов}

Основными элементами токамака - ТОроидальной КАмеры с МАгнитными Катушками (см. рис. 17) - являются вакуумная тороидальная камера, трансформатор и катушки тороидального магнитного поля. Камера, в которую запускают водород, например, его изотопы дейтерий и тритий, служит также вторичной обмоткой (катушкой) трансформатора. Через первичную обмотку трансформатора пропускают большой импульсный (переменный) ток, который возбуждает тороидальное (направленное вдоль большой окружности тора) вихревое электрическое поле. Оно осуществляет пробой (поджог) водорода, превращая его в ионно-электронный газ, т. е. в плазму. B DT-реакции синтеза одного ядра дейтерия и одного ядра трития образуется атом гелия, один нейтрон и освобождается (за счет дефекта масс) энергия порядка $7 \cdot 10^{-18}$ квт час (1 кг DT-топлива эквивалентен 250 кг обогащенного урана). Проблема теплоизоляции высокотемпературной плазмы решается посредством магнитного поля, благодаря которому плазма как бы подвешена в вакуумном пространстве камеры токамака. Индуцированный в плазме продольный (вдоль большой окружности тора) электрический ток создает магнитное поле, силовые линии которого ортогональны большой окружности тора и, будучи концентрическими, имеют полюс. Поэтому это поле называется полоидальным. Оно и тороидальное магнитное поле образуют результирующее магнитное поле, силовые линии которого спиралями обматывают тор. Вдоль них движутся (в магнитогидродинамическом приближении) заряженные частицы плазмы, т. е. ионы 
Железный сердечник трасформатора
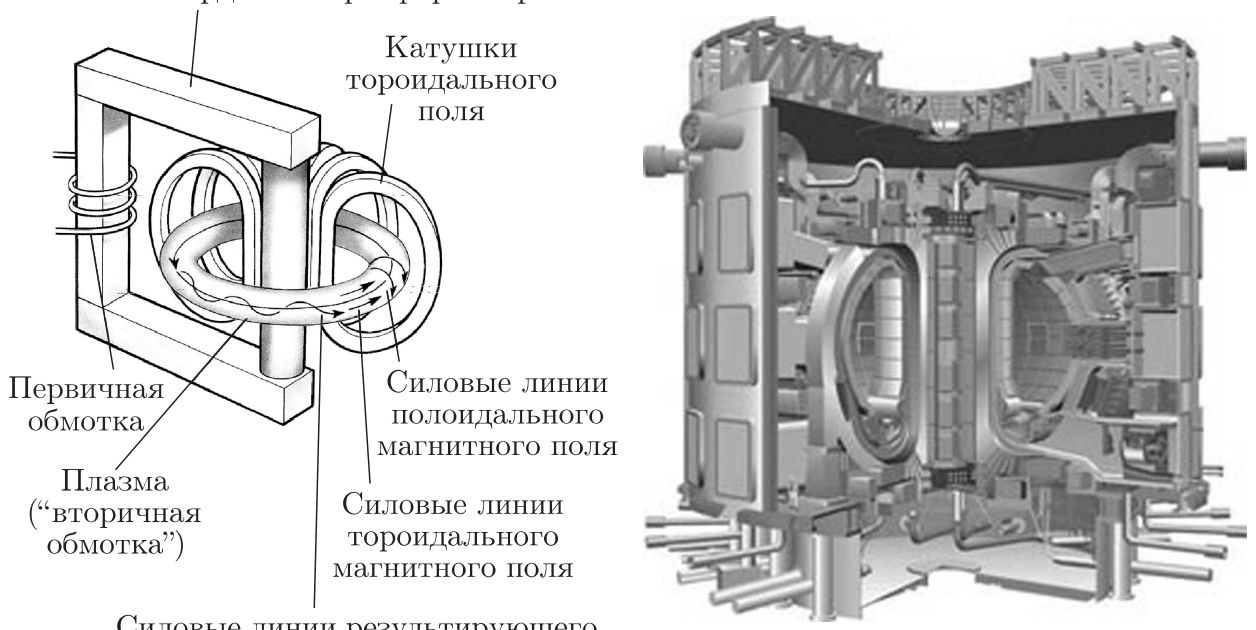

Силовые линии результирующего магнитного поля (шаг винта преувеличен)

Рис. 17. Схема токамака и вид реактора ИТЭР (лат. iter - путь) этого международного (ЕС, Россия, США, Япония и впоследствии присоединившиеся к проекту Индия, КНР и Республика Корея) экспериментального термоядерного реактора (International Thermonuclear Experimental Reactor) [105], [106], который строится в 60 км северо-восточнее Марселя в исследовательском центре ядерной энергетики Кадараш (Cadarache). Большой/малый радиус плазменного разряда равен $6.2 \mathrm{~m} / 2 \mathrm{м}$, объем плазмы $837 \mathrm{~m}^{3}$. Запланированная плотность плазмы невысока (в $10^{6}$ меньше плотности атмосферного воздуха). Однако ее будет достаточно, чтобы впервые достичь уровня выделяемой энергии, необходимого для самоподдержания реакции синтеза, поскольку разряд должен длиться около 7 минут, а расчетная температура плазмы составляет $150 \div 200$ млн. градусов (что на порядок выше температуры в центре Солнца).

и оторванные от них электроны, изолируясь тем самым от соприкосновения с камерой. Тороидальное поле играет к тому же главную роль в подавлении магнитогидродинамической неустойчивости плазмы.

Равновесие плазмы как в магнитных ловушках [107], к которым относятся токамаки, так и в астрофизике [108], [109] описывается уравнением Грэда-Шафранова, называемого иногда SLSG-Equation. Это уравнение было получено В. Д. Шафрановым в начале 50-х годов и независимо от него Грэдом. После рассекречивания программ управляемого термоядерного синтеза (чему способствовал доклад в 1956 г. И.В.Курчатова в английском атомном центре Harewell) это уравнение появилось в открытых публикациях сначала в работах В. Д. Шафранова [10], [110], а затем в работах Люста-Шлютера [111] и Грэда-Рубина [112].

В недавней работе [40] рассмотрена более реалистичная модель плазмодинамики и плазмостатики, в которой каждая из двух “жидкостей” (электроны и ионы) представлена своим комплектом гидродинамических уравнений. Эта мо- 
дель сводится к формально одножидкостной, которая имеет гиперболический тип для сильно разреженной плазмы и эллиптический для плотной. Уравнение Грэда-Шафранова является предельным и вырожденным случаем полученной в [40] системы. Ее динамический вариант интересен в том числе и тем, что обладает (как показали численные эксперименты) эффектами взаимодействия солитонов, характерными для уравнений типа Кортевега-де Фриза.

\section{Дополнение 2. Сравнение функционально-геометрического метода и метода годографа}

Вслед за пионерской работой Гельмгольца [1] о форме вытекающей из щели плоской струи было решено (см., в частности, [97], [113], [114]) немало задач этого класса с помощью метода годографа в интерпретации, восходящей к Кирхгофу [43] и получившей развитие в работах М. Планка [115], Н. Е. Жуковского [49], С. А. Чаплыгина [116], Т. Леви-Чивиты [117] и многих других авторов. Метод годографа применим в случае, когда может быть явно охарактеризована область $\mathscr{K}=d z /\left.d w\right|_{w \in Q}$. Назовем ее областью Кирхгофа. Она очевидным образом связана с так называемой областью годографа $\mathscr{H} \stackrel{\text { def }}{=} d w /\left.d z\right|_{z \in \Omega}$ (от гр. hodos - путь), т. е. множеством на комплексной плоскости, которое кончик вектора комплексно-сопряженной скорости $\overline{\nabla u}: \Omega \ni z \mapsto d w / d z \in \mathscr{H}$ проходит, двигаясь по пути, определяемому точкой $(x, y)$, пробегающей всю искомую область течения. При заданной области $\mathscr{K}=d z /\left.d w\right|_{w \in Q}$ разыскиваемая область $\Omega=\left\{z(w)=\int \frac{d z}{d w} d w, w \in Q\right\}$ может быть найдена, ибо $d z / d w=\varkappa(w)$, где отображение $\varkappa: Q \rightarrow \mathscr{K}$ строится с помощью однолистных отображений $k$ и $q$ в следующей диаграмме:

$$
\begin{aligned}
& \frac{d z}{d w} \in \underset{\uparrow}{ } \in \underset{k}{\longrightarrow} \mathbb{C}_{+} \ni k(\varkappa(w)) \\
& w \in Q \stackrel{q}{\longrightarrow} \mathbb{C}_{+} \ni q(w)
\end{aligned}
$$

Согласно этой диаграмме, $\varkappa(w)=k^{-1}\left(\frac{a q(w)+b}{c q(w)+d}\right)$, а коэффициенты $a, b, c$ и $d$ дробно-линейного автоморфизма полуплоскости $\mathbb{C}_{+}$находятся (с точностью до пропорциональности) из соответствий, заданных отображениями $k$ и $q$. В этом и заключается суть того метода годографа, который ассоциируется с именами Кирхгофа и его последователей. (Нечто иное, связанное с преобразованием Лежандра и его обобщениями, но относящееся тоже к hodos grapho, также называют методом годографа [26], [103].)

Итак, в тех простейших случаях, когда область Кирхгофа может быть описана явно, задачу построения искомого отображения $d z / d w$ можно свести к построению однолистного отображения $\varkappa: Q \rightarrow \mathscr{K}$. Это то, чем занимались классики. Но в общем случае область $\mathscr{K}$ (и, соответственно, область годографа) не поддается явному описанию, поскольку она характеризуется (например, как 
в (1.39)-(1.41)) решением, вообще говоря, нелинейной задачи Римана-Гильберта с нелинейными ограничениями для функции Гельмгольца-Кирхгофа

$$
A+i B: Q=w(\Omega) \ni w=u+i v \mapsto A(u, v)+i B(u, v) \stackrel{\text { def }}{=} \ln \frac{d z}{d w} .
$$

Эта функция решает исходную задачу нахождения искомой области течения и его скорости, ибо

$$
\Omega=\left\{z(w)=z_{0}+\int_{w_{0}}^{w} e^{A(u, v)+i B(u, v)} d w, w \in Q, z_{0}=z\left(w_{0}\right)\right\}
$$

a

$$
\vec{V}(z)=\left.e^{-A(u, v)+i B(u, v)}\right|_{u+i v=w(z)} .
$$

Именно такая общая ситуация, в том числе та, когда область $Q=w(\Omega)$ не является фиксированной и/или односвязной, представляет особый интерес и значимость. Именно ей посвящен обзор.

В чем же различие между функционально-геометрическим методом и методом годографа? Каждый из них дополняет ГК-метод (представленный в п. 1.4 на с. 19). Однако их возможности различны. Метод годографа в интерпретации, восходящей к Кирхгофу, позволяет в ряде случаев получить решение возникающей задачи Римана-Гильберта в так называемой замкнутой форме, т. е. в виде интеграла, а порой и явной формулы, содержащей лишь элементарные функции. Это дает возможность получить в этих случаях качественный или даже числовой ответ для исходной задачи. Следует сказать, что раньше этот технический прием был практически единственным средством, позволяющим завершать анализ некоторых ГК-задач. Комментарии по поводу его модификаций в книгах по гидродинамике мало отличаются от тех, что имеются в известном учебнике [114]: “Для решения основной задачи отображения области течения $z$ на плоскость $\zeta$ Леви-Чивита предложил рассматривать вместо переменной Кирхгофа $\frac{1}{d w / d z}$ и переменной Жуковского $\ln \frac{d w /\left.d z\right|_{\infty}}{d w / d z}$ комплексную переменную $i \ln d w / d z$ ". Однако такая “устаревшая” техника (метод Кирхгофа, метод Жуковского-Митчеля, метод Леви-Чивиты,...) позволяет только в сравнительно простых случаях преодолевать трудности, связанные с соответствующей ГК-методу краевой задачей, являющейся в общем случае весьма специфической нелинейной задачей Римана-Гильберта с нелинейными ограничениями. Если учесть при этом, что упомянутая техника долгое время воспринималась (даже Чаплыгиным; см. примечания к русскому переводу статьи Гельмгольца [1]) как главное в развитии исходных идей Гельмгольца-Кирхгофа и затушевывала их базовую суть, то станет понятным, почему базовые идеи Гельмгольца и Кирхгофа как бы затерялись за их техническим обрамлением. А если учесть, что эта техника (т. е. метод годографа) абсолютно уступает современным вычислительным средствам и методам численного анализа в вопросе доведения решения задачи "до числа", то станет понятной и причина часто встречающегося в наши дни мнения, будто идеи Гельмгольца-Кирхгофа уже исчерпали свои возможности.

Цель данного обзора - доказать обратное: потенциал базовых идей Гельмгольца и Кирхгофа, заключенных в ГК-методе, далеко не исчерпан [118]. 
Нужно лишь снять с них оковы техники конформных отображений метода годографа и дополнить их взаимосвязанными функциональными и геометрическими рассмотрениями задачи Римана-Гильберта, соответствующей ГК-методу (не ограничиваясь только функциональными методами, скажем, методами сингулярных интегральных уравнений).

Отметим в связи с этим некоторые выявленные в обзоре возможности функционально-геометрического метода, позволившие установить ряд результатов и фактов, которые не удавалось получить или выявить иными способами.

При доказательстве теорем 1.1 и 1.7 применялись конечномерные аппроксимации $K_{m}^{\lambda}=I-F_{m}^{\lambda}: \mathscr{D}_{m} \rightarrow \mathbb{R}^{m}$. Они были напрямую связаны с геометрическими характеристиками контура $\Gamma$ (с его самонепересекаемостью), а не с требованием равномерной сходимости $K_{m}^{\lambda} \rightarrow K^{\lambda}$. Именно это позволило снять обременительное ограничение компактности отображения $K^{\lambda}: \mathscr{D} \rightarrow X$, которое при непосредственном применении теоремы Лере-Шаудера в пространстве $X$ гёльдеровых функций (возникающих в случае криволинейного контура Г) приводит к очень жестким ограничениям на геометрию Г. Геометрическая составляющая функционально-геометрического метода позволила также сравнительно легко завершить доказательство теорем существования 1.1 и 1.7 при переходе от полигональных контуров $\Gamma_{m}$ к спрямляемому контуру Г, благодаря геометрически очевидной оценке (1.77).

Хорошо известны трудности построения численного решения задачи Коши для уравнения Лапласа. Они связаны с неустойчивостью решения относительно возмущений начальных данных, неизбежных при их задании даже с машинной точностью. Функционально-геометрическое же рассмотрение этого вопроса, проведенное в $\S 2$, позволяет получить численное решение этой задачи по явным формулам, обойдя отмеченные трудности.

Задачи обтекания - традиционная тематика, связанная с применением метода годографа. Однако и здесь выявилась бо́льшая эффективность функционально-геометрического метода. Удалось [94], [96] наметить подход в решении сложных задач граничного управления при нестационарном обтекании препятствия. Такие задачи напрямую не под силу даже для современных вычислительных средств.

Функционально-геометрический метод позволил также, как показано в главе IV, строить экспоненциально точные высокочастотные асимптотики гармонических функций, причем в наиболее сильной норме, в которой ограничено решение.

Наконец, благодаря функционально-геометрическим построениям был найден тот математический объект, который ответствен за "пальцевую" неустойчивость Кочиной-Саффмана-Тейлора. Важную роль здесь сыграл анализ интегро-дифференциального уравнения (3.9). Оно было выведено также с помощью функционально-геометрического метода.

\section{Дополнение 3. "Течение Хиле-Шоу", "Multigrid-method" и пр.}

В этом дополнении речь пойдет о том, что касается всего нашего математического сообщества. Импульсом к написанию этого дополнения послужила причина, указанная в сноске на с. 48). 
До 1968 г. имя английского морского инженера Генри Хиле-Шоу (1854-1941) изредка упоминалось в научной литературе. Но вот наступил 1968 г. и появилась публикация Б. У. Томсона [119], который приехал в Лондон из Мельбурна писать диссертацию по теме ячейки Хиле-Шоу. Б. У. Томсон и стал (это признает сам Дж. Р. Окендон в [120]) автором термина "течение Хиле-Шоу". В [119] этот термин употреблен почти случайно, всего один лишь только раз (это не цитата из песни, это действительно так). И тем не менее, одного этого случайного паса австралийского "легионера" было достаточно для опытной британской команды, возглавляемой Дж. Р. Окендоном, чтобы превратить этот термин в "бренд" (это же не какая-то ячейка в каком-то приборе, а течение!). События разворачивались просто гениально (чуть ниже читатель поймет, почему я об этом так подробно пишу). В 1972 г. вышла работа С. Ричардсона [85], где этот термин впервые был вынесен в заглавие статьи. Уже это привлекло внимание (что это за течение?). Но самое главное, в статье были получены прекрасные результаты. Об одном из них было сказано при доказательстве теоремы 3.3. Почин Ричардсона подхватила вся команда Окендона-Хауисона. В результате термин “течение Хиле-Шоу" стал ключевым словом для тысяч публикаций (см., в частности, [70]). Кроме того, попутно имя еще одного англичанина стало знаковым.

Все это была преамбула, цель которой подчеркнуть контраст с тем, что вызывает (выражаясь дипломатическим языком) определенную озабоченность и ради чего я пишу это дополнение. Речь не столько о скромности многих наших российских классиков, сколько о копировании (а потому и поддержке) тех вводимых на Западе названий важнейших методов, теорем, лемм, понятий, которые не отражают существенный, а зачастую основополагающий вклад в эти субстанции наших первопроходцев. Почему, к примеру, нужно поддерживать употребление термина "Multigrid-method", a не внедрить в численный анализ термин "метод Федоренко-Бахвалова". Можно сказать и так: "Fedorenko's multigrid-method". Ведь говорим, например, об альтернирующем методе Шварца [121]. Говорим же (и совершенно обоснованно) об эллиптических и гиперболических системах по Петровскому, об эллиптических системах по Дуглису-Ниренбергу, гиперболических системах по Лере-Волевичу. Но много ли тех (даже из российских математиков), кто назовет имя того, кому Математика обязана как самим понятием "сильно эллиптические системы", так и основополагающими для них результатами? Если студент, пожелавший найти ответ на этот вопрос, заглянет, скажем, в предметный указатель популярного у нас учебника [122] и откроет соответствующую страницу, то он увидит лишь имя Гординга. Жаль, что студент не узнает, что первоначально усиленное неравенство Гординга для функций с малым носителем было доказано М. И. Вишиком для введенных им сильно эллиптических систем. К чести Гординга, сам он признавал принципиальный вклад М. И. Вишика в то, что называется неравенством Гординга. А что знают некоторые "продвинутые" зарубежные математики о И. Г. Петровском? Слава Богу, хоть что-то (см. по этому поводу [123]), ибо есть термин " $2 b$-параболические по Петровскому системы”. Так почему же (вопреки почину Лере и Лионса [124]) нужно опускать в соответствующем термине имя того, кто первый получил важнейшие результаты для введенных им квазилинейных сильно эллиптических систем, называемых (после аксиоматизации 
их свойств) "p-монотонными операторами" и ассоциируемых нередко, особенно на Западе, лишь с именами Браудера и Минти? Почему надо употреблять термин "лемма Лакса-Мильграма", покорно “забывая" (в противовес мнению самого Лакса) о первом (т. е. М. И. Вишике), кто установил соответствующий результат? Не лучше ли говорить о ВЛМ-лемме? Речь не о том, чтобы продолжить такого сорта вопросы (это сделать легко). Речь об уважении к авторам важнейших понятий, результатов, методов и о необходимости отстаивать их приоритет и, в частности, приоритет соотечественников, упоминая их имена в соответствующих названиях теорем, лемм, методов, понятий. Конечно, не все так печально. У всех на слуху уравнение Гельфанда-Левитана, принцип максимума Понтрягина, индекс Арнольда-Маслова и т. д. Но надо исправлять допущенные промахи, особенно в отношении авторов, себя не рекламирующих.

\section{Список литературы}

[1] H. Helmholtz, "Über discontinuirliche Flüssigkeitsbewegungen", Monatsberichte der Königlich Preußischen Academie der Wissenschaften zu Berlin, 1868, 215-228; pyc. пер. в: H. Helmholtz, Два исследования по гидродинамике. I: О вихревом движе-

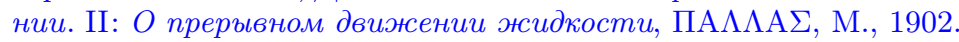

[2] A. S. Demidov, "The form of a steady plasma subject to the skin effect in a Tokamak with non-circular cross-section", Nuclear Fusion, 15 (1975), 765-768.

[3] А. С. Демидов, "Об одной задаче со свободной границей в теории равновесной плазмы”, Тр. сем. им. И. Г. Петровского, 4 (1978), 65-82.

[4] A.S. Demidov, "Configurations du plasma stationnaire équilibré", Free boundary problems, vol. I (Pavia, 1979), Ist. Naz. Alta Mat. Francesco Severi, Rome, 1980, 467-485.

[5] А. С. Демидов, "Об обратной задаче для уравнения Грэда-Шафранова с аффинной правой частью”, УМH, 55:6 (2000), 131-132; англ. пер.: А. S. Demidov, "On the inverse problem for the Grad-Shafranov equation with affine right-hand side", Russian Math. Surveys, 55:6 (2000), 1141-1142.

[6] A. S. Demidov, "On the inverse problem for the Grad-Shafranov equation with affine right-hand side", Russ. J. Math. Phys., 17:2 (2010) (to appear).

[7] A.S. Demidov, M. Moussaoui, "An inverse problem originating from magnetohydrodynamics", Inverse Problems, 20:1 (2004), 137-154.

[8] A. S. Demidov, V.V. Savelyev, "Essentially different current distributions in the inverse problem for the Grad-Shafranov equation", Russ. J. Math. Phys., 17:1 (2010) (to appear).

[9] M. Vogelius, "An inverse problem for the equation $\Delta u=-c u-d$ ", Ann. Inst. Fourier (Grenoble), 44:4 (1994), 1181-1209.

[10] V.D. Shafranov, "On equilibrium magnetohydrodynamic configurations", Terzo Congresso Internazionale sui Fenomeni d'Ionizzazione nei Gas (Venezia, 1957), Milano, 1957, 990-997.

[11] G. G. Stokes, "Mathematical proof of the identity of the stream lines obtained by means of a viscous film with those of a perfect fluid moving in two dimensions", Brit. Ass. Rep., 1898, 143-144.

[12] Л. С. Лейбензон, Нефтепромысловая механика, ч. II, Нефтеиздат, М., 1934.

[13] H. S. Hele-Shaw, "The flow of water", Nature, 58 (1897), 467-468.

[14] П. Я. Кочина, А. Р. Шкирич, "К вопросу о перемещении контура нефтеносности (эксперимент)", Изв. АН СССР, отд. технич. наук, 1954, № 11, 105-107.

[15] P. G. Saffman, G. I. Taylor, "The penetration of a fluid into a porous medium of Hele-Shaw cell containing a more viscous liquid", Proc. Roy. Soc. London. Ser. A, 245:1242 (1958), 312-329. 
[16] A. N. Gorban', A. M. Gorlov, V. M. Silantyev, "Limits of the turbine efficiency for free fluid flow", J. Energy Resources Technology, 123:4 (2001), 311-317.

[17] A. Gorban', M. Braverman, V. Silantyev, "Modified Kirchhoff flow with a partially penetrable obstacle and its application to the efficiency of free flow turbines", Math. Comput. Modelling, 35:13 (2002), 1371-1375.

[18] A. M. Gorlov, "Helical turbine for the Gulf Stream", Marine Technology, 35:3 (1998), $175-182$.

[19] Д.В. Маклаков, Нелинейные задачи гидродинамики потенииальных течений с неизвестными границами, Янус-К, М., 1997.

[20] А. С. Демидов, Л.Е. Захаров, "Прямая и обратная задачи в теории равновесия плазмы", УМН, 29:6 (1974), 203.

[21] J. Blum, H. Buvat, "An inverse problem in plasma physics: the identification of the current density profile in a tokamak", Large-scale optimization with applications, Part I (Minneapolis, MN, 1995), IMA Vol. Math. Appl., 92, Springer, New York, 1997, 17-36.

[22] V.D. Pustovitov, "Magnetic diagnostics: General principles and the problem of reconstruction of plasma current and pressure profiles in toroidal systems", Nuclear Fusion, 41:6 (2001), 721-730.

[23] L. E. Zakharov, J. Lewandowski, L. E. Foley, F. M. Levinton, H. Y. Yuh, V. Drozdov, D. C. McDonald, "The theory of variances in equilibrium reconstruction", Physics of Plasma, 15:9 (2008), 092503.

[24] J. Mossino, "A priori estimates for a model of Grad-Mercier type in plasma confinement", Applicable Anal., 13:3 (1982), 185-207.

[25] J. Mossino, "Isoperimetric inequalities and nonexistence resultat for the Grad-Shafranov equations", Nonlinear Anal., 11:2 (1987), 231-244.

[26] А. Фридман, Вариационные приниипы и задачи со свободными границами, Наука, М., 1990; пер. с англ.: А. Friedman, Variational principles and free-boundary problems, Pure Appl. Math., Wiley, New York, 1982.

[27] H. Berestycki, H. Brezis, "Sur certains problèmes de frontière libre", C. R. Acad. Sci. Paris Sér. A-B, 283:16 (1976), 1091-1094.

[28] H. Berestycki, H. Brezis, "On a free boundary problem arising in plasma physics", Nonlinear Anal., 4:3 (1980), 415-436.

[29] R. Temam, "A non-linear eigenvalue problem: The shape at equilibrium of a confined plasma", Arch. Ration. Mech. Anal., 60:1 (1975), 51-73.

[30] R. Temam, "Remarks on a free boundary problem arising in plasma physics", Comm. Partial Differential Equations, 2:6 (1977), 563-585.

[31] L. A. Caffarelli, G. Spruck, "Convexity properties of solutions of some classical variational problems", Comm. Partial Differential Equations, 7:11 (1982), 1337-1379.

[32] D. Kinderlehrer, L. Nirenberg, "Regularity in free boundary problems", Ann. Scuola Norm. Sup. Pisa Cl. Sci. (4), 4:2 (1977), 373-391.

[33] D. Kinderlehrer, J. Spruck, "The shape and smoothness of stable plasma configurations", Ann. Scuola Norm. Sup. Pisa Cl. Sci. (4), 5:1 (1978), 131-148.

[34] И. И. Данилюк, Об интегральных функционалах с переменной областью интегрирования, Тр. МИАН, 118, Наука, М., 1972.

[35] П. Н. Вабищевич, Л. М. Дектярев, Ю. Ю. Пошехонов, Численное решение прямой и обратной задачи МГД-равновесия с поверхностным током, Препринт № 9 ИПМ им. М. В. Келдыша, М., 1979.

[36] A. Acker, "On the qualitative theory of parametrized families of free boundaries", J. Reine Angew. Math., 393 (1989), 134-169.

[37] A. Beurling, "On free-boundary problems for the Laplace equation", Semin. on Analytic Functions, N.Y. Inst. Adv. Study, V.I, 1957, 248-263. 
[38] Y. Suzuki, H. Yamada, N. Nakajima, K. Watanabe, Y. Nakamura, T. Hayashi, "Theoretical considerations of doublet-like configuration in LHD", Nuclear Fusion, 46:1 (2006), 123-132.

[39] A. S. Demidov, "Sur la perturbation 'singulière' dans un problème à frontière libre", Singular perturbations and boundary layer theory (Lyon, 1976), Lecture Notes in Math., 594, Springer, Berlin, 1977, 123-130.

[40] М. Б. Гавриков, В. В. Савельев, "Равновесные конфигурации плазмы в приближении двухжидкостной магнитной гидродинамики с учетом инерции электронов", Тр. сем. им. И.Г. Петровского, 27:1 (2009), 3-66; англ. пер.: М. В. Gavrikov, V. V. Savelyev, "Equilibrium configurations of plasma in the approximation of two-fluid magnetohydrodynamics with electron inertia taken into account", J. Math. Sci. (New York), 163:1 (2009), 1-40.

[41] H. Helmholtz, Wissenschaftliche abhandlungen, J. A. Barth, Leipzig, 1895.

[42] А. Г. Столетов, Общедоступные лекиии и речи, М., 1902.

[43] G. Kirchhoff, "Zur Theorie freier Flüssigkeitsstrahlen", J. Reine Angew. Math. Grell. Berlin, 70 (1869), 289-298; см. также: Г. Р. Кирхгоф, Механика. Лекции по математической физике, Изд-во АН СССР, М., 1962.

[44] A. S. Demidov, "Equilibrium form of a steady plasma", Phys. Fluids, 21 (1978), 902-904.

[45] Г. М. Голузин, Геометрическая теория функиий комплексного переменного, 2-е изд., Наука, М., 1966.

[46] М.А. Лаврентьев, Б.В. Шабат, Методы теории функиий комплексного переменного, 4-е изд., Наука, М., 1973; 3-е изд., 1965.

[47] И. П. Натансон, Теория функиий вещественной переменной, 3-е изд., Наука, М., 1974; англ. пер. 1-го изд.: I.P. Natanson, Theory of functions of a real variable, Frederick Ungar, New York, 1955; Vol. II, 1961.

[48] А. Баджади, А.С. Демидов, “Теоремы существования, несуществования и регулярности в одной задаче со свободной границей”, Матем. сб., 122:1 (1983), 64-81; англ. пер.: A. Badzhadi, A. S. Demidov, "Existence, nonexistence and regularity theorems in a problem with a free boundary", Math. USSR-Sb., 50:1 (1985), $67-84$.

[49] Н. Е. Жуковский, “Видоизменение метода Кирхгофа для определения движения жидкости в двух измерениях при постоянной скорости, данной на неизвестной границе", Матем. сб., 15:1 (1890), 121-278; см. также Собр. соч., Т. II, ГИТТЛ, М.-Л., 1949.

[50] В.Н. Монахов, Краевые задачи со свободными границами для эллиптических систем уравнений, 2-е изд., Наука, Новосибирск, 1977; англ. пер.: V. N. Monakhov, Transl. Math. Monogr., 57 (1983).

[51] Л. Ниренберг, Лекции по нелинейному функииональному анализу, Мир, М., 1977; пер. с англ.: L. Nirenberg, Topics in nonlinear functional analysis, Courant Institute of Mathematical Sciences, New York University, New York, 1974.

[52] J. Leray, J. Schauder, "Topologie et équations fonctionnelles", Ann. Sci. École Norm. Sup. (3), 51 (1934), 45-78; pyc. пер.: ЖК. Лерэй, Ю. Шаудер, "Топология и функциональные уравнения", УМН, 1:3-4 (1946), 71-95.

[53] А. Картан, Элементарная теория аналитических функиий одного и нескольких комплексных переменных, ИЛ, М., 1963; пер. с фр.: Н. Cartan, Théorie élémentaire des fonctions analytiques d'une ou plusieurs variables complexes, Enseignement des Sciences, Hermann, Paris, 1961.

[54] Yong Liu, "The equilibrium plasma subject to skin effect", SIAM J. Math. Anal., 26:5 (1995), 1157-1183.

[55] В.И. Арнольд, Геометрические методы в теории обыкновенных дифференииалъных уравнений, 2-е изд., Удмурд. гос. ун-т, Ижевск, 2000. 
[56] А. С. Демидов, "Полная асимптотика решения задачи Дирихле для 2-мерного уравнения Лапласа с быстро осциллирующими граничными данными", Докл. PAH, 346:6 (1996), 732-734; англ. пер.: A. S. Demidov, "A complete asymptotics of the solution of the Dirichlet problem for a two-dimensional Laplace equation with rapidly oscillating boundary data", Dokl. Math., 53:1 (1996), 81-83.

[57] A. S. Demidov, V. V. Petrova, V. M. Silantiev, "On inverse and direct free boundary problems in the theory of plasma equilibrium in a Tokamak", C. R. Acad. Sci. Paris Sér. I Math., 323:4 (1996), 353-358.

[58] А. С. Демидов, А. А. Платущихин, "Явная формула для градиента гармонической функции по ее аналитическим данным Коши на аналитической кривой”, Матем. заметки, 87:1 (2010), 141-143.

[59] А. С. Демидов, А. С. Кочуров, А. Ю. Попов, "К задаче о реконструкции нелинейностей в уравнениях математической физики", Тр. сем. им. И.Г. Петровского, 27:1 (2009), 75-125; англ. пер.: A. S. Demidov, A. S. Kochurov, A. Yu. Popov, "То the problem of the recovery of non-linearities in equations of mathematical physics", J. Math. Sci., 163:1 (2009), 46-77.

[60] А. Д. Валиев, А. С. Демидов, "О неотрицательных тригонометрических полиномах с фиксированным средним, проходящих через заданные точки", Матем. заметки, 62:3 (1997), 468-471; англ. пер.: A. D. Valiev, A. S. Demidov, "Nonnegative trigonometric polynomials with fixed mean passing through given points", Math. Notes, 62:3 (1997), 390-392.

[61] Л. А. Галин, "Неустановившаяся фильтрация со свободной поверхностью”, Докл. AH CCCP, 47:4 (1945), 246-249.

[62] П. Я. Полубаринова-Кочина, "К вопросу о перемещении контура нефтеносности", Докл. АН СССР, 47:4 (1945), 254-257.

[63] П. Я. Полубаринова-Кочина, "О неустановившихся движениях в теории фильтрации: О перемещении контура нефтеносности", ПММ, 9:1 (1945), 79-90.

[64] П. П. Куфарев, "Решение задачи о контуре нефтеносности для круга", Докл. АН СCCP, 60:8 (1948), 1333-1334.

[65] Ю.П. Виноградов, П. П. Куфарев, “Об одной задаче фильтрации”, ПМM, 12:2 (1948), 181-198.

[66] Дж. Р. Окендон, С. Д. Ховисон, "Кочина и Хеле-Шоу в современной математике, естественных науках и технике", ПММ, 66:3 (2002), 515-524; англ пер.: J. R. Ockendon, S.D. Howison, "Kochina and Hele-Shaw in modern mathematics, natural science and industry", J. Appl. Math. Mech., 66:3 (2002), 505-512.

[67] П. Я. Кочина, Избранные труды. Гидродинамика и теория фильтрачии, Наука, M., 1991.

[68] B. Gustafsson, "Applications of variational inequalities to a moving boundary problem for Hele-Shaw flows", SIAM J. Math. Anal., 16:2 (1985), 279-300.

[69] B. Gustafsson, A. Vasil'ev, Conformal and potential analysis in Hele-Shaw cells, Adv. Math. Fluid Mech., Birkhäuser, Basel, 2006.

[70] Hele-Shaw flows and related problems: Papers from the conference held in Oxford (1998), European J. Appl. Math., 10, eds. S. D. Howison, J. R. Ockendon, Cambridge Univ. Press, New York, 1999.

[71] A. M. Meirmanov, B. Zaltzman, "Global in time solution to the Hele-Shaw problem with a change of topology", European J. Appl. Math., 13:4 (2002), 431-447.

[72] Л.Н. Александров, Кинетика кристаллизачии и перекристаллизации полупроводниковых пленок, Наука, Новосибирск, 1985.

[73] Е. Н. Каблов, Литые лопатки газотурбинных двигателей, МИСИС, М., 2001.

[74] B. G. Thomas, Ch. Beckermann (Eds.), Modeling of casting, welding advanced solidification processes (San Diego, CA, 1998), Minerals, Metals \& Materials Society, Warrendale, PA, 1998. 
[75] П. И. Плотников, В.Н.Старовойтов, "Задача Стефана как предел системы фазового поля", Дифб. уравнения, 29:3 (1993), 461-471; англ. пер.: P. I. Plotnikov, V. N. Starovoitov, "The Stefan problem with surface tension as a limit of the phase field model", Differential Equations, 29:3 (1993), 395-404.

[76] G. Caginalp, "Stefan and Hele-Shaw type problems as asymptotics limits of the phase-field equations", Phys. Rev. A (3), 39:11 (1989), 5887-5896.

[77] G. Caginalp, X. Chen, "Convergence of the phase field model to its sharp interface limits", European J. Appl. Math., 9:4 (1998), 417-445.

[78] В. Г. Данилов, Г. А. Омельянов, Е.В. Радкевич, "Асимптотическое решение системы фазового поля и модифицированная задача Стефана", Дифферени. уравнения, 31:3 (1995), 483-491; англ. пер.: V. G. Danilov, G. A. Omel'yanov, E. V. Radkevich, "Asymptotic behavior of the solution of a phase field system, and a modified Stefan problem", Differential Equations, 31:3 (1995), 446-454.

[79] J. Escher, G. Simonett, "Classical solutions of multidimensional Hele-Shaw models", SIAM J. Math. Anal., 28:5 (1997), 1028-1047.

[80] G. Prokert, "On evalution equations for moving domains", Z. Anal. Anwend., 18:1 (1999), 67-95.

[81] А. С. Демидов, “Эволюция возмущения окружности в задаче Стокса-Лейбензона для течения Хил-Шоу", Дифференииальные уравнения в частных производных, Современная математика и ее приложения, 2, 2003, 3-24; англ. пер.: A.S. Demidov, "Evolution of the perturbation of a circle in the Stokes-Leibenson problem for the Hele-Shaw flow", J. Math. Sci. (N. Y.), 123:5 (2004), 4381-4403.

[82] А. С. Демидов, "Эволюция возмущения окружности в задаче Стокса-Лейбензона для течения Хил-Шоу. II", Динамические системы и оптимизация, Современная математика и ее приложения, 24, 2005, 51-65; англ. пер.: А. S. Demidov, "Evolution of the perturbation of a circle in the Stokes-Leibenson problem for the Hele-Shaw flow. Part II", J. Math. Sci. (N. Y.), 139:6 (2006), 7064-7078.

[83] А. С. Демидов, "Об эволюции слабого возмущения окружности в задаче о течении Хил-Шоу", УМН, 57:6 (2002), 177-178; англ. пер.: А. S. Demidov, "On the evolution of a weak perturbation of a circle in the problem of a Hele-Shaw flow", Russian Math. Surveys, 57:6 (2002), 1212-1214.

[84] A. Antontsev, A. M. Meirmanov, V. Yurinsky, Hele-Shaw flow in two dimensions: Global-in-time classical solutions, Preprint № 6, Universidade da Beira Interior, Portugal, 1999.

[85] S. Richardson, "Hele-Shaw flows with a free boundary produced by the injection of fluid into a narrow channel", J. Fluid Mech., 56:4 (1972), 609-618.

[86] A.S. Demidov, J.-P. Lohéac, A quasi-contour model of Stokes-Leibenson problem for Hele-Shaw flows, CNRS UMR 5585 preprint 328, 2001.

[87] A.S. Demidov, J.-P. Lohéac, "On the evolution near some attractive manifold in a problem for the Hele-Shaw flows", Математические идеи П.Л. Чебышёва и их приложение к современным проблемам естествознания (Обнинск, 2002), Обнинский ин-т атомной энергетики, Обнинск, 2002, 37-38.

[88] A. S. Demidov, J.-P. Lohéac, "The Stokes-Leibenson problem for Hele-Shaw flows", Patterns and waves (St. Petersburg, 2002), eds. A. Abramian, S. Vaculenko, V. Volpert, AkademPrint, St. Petersburg, 2003, 103-124.

[89] О. А. Васильева, А. С. Демидов, "Конечноточечная модель задачи Стокса-Лейбензона для Хил-Шоу течения", Фундам. и прикл. матем., 5:1 (1999), 67-84.

[90] А.С. Демидов, "Полигональная модель для течения Хил-Шоу", УМН, 53:4 (1998), 195-196; англ. пер.: A.S. Demidov, "A polygonal model for Hele-Shaw flow", Russian Math. Surveys, 53:4 (1998), 846.

[91] А. С. Демидов, "О минимуме непрерывных функционалов от производных гармонической функции, параметризованной ее искомой линией уровня и/или другими граничными данными", Динамические системы и оптимизаиия, Современная математика и ее приложения, 24, Изд-во АН Грузии, Тбилиси, 2005, 
35-50; англ. пер.: A.S. Demidov, "On the minimum of continuous functionals of derivatives for a harmonic function parametrized by its desired level line and/or other boundary data", J. Math. Sci. (N. Y.), 139:6 (2006), 7047-7063.

[92] L. Föppl, "Wirbelbewegung hinter einem Kreiszylinder", Sitzb. Bayr. Akad. Wiss., 1913, 1-17.

[93] М. А. Лаврентьев, Б.В. Шабат, Проблемы гидродинамики и их математические модели, 2-е изд., Наука, М., 1977.

[94] А. С. Демидов, "Редукция к системе обыкновенных дифференциальных уравнений нестационарного гидродинамического уравнения Эйлера в плоской задаче кавитационного обтекания с граничным управлением", Матем. заметки, 86:1 (2009), 37-50; англ. пер.: A. S. Demidov, "Reduction of a nonstationary Euler hydrodynamic equation to a system of ordinary differential equations in the plane problem of cavitational flow with boundary control", Math. Notes, 86:1-2 (2009), $41-52$.

[95] В. И. Власов, "Вариация конформного отображения при сингулярном деформировании области и ее приложение к теории струй", Аналитические и численные методы решения задач математической физики, Изд-во ВЦ АН СССР, М., 1989, 41-48.

[96] A.S. Demidov, A.S. Kochurov, A. Yu. Protasov, "Optimization of the speed of motion for a flow by the Föppl-Lavrent'ev scheme", Russian J. Math. Phys. (в печати).

[97] Г. Биркгоф, Э. Сарантонелло, Струи, следы и каверны, Мир, М., 1964; пер. с англ.: G. Birkhoff, E. H. Zarantonello, Jets, wakes, and cavities, Academic Press, New York, 1957.

[98] Ф. Д. Гахов, Краевые задачи, 3-е изд., Наука, М., 1977; англ. пер. 1-го изд.: F. D. Gakhov, Boundary value problems, Pergamon Press, Oxford-New York-Paris; Addison-Wesley, Reading, MA-London, 1966.

[99] Г. Биркгоф, Гидродинамика: Методы, факты, подобие, 2-е изд., ИЛ, М., 1963; пер. с англ.: G. Birkhoff, Hydrodynamics. A study in logic, fact and similitude, Princeton Univ. Press, Princeton, NJ, 1960.

[100] М. И. Вишик, С. Л. Соболев, "Общая постановка некоторых краевых задач для эллиптических дифференциальных уравнений в частных производных", Докл. AH CCCP, 111:3 (1956), 521-523.

[101] J.-L. Lions, E. Magenes, Problèmes aux limites non homogènes et applications, Vol. I, Travaux et Recherches Mathématiques, 17, Dunod, Paris, 1968; Vol. II, Travaux et Recherches Mathématiques, 18, 1968; Vol. III, Travaux et Recherches Mathématiques, 20, 1970; рус. пер.: Дж.-Л. Лионс, Э. Мадженес, Неоднородные граничные задачи и их приложения, т. I, Мир, М., 1971.

[102] Э. Мадженес, "Интерполяционные пространства и уравнения в частных производных", УМН, 21:2 (1966), 169-218.

[103] Р. Курант, Д. Гильберт, Уравнения с частными производными, Мир, М., 1964; пер. с англ.: R. Courant, D. Hilbert, Methods of mathematical physics. Vol. II: Partial differential equations, Interscience Publishers, New York-London, 1962.

[104] O.A. Oleinik, R. Temam, G. A. Yosifian, "Some nonlinear homogenization problems", Appl. Anal., 57:1-2 (1995), 101-118.

[105] R. Aymar, P. Barabaschi, Y. Shimomura, "The ITER design", Plasma Phys. Control. Fusion, 44 (2002), 519-565.

[106] T. C. Luce, "Development of steady-state advanced tokamak research in the DIII-D tokamak", Fusion Science and Technology, 48:2 (2005), 1212-1225.

[107] К. В. Брушлинский, В. В. Савельев, "Магнитные ловушки для удержания плазмы", Матем. моделирование, 11:5 (1999), 3-36.

[108] О.И. Богоявленский, "Точные глобальные равновесия плазмы”, УМH, 55:3 (2000), 63-102; англ. пер.: O. I. Bogoyavlenskij, "Exact global plasma equilibria", Russian Math. Surveys, 55:3 (2000), 463-500. 
[109] В. Л. Поляченко, А. М. Фридман, Равновесие и устойчивость гравитирующих систем, Наука, М., 1976.

[110] В. Д. Шафранов, "О магнитогидродинамических равновесных конфигурациях", ЖЭЭТФ, 33:3(9) (1957), 710-722; англ. пер.: V. D. Shafranov, "On magnetohydrodynamical equilibrium configurations", Soviet Physics JETP, 6 (1958), 545-554.

[111] R. Lüst, A. Schlüter, "Axialsymmetrische magnetohydrodynamische gleichgewichtskonfigurationen", Z. Naturf., 12A (1957), 850.

[112] H. Grad, H. Rubin, "Hydromagnetic equilibria and force-free fields", Proceedings of the 2nd United Nations International Conference on the Peaceful Uses of Atomic Energy (Geneva, 1958), Columbia Univ. Press, New York, 1958.

[113] М.И. Гуревич, "Теория струй идеальной жидкости”, Отрывные и кавитационные течения, 2-е изд., Наука, М., 1979; англ. пер. 1-го изд.: М. I. Gurevich, The theory of jets in an ideal fluid, Internat. Ser. Monogr. Pure Appl. Math., 93, Pergamon Press, Oxford-New York-Toronto, 1966.

[114] Н.Е. Кочин, И. А. Кибель, Н. В. Розе, Теоретическая гидромеханика, 6-е изд. (в двух частях), Физматгиз, М., 1963; англ. пер.: N.E. Kochin, I. A. Kibel', N. V. Rose, Theoretical hydromechanics, Wiley, New York-London-Sydney, 1964.

[115] M. Planck, "Zur Theorie der Flüssigkeitsstrahlen", Annalen der Physik und Chemie (Wiedemanns Ann.), ser. 2, XXI (1884), 499-509.

[116] С. А. Чаплыгин, "О некоторых случаях движения твердого тела в жидкости”, Матем. сб., XX (1897), 114-170, 173-246.

[117] T. Levi-Civita, "Scie e leggi di resistenzia", Rend. Circolo Math. Palermo, 23:1 (1907), 1-37.

[118] A.S. Demidov, "Some applications of the Helmholtz-Kirchhoff method (equilibrium plasma in tokamaks, Hele-Shaw flow, and high-frequency asymptotics)", Russian J. Math. Phys., 7:2 (2000), 166-186.

[119] B. W. Thompson, "Secondary flow in a Hele-Shaw cell", J. Fluid Mech., 31:2 (1968), 379-395.

[120] J. R. Ockendon, "Linear and nonlinear stability of a class of moving boundary problems", Free boundary problems, Vol. II (Pavia, 1979), Ist. Naz. Alta Mat. Francesco Severi, Rome, 1980, 443-478.

[121] А. С. Демидов, Ж. Мвамбакана, И. А. Федотов, "Альтернирующий метод Шварца для эллиптических краевых задач, удовлетворяющих принципу максимума”, Матем. заметки, 78:4 (2005), 622-624; англ. пер.: A.S. Demidov, J. Mwambakana, I. A. Fedotov, "Schwartz alternating method for elliptic boundary-value problems satisfying the maximum principle", Math. Notes, 78:3-4 (2005), 577-580.

[122] К. Иосида, Функционалъный анализ, Мир, М., 1967; пер. с англ.: K. Yosida, Functional analysis, Grundlehren Math. Wiss., 123, Academic Press, New York; Springer, Berlin, 1965.

[123] В.И. Арнольд, "И. Г. Петровский, топологические проблемы Гильберта и современная математика", УМH, 57:4 (2002), 197-207; англ. пер.: V. I. Arnol'd, "I. G. Petrovskii, Hilbert's topological problems, and modern mathematics", Russian Math. Surveys, 57:4 (2002), 833-845.

[124] J. Leray, J. L. Lions, "Quelques résultats de Višik sur les problèmes elliptiques non linéaires par les méthodes de Minty-Browder", Bull. Soc. Math. France, 93 (1965), 97-107.

А. C. Демидов (A.S. Demidov)

Московский государственный университет

им. М. В. Ломоносова;

Московский физико-технический институт

(государственный университет)

E-mail: alexandre.demidov@mtu-net.ru
Поступила в редакцию 11.12.2009 